Aus der Poliklinik für Präventive Zahnmedizin, Parodontologie und Kariologie (Prof. Dr. med. dent. A. Wiegand) im Zentrum Zahn-, Mund- und Kieferheilkunde der Medizinischen Fakultät der Universität Göttingen

\title{
Präparation gekrümmter Wurzelkanäle mit drei Nickel-Titan-Systemen - eine Mikro-CT- Studie
}

\author{
INAUGURAL-DISSERTATION \\ zur Erlangung des Doktorgrades \\ der Zahnmedizin \\ der Medizinischen Fakultät der \\ Georg-August-Universität zu Göttingen
}

vorgelegt von

Jonas Robert Wilhelm Pult

aus

Braunschweig

Göttingen 2020 


\section{Betreuungsausschuss}

Betreuer/in:

Prof. Dr. med. dent. M. Hülsmann

Ko-Betreuer/in:

Prof. Dr. med. dent. M. Rödiger

\section{Prüfungskommission}

Referent/in:

Prof. Dr. med. dent. M. Hülsmann

Ko-Referent/in:

Prof. Dr. med. dent. M. Rödiger

Drittreferent/in:

Prof. Dr. med. M. Schön

Datum der mündlichen Prüfung: 24.03.2021 
Hiermit erkläre ich, die Dissertation mit dem Titel "Präparation gekrümmter Wurzelkanäle mit drei Nickel-TitanSystemen - eine Mikro-CT-Studie" eigenständig angefertigt und keine anderen als die von mir angegebenen Quellen und Hilfsmittel verwendet zu haben.

Braunschweig, den .............. 
Die Daten, auf denen die vorliegende Arbeit basiert, wurden teilweise publiziert:

Haupt F, Pult JRW, Hülsmann M (2020): Micro-computed tomographic evaluation of the shaping ability of 3 reciprocating single-file nickel-titanium systems on single- and double-curved root canals. J Endod $\underline{46}, 1130-1135$ 


\section{Inhaltsverzeichnis}

Abbildungsverzeichnis ........................................................... III

Tabellenverzeichnis ........................................................... IV

Abkürzungsverzeichnis ..........................................................

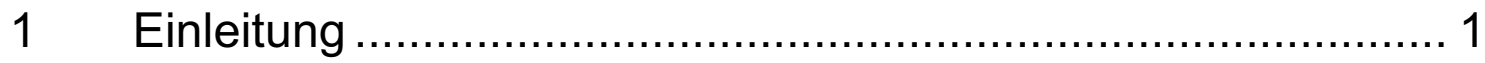

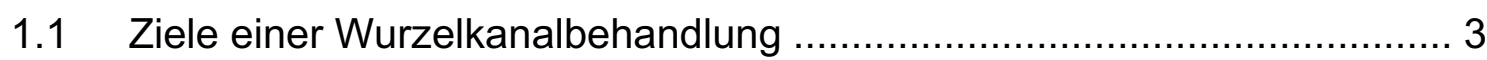

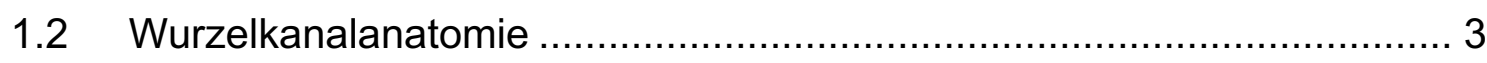

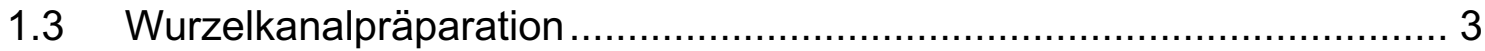

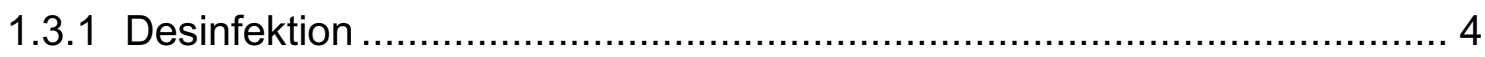

1.3.2 Präparationsregeln ................................................................. 4

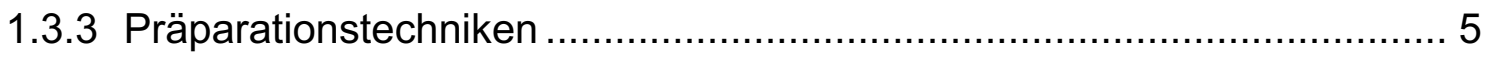

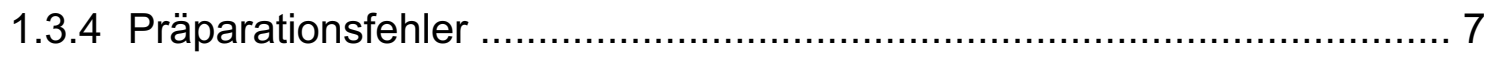

1.4 Maschinelle Wurzelkanalpräparation mit Nickel-Titan-Instrumenten ...... 10

1.4.1 Rotierend arbeitende Nickel-Titan-Instrumente .................................. 11

1.4.2 Reziprok arbeitende Nickel-Titan-Instrumente ….............................. 11

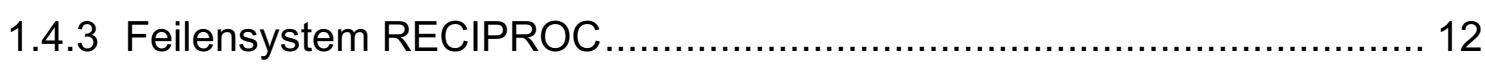

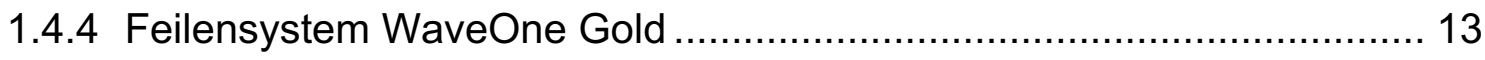

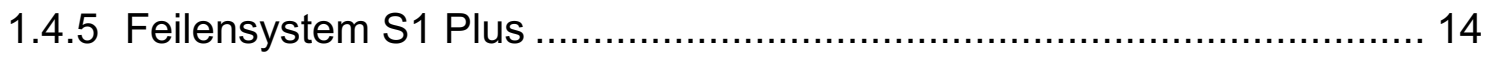

1.5 Mikro-Computertomographie (Mikro-CT) .......................................... 15

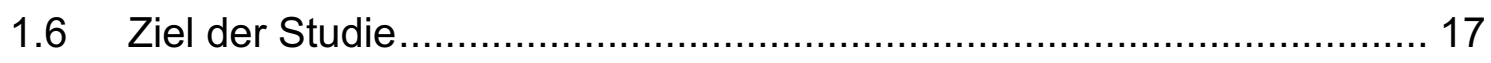

$2 \quad$ Material und Methoden .................................................... 18

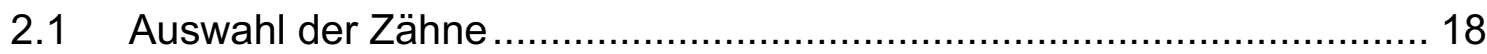

2.2 Einteilung der Versuchsgruppen.................................................... 22

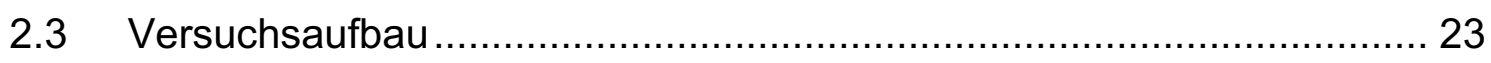

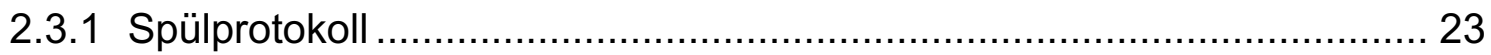

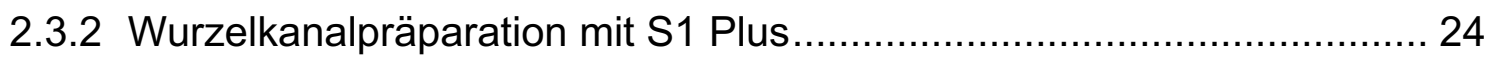

2.3.3 Wurzelkanalpräparation mit WaveOne Gold ...................................... 24

2.3.4 Wurzelkanalpräparation mit RECIPROC …….................................. 24

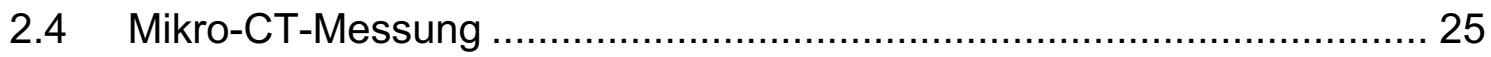

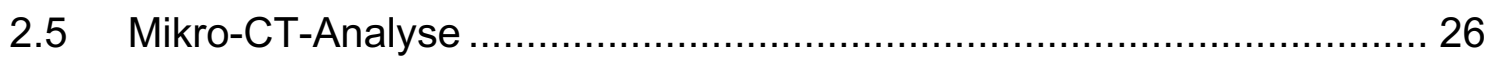




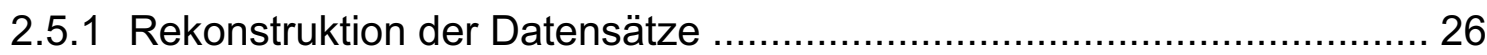

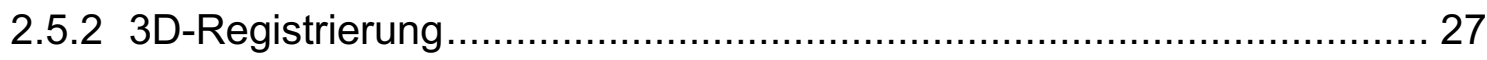

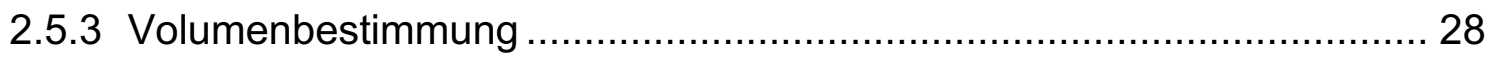

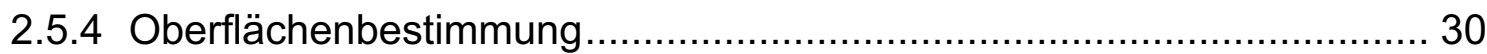

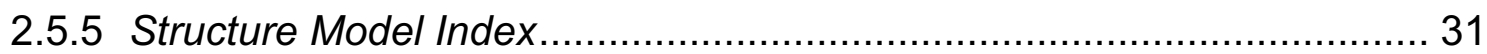

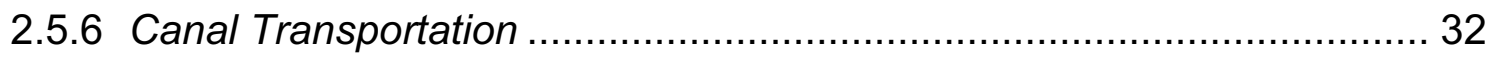

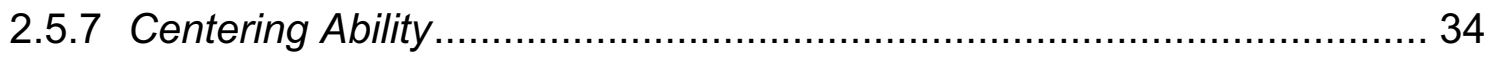

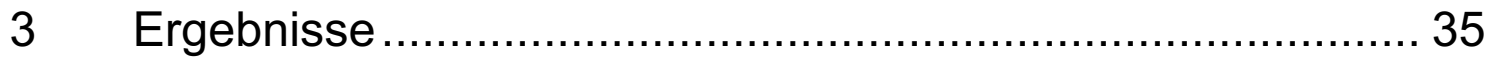

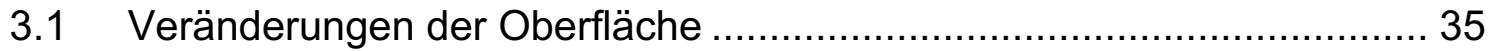

3.1.1 Anteil der unpräparierten Oberfläche.............................................. 36

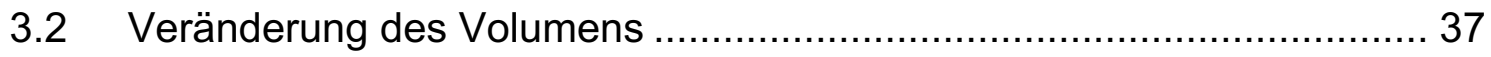

3.3 Veränderung des Structure Model Index …….................................... 39

3.4 Veränderung der Canal Transportation ............................................. 41

3.5 Veränderung der Centering Ability ................................................... 44

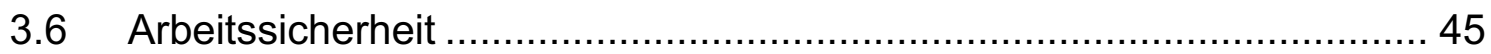

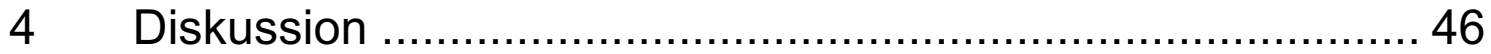

4.1 Diskussion der Methode ................................................................. 46

4.1.1 Auswahl der Versuchszähne .......................................................... 46

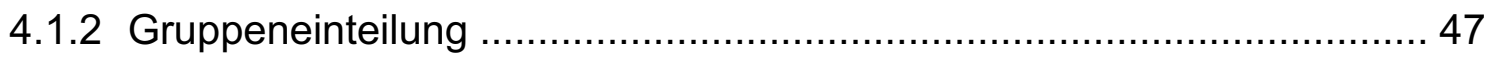

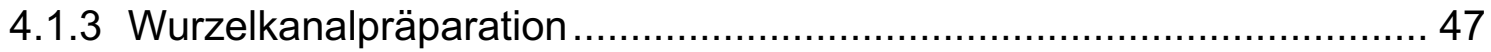

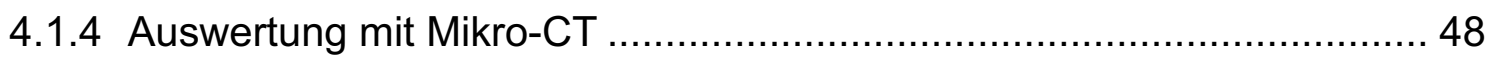

4.1.5 Berechnung der Versuchsparameter ............................................. 50

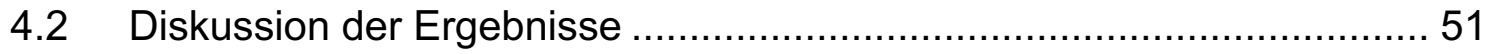

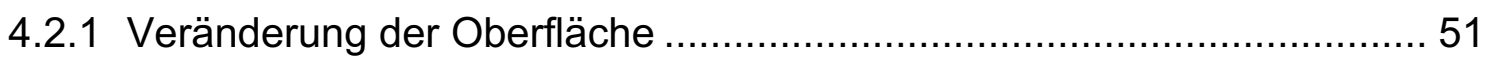

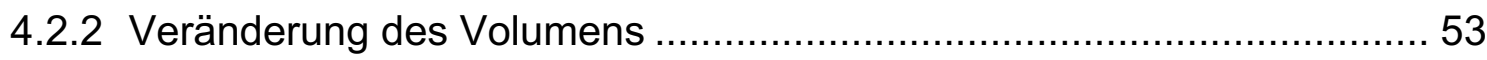

4.2.3 Veränderung des Structure Model Index ............................................. 54

4.2.4 Veränderung von Canal Transportation und Centering Ability ............... 55

$5 \quad$ Zusammenfassung .............................................................. 58

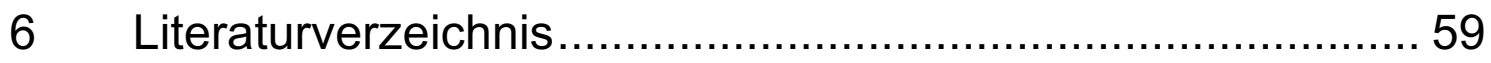




\section{Abbildungsverzeichnis}

Abb. 1: Radiologische Darstellung einer präparationsbedingten Bildung von zip und elbow.

Abb. 2: Radiologische Darstellung einer präparationsbedingten Stufenbildung im apikalen Wurzelkanaldrittel

Abb. 3: Radiologische Darstellung einer präparationsbedingten apikalen Perforation

Abb. 4: Radiologische Darstellung einer präparationsbedingten lateralen Perforation

Abb. 5: Feile R25 (25/.08) des Systems RECIPROC

Abb. 6: Feile Primary (25/.07) des Systems WaveOne Gold

Abb. 7: Feile Standard (25/.06) des Systems S1 Plus 15

Abb. 8: Schematische Darstellung eines Mikro-CT 16

Abb. 9: Röntgenmessaufnahme eines mesiobukkalen Wurzelkanals 19

Abb. 10: Röntgenmessaufnahme eines mesiolingualen Wurzelkanals . 20

Abb. 11: Messung des Krümmungswinkels nach Schneider (1971). 21

Abb. 12: Berechnung des Krümmungsradius nach Schäfer et al. (2002). 21

Abb. 13: Berechnung einer zweiten Wurzelkanalkrümmung nach Cunningham und Senia (1992)

Abb. 14: Versuchszahn im nicht röntgenopaken Messzylinder 25

Abb. 15: Mikro-CT Bruker SkyScan 1272. 26

Abb. 16: 3D-Darstellung eines Versuchszahns in mesio-distaler Ansicht 29

Abb. 17: 3D-Darstellung eines Versuchszahns mit s-shape-Wurzelkanalverlauf in koronal-apikaler Ansicht.

Abb. 18: 3D-Darstellung eines Versuchszahns mit s-shape-Wurzelkanalverlauf in bucco-lingualer Ansicht. 30

Abb. 19: Wurzelkanalquerschnitte bei $1 \mathrm{~mm}, 3 \mathrm{~mm}, 5 \mathrm{~mm}, 7 \mathrm{~mm}$ vom Apex entfernt. 33

Abb. 20: Messung der Canal Transportation nach Gambill et al. (1996) ... 33

Abb. 21: Veränderung der Wurzelkanaloberfläche 37

Abb. 22: Anteil der unpräparierten Wurzelkanaloberfläche

Abb. 23: Volumenveränderung der Wurzelkanäle in 38

Abb. 24: Veränderung des SMI. 


\section{Tabellenverzeichnis}

Tab. 1: Mittelwerte der Krümmungswinkel und Krümmmungsradien der mesiobukkalen Wurzelkanäle

Tab. 2: Mittelwerte der Krümmungswinkel und Krümmmungsradien der mesiolingualen Wurzelkanäle

Tab. 3: Veränderungen der Wurzelkanaloberfläche und Anteil der unpräparierten Wurzelkanaloberfläche.

Tab. 4: Veränderungen des Wurzelkanalvolumens ................................. 38

Tab. 5: Veränderungen des Structure Model Index ..................................... 39

Tab. 6: $p$-Werte der zweifaktoriellen ANOVA....................................... 40

Tab. 7: $p$-Werte der dreifaktoriellen ANOVA....................................... 41

Tab. 8: p-Werte des Tukey-Tests zwischen den Feilengruppen...................... 42

Tab. 9: p-Werte des Scheffé-Tests ohne Abhängigkeit von der Feilengruppen in allen Querschnitten

Tab. 10: p-Werte des Scheffé-Tests innerhalb der Gruppe S1 Plus in allen Querschnitten

Tab. 11: p-Werte des Scheffé-Tests innerhalb der Gruppe WOG in allen Querschnitten

Tab. 12: p-Werte des Scheffé-Tests innerhalb der Gruppe RECIPROC in allen Querschnitten.

Tab. 13: Mittelwerte der Canal Transportation ohne Berücksichtigung der Mesial- oder Distalverlagerung

Tab. 14: Mittelwerte der Canal Transportation mit Berücksichtigung der Mesialund Distalverlagerung

Tab. 15: Mittelwerte der Centering Ability mit Berücksichtigung der Mesial- und Distalverlagerung 


\section{Abkürzungsverzeichnis}

CA

CT

E-Modul

Mikro-CT

$\mathrm{NiTi}$

$\mathrm{ROI}$

R-Phase

SMI

WOG
Centering Ability

Canal Transportation

Elastizitätsmodul

Mikro-Computertomographie

Nickel-Titan

region of interest

rhomboedrische Übergangsphase

Structure Model Index

WaveOne Gold 


\section{$1 \quad$ Einleitung}

Die moderne Zahnmedizin trägt maßgeblich zum langfristigen Zahnerhalt bei. Die Endodontie als großes Teilgebiet der konservierenden Zahnmedizin befasst sich dabei mit dem Pulpagewebe und dessen Form, Funktion und Gesundheit. Insbesondere stehen die Diagnostik von Verletzungen und endodontischen Erkrankungen sowie deren Prävention und Therapie im Mittelpunkt (European Society of Endodontology 2006). Der Erfolg endodontischer Behandlungen wird verschiedenen Studien zufolge mit bis zu 90 \% angegeben (Eyuboglu et al. 2017; Farzaneh et al. 2004).

Die Wurzelkanalpräparation hat sich mit Beginn der Verwendung von Feilen aus Nickel-Titan-Legierungen (NiTi-Legierungen) zur maschinellen Wurzelkanalpräparation deutlich weiterentwickelt. Walia et al. (1988) verwendeten als Erste die neuartige Legierung in der Endodontie. Die Legierung zeichnet sich vor allem durch eine erhöhte Flexibilität und ein erhöhtes Rückstellvermögen der Instrumente nach erfolgter elastischer Verformung ohne erkennbare plastische Deformation aus (Hülsmann et al. 2005; Viana et al. 2010; Zinelis et al. 2010). Somit können komplexere Wurzelkanalgeometrien präziser bearbeitet werden.

Seitens der Krankenkassen wird auf eine bessere Wirtschaftlichkeit der Zahnbehandlung geachtet. Eine Wurzelkanalpräparation sollte qualitativ hochwertig, aber auch so schnell wie möglich mit möglichst geringem Materialaufwand erfolgen. Die Verwendung von NiTi-Systemen, bestehend aus nur einer Präparationsfeile, soll neben der zeitlichen Ersparnis auch eine Vereinfachung des Arbeitsablaufes und eine Kostenersparnis bewirken.

Seit 2008 werden, basierend auf einer Studie von Yared (2008), reziprok arbeitende Feilensysteme zur Wurzelkanalpräparation verwendet. Dabei bewegt sich die Feile nicht mehr in einer Rotation von 360 Grad, sondern vollführt eine Teilrotation meist entgegen dem Uhrzeigersinn, was von einer gegenläufigen Bewegung abgelöst wird. Die ursprüngliche Idee der reziproken Arbeitsweise wurde bereits 1964 mit der Vorstellung des Giromatic-Winkelstücks (MicroMéga, Besançon, Frankreich) entwickelt und nun wieder aufgegriffen (Hülsmann et al. 2005).

Im Fokus dieser Studie stehen drei NiTi-Einfeilensysteme in reziproker Arbeitsweise: S1 Plus (Sendoline, Täby, Schweden), WaveOne Gold (Dentsply Sirona, Ballaigues, Schweiz) und RECIPROC (VDW, München). 
Es sollen Veränderungen im Wurzelkanalvolumen, der Wurzelkanaloberfläche und mögliche Wurzelkanalbegradigungen untersucht werden, um eine Bewertung der Praxistauglichkeit der drei Feilensysteme zu ermöglichen. 


\section{$1.1 \quad$ Ziele einer Wurzelkanalbehandlung}

Durch eine Wurzelkanalbehandlung sollen ein langfristiger Erhalt eines pulpaerkrankten Zahnes als funktionsfähige Kaueinheit erreicht und zudem die Gesundheit entzündlich veränderter periradikulärer Strukturen wiederhergestellt werden (European Society of Endodontology 2006). Bei klinischer Symptomfreiheit und radiologisch unauffälligem Parodontalspalt kann von apikaler Gesundheit und somit einem Therapieerfolg gesprochen werden (DGZMK 2000).

\subsection{Wurzelkanalanatomie}

Im Verlauf des Wurzelkanals treten bei nahezu allen Zähnen Krümmungen auf, die über den ganzen Wurzelkanal oder lediglich im apikalen Bereich verlaufen können (Cunningham und Senia 1992; Vertucci 2005). Apikale Krümmungen treten in etwa $85 \%$ aller Wurzelkanäle auf (Pineda und Kuttler 1972). Die Krümmungen können dreidimensional in jeder Richtung vorliegen. Doppelte Wurzelkanalkrümmungen, auch als s-shaped bezeichnet, können ebenfalls in allen Richtungen des dreidimensionalen Wurzelkanals auftreten und sind in bis zu $30 \%$ aller Unterkiefermolaren vorhanden (Cunningham und Senia 1992).

Erste Unterkiefermolaren, welche in der vorliegenden Studie verwendet werden, besitzen meist zwei gut ausgeprägte Wurzeln, bei der die mesiale Wurzel oft eine deutliche Krümmung aufweist und häufig einen mesiobukkalen und mesiolingualen Kanal besitzt (de Pablo et al. 2010). Ein dritter, mittlerer mesialer Wurzelkanal weist lediglich eine Inzidenz von 2,6 \% auf (de Pablo et al. 2010). Die distale Wurzel hat meist einen geraden Verlauf, entweder mit einem weiten ovalen Wurzelkanal oder mit zwei runden Wurzelkanälen (Skidmore und Bjorndal 1971).

\subsection{Wurzelkanalpräparation}

Die Präparation des Wurzelkanals basiert auf einer kombinierten chemomechanischen Erweiterung und Reinigung mit anschließender Obturation des endodontischen Hohlraumsystems (Hülsmann 2013; Hülsmann et al. 2005; Schäfer und Zapke 2000). Die Ziele der Wurzelkanalpräparation liegen in der vollständigen Entfernung des infizierten Pulpagewebes und Dentins und der drastischen Reduktion der Keimzahl im Endodont. Ein Wieder- oder Neueintritt von Keimen ist dabei zu vermeiden (Dittmer 2003; Heuer 1963; Meyer 1991; Rödig 2009; Schilder 1974). 
Eine ausreichende Präparation des Wurzelkanalsystems muss gewährleistet sein, um eine suffiziente Versiegelung mit Wurzelfüllmaterial zu gewährleisten. Die Präparation soll dabei so viel Zahnhartsubstanz wie möglich erhalten. (European Society of Endodontology 2006; Hülsmann 2008; Rödig 2009).

\subsubsection{Desinfektion}

Bei alleiniger mechanischer Wurzelkanalpräparation kann eine vollständige Reinigung des Wurzelkanalsystems aufgrund der komplexen Wurzelkanalanatomie nicht erreicht und eine Bakterienfreiheit somit nicht erzielt werden (Ørstavik et al. 1991; Rödig 2009). Etwa ein Drittel der kompletten Wurzelkanaloberfläche bleibt bei der Wurzelkanalpräparation unbearbeitet (Hülsmann 2006).

Durch die Präparation angefallenes Spanmaterial kann ohne Spülung des Wurzelkanals nicht entfernt werden (Shen et al. 2013). Während der Präparation bildet sich zudem eine Schmierschicht auf der Dentinoberfläche (smear layer), die durch eine weitere Präparation der Wurzelkanalwand ebenfalls nicht entfernt werden kann (Hülsmann 2008).

Zur Verbesserung der Reinigungswirkung des Wurzelkanalsystems können chemische Spülflüssigkeiten zur Entfernung der smear layer und Spülung der nicht präparierten Wurzelanalanteile eingesetzt werden (Hülsmann 2013).

Durch die Kombination aus Wurzelkanalpräparation und korrekter Wurzelkanalspülung kann eine Entfernung von 90 \% aller Keime aus dem Wurzelkanal erreicht werden (Siqueira et al. 1999).

\subsubsection{Präparationsregeln}

Ruddle (2002) und Schilder (1974) betonten, dass die Wurzelkanalpräparation einer der wichtigsten Schritte bei der Wurzelkanalbehandlung sei. Daher besitzen die von Schilder (1974) aufgestellten Präparationsregeln heute weiterhin Gültigkeit:

Die Verbreiterung des Wurzelkanals von apikal nach koronal sollte gleichmäßig in einem continuous taper erfolgen. Die Präparation folgt dabei stets entlang der ursprünglichen Anatomie des Wurzelkanals. Der Wurzelkanalquerschnitt ist koronal am Größten und sollte an jedem apikal gelegeneren Punkt kleiner werden. Die Gestaltung des Foramen apicale ist so klein umzusetzen, wie es praktisch möglich ist. Präparationsbedingte Verlagerungen des Foramen apicale sind dabei zu vermeiden. 
Über die von Schilder aufgestellten Regeln hinaus sollte ein ausreichender Dentinabtrag erfolgen, da die Penetrationstiefe von Mikroorganismen entlang der Dentintubuli bis zu $300 \mu \mathrm{m}$ betragen kann (Siqueira et al. 2002).

Eine Überinstrumentierung oder Unterinstrumentierung des Wurzelkanals sind zudem zu vermeiden, sodass die nach der Röntgenmessaufnahme festgelegte Arbeitslänge auch nach abgeschlossener Präparation eingehalten wird (Rödig 2009).

\subsubsection{Präparationstechniken}

Mithilfe unterschiedlicher Techniken kann eine Präparation nach den in Kapitel 1.3.2 beschriebenen Vorgaben erreicht werden. Die meisten Techniken verwenden zur Präparation eine Abfolge verschiedener Instrumente mit unterschiedlicher Größe und Konizität. Dabei erfolgt die Instrumentierung des Wurzelkanals entweder von koronal nach apikal oder von apikal nach koronal. Die am Häufigsten verwendeten manuellen Techniken zur Präparation von Wurzelkanälen werden im Folgenden erläutert:

\subsubsection{Standardized endodontic technique}

Bei der von Ingle (1961) vorgestellten Technik sollen alle verwendeten Wurzelkanalfeilen auf die vollständige vorab festgelegte Arbeitslänge eingebracht werden. Die endgültige Konizität und Größe des Wurzelkanals entspricht somit Form und Größe der zuletzt verwendeten Wurzelkanalfeile (Hülsmann et al. 2005). Hieraus resultieren vor allem Wurzelkanalpräparationen mit sehr geringen Konizitäten (Hülsmann 2008).

\subsubsection{Step-back technique}

Die step-back technique gilt als weiterentwickelte standardized endodontic technique, bei der zunächst der Wurzelkanal auf voller Arbeitslänge um mehrere ISOGrößen erweitert wird. Anschließend wird in apikal-koronaler Richtung eine sukzessive Stufenpräparation begonnen, bei der jede größere Wurzelkanalfeile mit einer Reduktion der Arbeitslänge um einen Millimeter verwendet wird (Clem 1969; Hülsmann 2008). Eine deutlich höhere Konizität wird erzielt und das Risiko von Wurzelkanalbegradigungen oder Perforationen verringert (Rödig 2009). 


\subsubsection{Crown-down technique}

Im Gegensatz zur step-back technique wird bei der crown-down technique zunächst eine koronale Erweiterung des Wurzelkanals bis zum Beginn der Wurzelkanalkrümmung mit hoher Konizität geschaffen, ohne ein Wurzelkanalinstrument auf Arbeitslänge einzubringen. Anschließend wird der apikale Bereich des Wurzelkanals auf voller Arbeitslänge mit kleineren Konizitäten bis zur gewünschten Größe erweitert (Goerig et al. 1982; Hülsmann et al. 2005). Durch die deutliche koronale Verbreiterung hat die Wurzelkanalfeile weniger Friktion bei weniger Wurzelkanalwandkontakt und kann apikale Wurzelkanalbereiche besser präparieren. Außerdem soll durch die Präparation die Menge des nach periapikal extrudierten Debris verringert werden, was im Vergleich zur step-back technique nachgewiesen werden konnte (Al-Omari und Dummer 1995; Ferraz et al. 2001; RuizHubard et al. 1987).

\subsubsection{Balanced-force technique}

Die von Roane et al. (1985) entwickelte Technik verwendet spezielle „Flex-RFeilen“, welche eine nicht schneidende Spitze besitzen. Das Vorgehen basiert auf einer crown-down technique (Hülsmann et al. 2005).

Die Feilen werden mit einer rotierenden Bewegung im Uhrzeigersinn von maximal $180^{\circ}$ in den Wurzelkanal eingebracht und anschließend in die Gegenrichtung um maximal $120^{\circ}$ mit apikalem Druck rotiert. Abschließend wird das Instrument wieder im Uhrzeigersinn aus dem Wurzelkanal rotiert (Hülsmann et al. 2005).

Mit dieser Technik lassen sich vor allem apikal gekrümmte Wurzelkanäle gut bearbeiten, da das Instrument nicht auf der gesamten Länge schneidet und eine gute Centering Ability (CA) aufgrund der nicht schneidenden Spitze aufweist. Ein Vorbiegen der Feilen ist nicht notwendig (Hülsmann et al. 2005; Ruddle 2002). Im Vergleich zu der stepback- oder standardized technique ist eine deutlich geringere Wurzelkanalbegradigung feststellbar (Backman et al. 1992; Calhoun und Montgomery 1988).

\subsubsection{Single-file technique}

Die Technik basiert auf der Verwendung von nur einer Wurzelkanalfeile, welche in maschinell reziprok bewegender Arbeitsweise eingesetzt wird (Yared 2008). Die verwendeten Feilen bestehen aus NiTi-Legierungen und besitzen am Ende des Arbeitsteils größere Konizitäten. Die Anwendung basiert auf der crown-down 
technique, bei der mit derselben Feile ohne Druck die kontinuierliche apikale Präparation mit regelmäßiger Spülung durchgeführt wird. Die Präparation ist abgeschlossen, wenn die Arbeitslänge erreicht ist. Der Vorteil dieser Technik liegt neben Zeitersparnis und Materialkosteneinsparung in der Reduzierung der Gefahr einer Kreuzkontamination bei mehrfacher Verwendung der Instrumente (Ruddle 2012; Yared 2008). Im Vergleich zu Mehrfeilensystemen können bei der singlefile technique eine gleichwertige Reinigungswirkung und Präparationsform erzielt werden (Bach und Hülsmann 2019).

Die in dieser Studie verwendeten Feilensysteme werden in der single-file technique eingesetzt.

\subsubsection{Präparationsfehler}

Gefördert durch die komplexe dreidimensionale Wurzelkanalanatomie entstehen leicht präparationsbedingte Fehler und Komplikationen. Vor allem gekrümmte Wurzelkanäle können bei der Präparation mit Stahlinstrumenten Fehler provozieren (Glickmann und Dumsha 1997; Weine et al. 1976; Weine et al. 1975):

Die Wurzelkanalfeile zeigt im Wurzelkanal die Tendenz sich zu begradigen (Pettiette et al. 1999). Dadurch wird koronal vermehrt die innere Krümmung des Wurzelkanals präpariert und apikal eine Verbeiterung des Wurzelkanals zur äußeren Krümmung erreicht. Die resultierende Kanalform im apikalen Bereich wird als zip (Trichter) bezeichnet und in Abb. 1 dargestellt (Hülsmann et al. 2005; Rödig 2009). Einhergehend mit einem zip entsteht am Punkt der größten Krümmung eine Einziehung, welche elbow (Isthmus) genannt wird (Abb. 1). Die Reinigung und spätere Obturation des Wurzelkanals kann an diesem Punkt erschwert werden (Hülsmann et al. 2005).

Bei der Verwendung von Stahlinstrumenten mit schneidender Spitze kann bei Rotationsbewegungen eine Stufe an der äußeren Krümmung präpariert werden, die nur schwer zu überwinden ist und häufig zu Blockaden führt (Abb. 2). Die Stufenbildung und Blockaden können in einem Verlust an Arbeitslänge resultieren (Hülsmann et al. 2005). Die Stufenbildung ist abhängig von der Wurzelkanalkrümmung und der verwendeten Wurzelkanalfeile (Greene und Krell 1990; Kapalas und Lambrianidis 2000). Stufenbildungen können durch das Vorbiegen der Wurzelkanalfeile vermieden werden (Linßen 2013).

Als weiterführende Komplikation kann bei bereits vorhandener Stufe im Wurzelkanal und fortgeführter Präparation eine Perforation des Wurzelkanals entstehen (Abb. 3). Neben der klinischen Beschwerdesymptomatik bleibt der apikal der Perforation liegende Abschnitt des Wurzelkanals unpräpariert (Hülsmann et al. 
2005). Bei Überinstrumentierung und Wurzelkanalbegradigung in gekrümmten Wurzelkanälen kann an der inneren Krümmung des Wurzelkanals ebenfalls eine Perforation des Wurzelkanals entstehen (Abb. 4). Diese wird als laterale Perforation oder strip perforation bezeichnet (Hülsmann et al. 2005).

Die Fraktur einer Wurzelkanalfeile ist ein weiterer iatrogener Präparationsfehler. Ein Torsionsbruch entsteht, wenn eine Feile im Wurzelkanal eingeklemmt wird und anschließend ein weiteres Drehmoment auf die Feile einwirkt. Die Feile bricht, wenn die Elastizitätsgrenze der verwendeten Legierung überschritten wird (Cheung 2009; Eggert und Gernhardt 2012).

Ermüdungsbrüche entstehen bei mikroskopischen und makroskopischen Gefügeveränderungen der Legierung mit Rissbildung bei häufiger Anwendung und Belastung der Wurzelkanalfeile (Cheung 2009). Die Häufigkeit von intrakanalären Instrumentenfrakturen bei der Präparation mit Handfeilen liegt zwischen $0,25 \%$ und $6 \%$ (Crump und Natkin 1970; Hülsmann und Schinkel 1999; Iqbal et al. 2006; Spili et al. 2005; Tzanetakis et al. 2008; Ungerechts et al. 2014). Bei der maschinellen Anwendung von Nickel-Titan-Instrumenten liegt die Häufigkeit intrakanalärer Instrumentenfrakturen bei 0,4 \% - 3,7 \% (Al-Fouzan 2003; Parashos und Messer 2006; Ramirez-Salomon et al. 1997; Schäfer et al. 2004; Spili et al. 2005). 


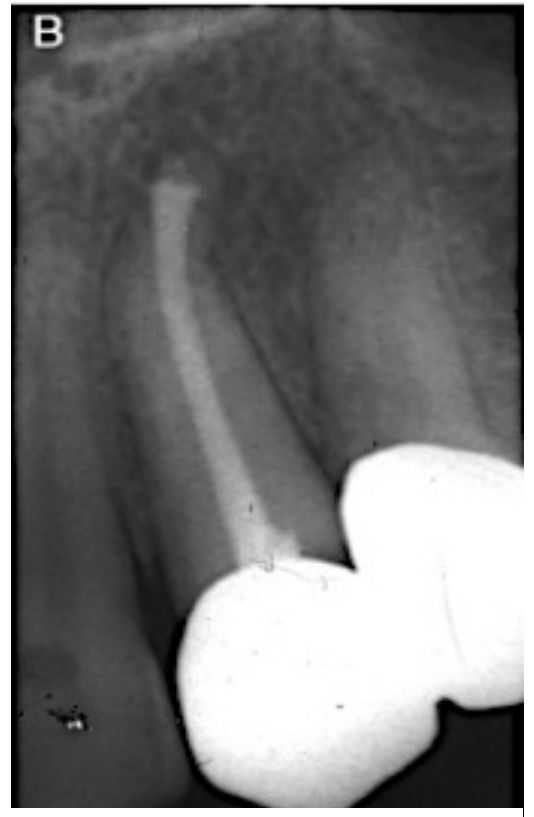

Abb. 1: Radiologische Darstellung einer präparationsbedingten Bildung von zip und elbow; aus Hülsmann et al. (2005). Die Verwendung erfolgt mit freundlicher Genehmigung von John Wiley and Sons.

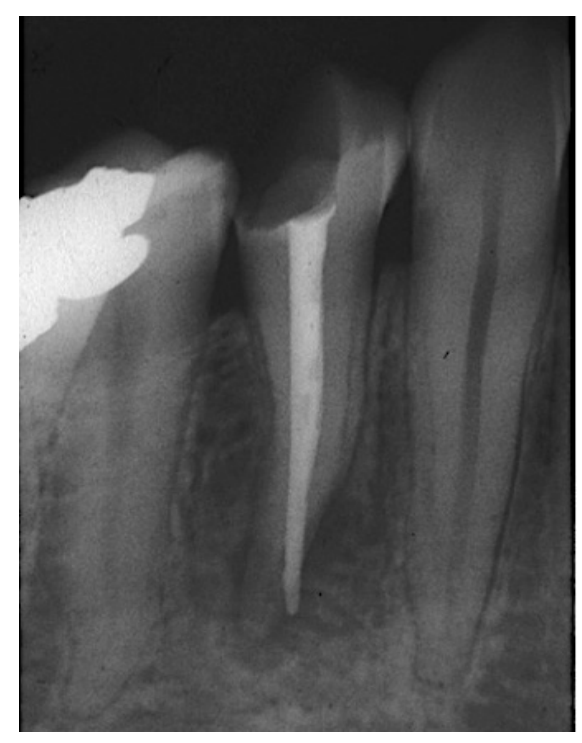

Abb. 3: Radiologische Darstellung einer präparationsbedingten apikalen Perforation; aus Hülsmann et al. (2005). Die Verwendung erfolgt mit freundlicher Genehmigung von John Wiley and Sons.

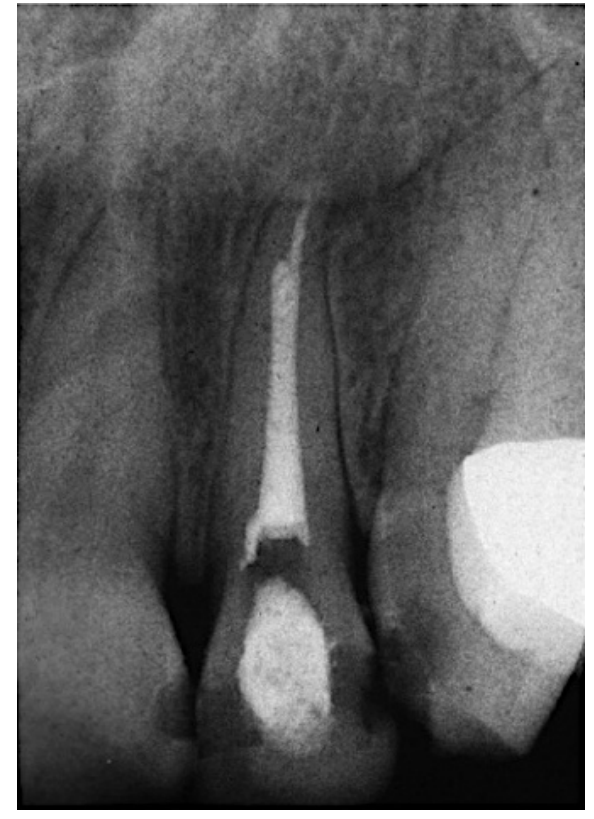

Abb. 2: Radiologische Darstellung einer präparationsbedingten Stufenbildung im apikalen Wurzelkanaldrittel; aus Hülsmann et al. (2005). Die Verwendung erfolgt mit freundlicher Genehmigung von John Wiley and Sons.

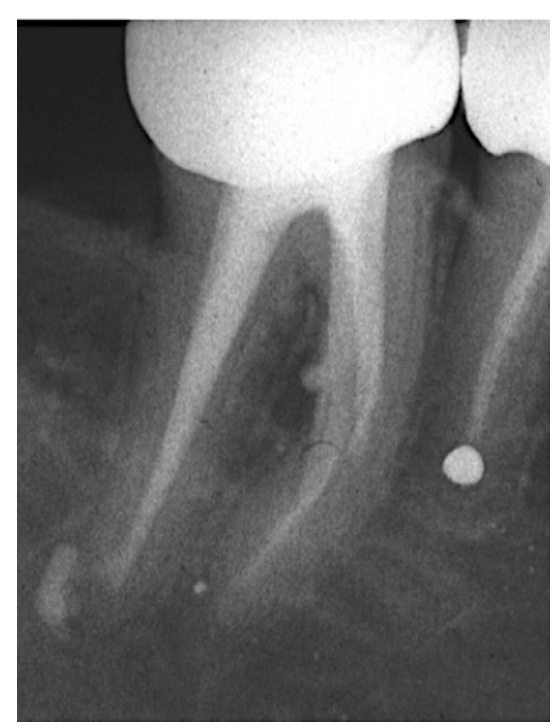

Abb. 4: Radiologische Darstellung einer präparationsbedingten lateralen Perforation; aus Hülsmann et al. (2005). Die Verwendung erfolgt mit freundlicher Genehmigung von John Wiley and Sons. 


\subsection{Maschinelle Wurzelkanalpräparation mit Nickel-Titan- Instrumenten}

Die maschinelle Wurzelkanalpräparation mit NiTi-Legierungen wurde in der Endodontie erstmalig von Walia et al. (1988) beschrieben. Die Legierung selbst wurde bereits Anfang der 1960er Jahre durch das Naval Ordnance Laboratory (White Oak, MD, USA) entwickelt und als Nitinol bezeichnet (Zupanc und Schäfer 2017).

Besondere Eigenschaften dieser Legierung sind die Pseudoelastizität, der shape-memory-Effekt und ein verringertes Elastizitätsmodul (E-Modul) gegenüber herkömmlichen Stahlinstrumenten (Viana et al. 2010; Zupanc und Schäfer 2017). Als shape-memory-Effekt wird das Rückstellvermögen der Feile nach erfolgter Verformung ohne erkennbare plastische Deformation bezeichnet (Zinelis et al. 2010). Pseudoelastizität und ein geringes E- Modul ermöglichen eine zweibis dreifach erhöhte Flexibilität gegenüber Handinstrumenten aus Edelstahl (Hülsmann et al. 2005; Viana et al. 2010). Die Legierung und deren Eigenschaften sind dabei temperaturabhängig (Zhou et al. 2013). Grundlage für die genannten Charakteristika sind die speziellen metallurgischen Eigenschaften. Das Verhältnis von Nickel zu Titan beträgt etwa 56 m\% zu 44 m\% (Thompson 2000). NiTi liegt in zwei verschiedenen Kristallgitterstrukturen vor. Die Hochtemperaturphase (Austenit) ist kubisch angeordnet, die Niedrigtemperaturphase (Martensit) ist monoklin angeordnet (Zupanc und Schäfer 2017). Durch elastische Deformation geht die Austenit- in die Martensitphase über. Eine weitere Deformation würde zu einer Verformung des Materials und anschließend zur Faktur führen. Eine spontane Reversion des spannungsinduzierten Martensits ist bei abbauender Spannung möglich und wird Hysterese genannt (Thompson 2000).

Durch thermomechanische Modifikation und Substitution anderer Elemente wird eine zusätzliche Phase zwischen den beiden Kristallgittern geschaffen, die als rhomboedrische Übergangsphase (R-Phase) bezeichnet wird (Miyazaki und Otsuka 1986; Zupanc und Schäfer 2017). In der R-Phase ist ein Verdrillen der Feile möglich. Auf eine Ausfräsung der gewünschten Feilengeometrie bei der Feilenherstellung und dabei möglich entstehende Mikrofrakturen wird deshalb verzichtet (Zupanc und Schäfer 2017). 


\subsubsection{Rotierend arbeitende Nickel-Titan-Instrumente}

Die ersten rotierend arbeitenden maschinellen NiTi-Feilen wurden Anfang 1990 entwickelt (Haapasalo und Shen 2013). Die Instrumente verfügen über verschiedene Feilengeometrien mit unterschiedlichen Konizitäten. ProFile (Dentsply Sirona, Ballaigues, Schweiz) verwendete abweichend von der ISO-Norm einer 2 \%igen Konizität als eines der ersten Systeme eine Konizität von zunächst $4 \%$ und später von 6 \% (Haapasalo und Shen 2013). Eine effektivere Säuberung des Wurzelkanals konnte durch die Veränderung der Konizität erreicht werden (Peters et al. 2003; Smith et al. 1993). Die Querschnittgeometrie der meisten NiTi-Feilen basieren auf der Geometrie von Reamern mit Schneidwinkeln zwischen $10-30^{\circ}$ und etwa 0,5-1 Verwindungen pro Millimeter (Hülsmann 2008).

\subsubsection{Reziprok arbeitende Nickel-Titan-Instrumente}

Die reziproke Arbeitsweise bei der maschinellen Wurzelkanalpräparation geht auf das 1964 vorgestellte Giromatic-Winkelstück (MicroMéga, Besançon, Frankreich) zurück, welches in einer reziproken Viertelkreisbewegung von $90^{\circ}$ arbeitet (Bürklein et al. 2017; Hülsmann 2008). Da ausschließlich Edelstahlinstrumente verwendet wurden, fielen die Präparationsergebnisse mit ausgeprägten apikalen Veränderungen und Begradigungen des Wurzelkanals eher negativ aus (Peters und Paqué 2010).

Die maschinelle reziproke Anwendung von NiTi-Feilen wurde durch die Versuche von Yared (2008) inauguriert und basiert auf der manuellen balanced-force technique nach Roane et al. (1985). Die Feilen werden dabei mit einer nicht schneidenden Spitze (Batt-Spitze) manuell im Wurzelkanal um $90^{\circ}$ in gegenläufiger Uhrzeigerrichtung aktiv schneidend rotiert, um bei der Gegenbewegung in Uhrzeigerrichtung das abgelöste Dentin von der Kanalwand zu entfernen und abzutransportieren (van der Vyver et al. 2019). Die Präparation des Wurzelkanals mit einer reziproken Bewegung erlaubt die Verwendung einer geringeren Anzahl an Feilen, sodass eine schnellere Präparation gegenüber rotierend arbeitenden NiTi-Systemen mit mehreren Feilensequenzen erfolgen kann (Pedullà et al. 2013).

In dieser Studie werden Feilensysteme verglichen, welche mit lediglich einer Feile den Wurzelkanal in reziproker Arbeitsweise präparieren sollen. Es wurde bereits beschrieben, dass für eine gute Wurzelkanalpräparation mit einem geringen Anteil an unpräparierter Wurzelkanaloberfläche und wenig verbleibendem Debris im Wurzelkanal mehrere Feilen mit unterschiedlichen Konizitäten benötigt 
werden. Es soll überprüft werden, wie gut Einfeilensysteme einen Wurzelkanal präparieren können (Amoroso-Silva et al. 2017; Espir et al. 2017; Robinson et al. 2013). Im Folgenden werden die in dieser Studie verwendeten Feilensysteme vorgestellt.

\subsubsection{Feilensystem RECIPROC}

Das Feilensystem RECIPROC basiert auf dem früher von der Firma VDW (München) entwickelten System Mtwo und wird mit drei unterschiedlichen Feilengrößen verwendet. Alle Feilen bestehen aus der $M$-Wire-NiTi-Legierung. Diese pseudoelastische Legierung wurde 2007 von Dentsply Sirona (Ballaigues, Schweiz) und Sportswire LLC (Langley, OK, USA) entwickelt, um bei erhöhter Flexibilität trotzdem eine höhere Resistenz gegenüber torsionsbedingten Frakturen zu gewährleisten (Zupanc und Schäfer 2017). Eine zusätzliche thermomechanische Behandlung der NiTi-Legierung ermöglicht die verbesserten Eigenschaften, sodass einerseits bei $37^{\circ} \mathrm{C}$ fast gleiche Anteile von Austenit und RPhase vorliegen und zugleich ein geringer Anteil an deformiertem und verzwillingtem Martensit vorliegt (Alapati et al. 2009). Dadurch zeigt $M$-Wire eine erhöhte Flexibilität (Pereira et al. 2012).

Die Feilen haben einen s-förmigen Querschnitt mit 2 Schneidekanten und einer Batt-Spitze (Bürklein et al. 2017). Die erhöhte Resistenz gegenüber Torsionsbrüchen mit erhöhtem Widerstand gegenüber Deformationen resultiert aus einer initial erschwerten Rissbildung durch die vorliegenden Martensit-Varianten (Gao et al. 2010).

Das Set besteht aus den drei Feilen R25 (25/.08), R40 (40/.06) und R50 (50/.05), von denen jeweils nur eine die gesamte Präparation eines Wurzelkanals durchführen soll. Die Präparationsfeilen sind nach der jeweiligen ISO-Größe benannt, weisen aber von der ISO-Norm abweichende Konizitäten auf. Die Konizität ist regressiv gestaltet (Bürklein et al. 2017). Diese beginnt bei RECIPROC-Feilen drei Millimeter von der Instrumentenspitze entfernt als rückläufige Konizität von 4,3\% (Saleh et al. 2015). Die Konizität der ersten drei Millimeter der Feilen liegen bei $8 \%$ (R25), 6 \% (R40) und $5 \%$ (R50) (Bürklein und Schäfer 2013). In dieser Studie wurde ausschließlich die Feile R25 mit einer Länge von $25 \mathrm{~mm}$ verwendet (Abb. 5). Alle Feilen sind zum einmaligen Gebrauch konzipiert. 
Laut Hersteller wird die Auswahl der richtigen Feile anhand der Darstellung am Röntgenbild und der Gängigkeit des Wurzelkanals mit Handinstrumenten ausgewählt. Ist der Wurzelkanal nur teilweise oder gar nicht zu sehen, wird das R25Instrument benutzt. Kann ein Handinstrument der Größe ISO 20 passiv nicht auf Arbeitslänge eingebracht werden, wird ebenfalls die Feile R25 benutzt. Bei radiologisch sichtbarem Wurzelkanal und einem für ein Instrument der Größe 20, nicht aber Größe 30 gängigen Wurzelkanal, wird die R40-Feile verwendet. Bei weiten Kanälen, bei denen ein Handinstrument der Größe ISO 30 passiv auf Arbeitslänge inseriert werden kann, soll die Feile R50 benutzt werden (VDW 2018). Obgleich der Hersteller die Präparation eines Gleitpfades nicht explizit fordert, sollte ein Gleitpfad mit Reamern der Größe ISO 10 und 15 geschaffen werden.

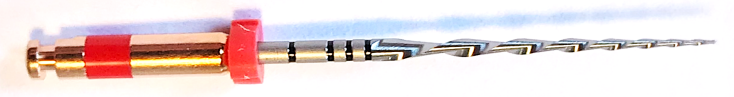

Abb. 5: Feile R25 (25/.08) des Systems RECIPROC

\subsubsection{Feilensystem WaveOne Gold}

Für die Feilen von WaveOne Gold (WOG) wird eine Gold-Wire-Legierung verwendet. Diese ermöglicht durch einen kontrollierten Rückstelleffekt das Vorbiegen der Feile, was durch eine spezielle thermomechanische Behandlung nach dem Schleifen des Instruments erreicht wird (Pereira et al. 2015; Zupanc und Schäfer 2017). Die dabei entstehende goldene Farbe entspricht einer bei der Wärmebehandlung zurückbleibenden Oxidschicht (Hu et al. 2014). Die Legierung besitzt wie die M-Wire-Legierung bei der Transformation zwischen den Martensitund Austenit-Kristallgittern eine R-Phase (Hieawy et al. 2015). Gold-Wire ist weicher und duktiler als $M$-Wire, signifikant flexibler und weniger anfällig gegenüber Biegeermüdungen (De-Deus et al. 2017; Elnaghy und Elsaka 2016; Elsaka et al. 2017; Gao et al. 2012; Gündogar und Özyürek 2017; Özyürek 2016).

Der Querschnitt der Feile entspricht dem eines Parallelogramms und besitzt dabei je nach Zentrierung im Wurzelkanal eine bis zwei Schneidekanten. Die Spitze der Feile ist im Vergleich zu RECIPROC semiaktiv gestaltet. Im Feilensystem WOG können die Feilen mit den entsprechenden Größen und Konizitäten 
Small (20/.07), Primary (25/.07), Medium (35/.06) und Large (45/.05). verwendet werden.

Für die Studie wurde lediglich die Primary-Feile mit der Größe 25 und der regressiven Konizität von 7\% verwendet (Abb. 6).

Obwohl WOG als Einfeilensystem beworben wird, beschreibt der Hersteller ein differenziertes Vorgehen. Ein Gleitpfad soll mit Handfeilen der Größen ISO 10 und ISO 15 geschaffen werden. Die Bedienungsanleitung des Herstellers sieht vor, dass die Primary-Feile das Regelinstrument für die Mehrzahl der Wurzelkanäle darstellt. Ist hiermit ein weiteres Vordringen im sehr engen Wurzelkanal nicht möglich, soll die Small-Feile verwendet werden. Sollte die Primary-Feile in weiten Wurzelkanälen die Arbeitslänge erreichen, aber locker im Wurzelkanal zentriert und die apikalen Bereiche der Feile nicht mit Dentindebris bedeckt sein, soll mit der medium- und large-Feile bis zu größeren apikalen Durchmessern präpariert werden. Die Präparation ist beendet, wenn Dentindebris am apikalen Bereich der Feile zu erkennen ist (Dentsply 2018).

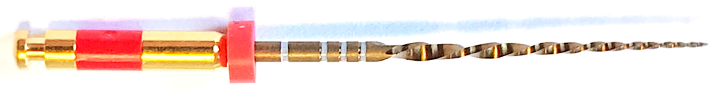

Abb. 6: Feile Primary (25/.07) des Systems WaveOne Gold

\subsubsection{Feilensystem S1 Plus}

Die neuartige S1-Plus-Feile besteht ebenfalls aus einer NiTi-Legierung, die nachträglich wärmebehandelt wurde (Bürklein et al. 2017). Der Querschnitt der Feile ist wie bei RECIPROC s-förmig gestaltet und verfügt über zwei Schneidekanten. Im Gegensatz zur linksschneidenden Feilengeometrie von RECIPROC und WOG ist S1 Plus als rechtsschneidende Feile konzipiert. Durch einen progressiven Steigungswinkel der Schneiden soll der Abtransport von Debris verbessert werden (Sendoline 2018a). Das Feilenset besteht im Vergleich zu den anderen Systemen nur aus den zwei Feilen Standard (25/.06) und Small (15/.06).

Die Standard-Feile ist in Abb. 7 dargestellt und besitzt eine linear gestaltete Konizität von 6\%. S1 Plus wird als Ein-Feilen-System verwendet. Die Small-Feile soll lediglich fakultativ in sehr engen Wurzelkanälen verwendet werden, wenn die 
Präparation mit der Standard-Feile nicht möglich ist. Vor der Präparation wird ein Gleitpfad mit Handinstrumenten bis zur ISO-Größe 15 geschaffen und anschließend mit der Standard-Feile der Wurzelkanal präpariert.

Aufgrund fehlender wissenschaftlicher Studien zu diesem Feilensystem kann noch kein Vergleich zu anderen Feilen vorgenommen oder eine generelle Bewertung abgegeben werden.

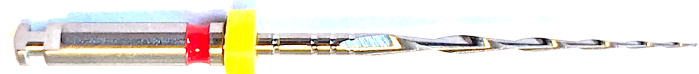

Abb. 7: Feile Standard (25/.06) des Systems S1 Plus

\subsection{Mikro-Computertomographie (Mikro-CT)}

Die Funktionsweise der Computertomographie basiert auf dem Erstellen mehrerer hundert digitaler zweidimensionaler Röntgenbilder des zu untersuchenden Objektes. Die Röntgenquelle kann dabei um das Objekt rotieren (O'Sullivan et al. 2018). Alle 2D-Bilder werden anschließend rechnerisch durch einen Algorithmus zu einem dreidimensionalen Datensatz rekonstruiert (Feldkamp et al. 1984).

Die Weiterentwicklung der Computertomographie als Mikro-Computertomographie (Mikro-CT) mit deutlicher verbesserter Auflösung im Mikrometerbereich wurde Anfang 1980 durch Elliott und Dover (1982) vorgestellt. Konventionelle Computertomographien arbeiten im Vergleich mit Ortsauflösungen von mehreren Millimetern (O'Sullivan et al. 2018). Der Unterschied zur Mikro-CT besteht in der Lagerung des zu untersuchenden Objektes, welches auf dem Objekttisch um 360 Grad rotiert werden kann, wobei die Röntgenquelle starr gelagert ist (Abb. 8). Durch die Verwendung von variablen geometrischen Vergrößerungen können deutlich bessere Auflösungen im Mikrometerbereich erreicht werden (Withers 2007).

Die Anwendung der Mikro-CT in der Endodontie wurde bereits 1990 als nichtdestruktive Methode für In-vitro-Forschungszwecke mit einer Auflösung von etwa $30 \mu \mathrm{m}$ beschrieben (Bergmans et al. 2001; Gluskin et al. 2001; Hübscher et al. 2003; Peters et al. 2000; Tachibana und Matsumoto 1990). Dabei kann die Berechnung von Oberflächen und Volumina in einem komplexen dreidimensionalen Wurzelkanalsystem präzise durchgeführt werden, was von Peters et al. (2000) 
durch die Entwicklung eines neuen Algorithmus ermöglicht wurde. Mit konventionellem Röntgen sind detaillierte Oberflächen- und Volumenberechnungen nicht möglich (Versiani et al. 2013). Mithilfe des Mikro-CT können verschiedene Wurzelkanalpräparationstechniken evaluiert werden (Gambill et al. 1996; Hübscher et al. 2003; Kim et al. 2013; Moore et al. 2009; Paqué et al. 2009). Dabei können durch prä- und postoperativ erstellte Scans verschiedene dreidimensionale Abweichungen vom originären Wurzelkanalverlauf festgestellt und bewertet werden.

A Image acquisition

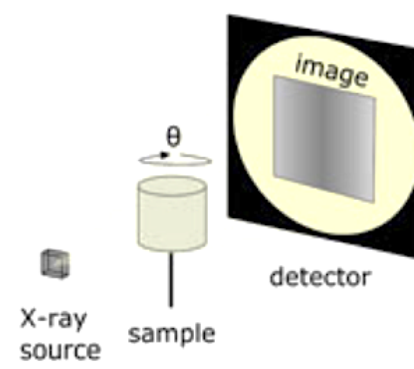

D

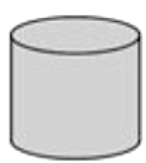

i) $3 \mathrm{D}$ volume rendering

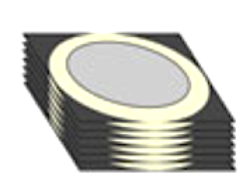

ii) cross-sectional "slices"
B 2D projections of sample

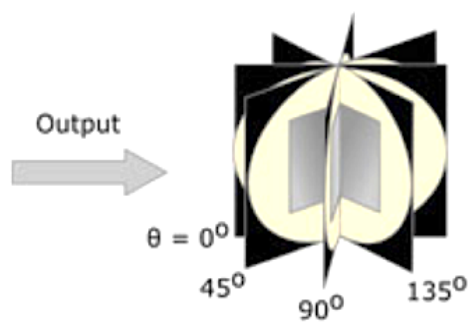

C Reconstruction

1) Filtered backprojection or

2) Iterative reconstruction

Abb. 8: Schematische Darstellung eines Mikro-CT; A: Bilderstellung mit Röntgenquelle, Objekt und Bilddetektor, B: 2D-Projektion des Objektes aus den unterschiedlichen Rotationswinkeln; C: Digitale Rekonstruktion des Datensatzes als iterative Bildrekonstruktion oder gefilterte Rückprojektion; D: Darstellung des rekonstruierten Datensatzes als 3D-Bild oder in Querschnittsbildern; aus O'Sullivan et al. (2018). CC BY 4.0. 


\subsection{Ziel der Studie}

Das Ziel der Ex-vivo-Studie ist der Vergleich der drei NiTi-Feilensysteme RECIPROC, WaveOne Gold und S1 Plus.

Unter standardisierten Bedingungen soll das Präparationsverhalten in Wurzelkanälen mit einfacher und doppelter Krümmung verglichen werden.

Mithilfe der Mikro-CT sollen Volumenveränderungen und Oberflächenveränderungen sowie der Anteil der nicht präparierten Oberfläche der Wurzelkanaloberflächen durch die Wurzelkanalpräparation berechnet werden. Die präparationsbedingten Wurzelkanalbegradigungen mit Berechnung der Canal Transportation und Centering Ability sollen anhand von Querschnittsbildern aufgezeigt werden. Die Veränderung der Form des Wurzelkanalquerschnitts mit Berechnung des Structure Model Index soll dargestellt werden. Abschließend soll die Arbeitssicherheit berücksichtigt werden.

Die Nullhypothese lautete, dass alle Feilensysteme sich bei den untersuchten Parametern gleich verhalten.

Die Ergebnisse der Studie sollen mit den Erkenntnissen aus ähnlichen Studien verglichen werden. 


\section{Material und Methoden}

Die Studie wurde an humanen extrahierten Unterkiefermolaren durchgeführt, die aus Gründen extrahiert wurden, die in keinem zeitlichen oder kausalen Zusammenhang mit der Studie standen. Alle Patienten wurden über den Verwendungszweck der Zähne aufgeklärt und bestätigten schriftlich, ihr biologisches Material für diese Studie bereitzustellen. Die Studie wurde von der Ethik-Kommission der UMG unter der Nr. DOK_127_2016 genehmigt.

Die Zähne wurden nach der Extraktion in einem Sammelgefäß für 3 Stunden in einer 3 \%igen Natriumhypochloritlösung gereinigt und anschließend bei einer konstanten Temperatur von $5^{\circ} \mathrm{C}$ in demineralisiertem Wasser gelagert.

\subsection{Auswahl der Zähne}

Für die Studie wurden 75 Zähne verwendet. Diese mussten folgende Anforderungen erfüllen:

Alle verwendeten Unterkiefermolaren mit intakten mesialen Wurzeln mussten separate mesiale Wurzelkanäle mit abgeschlossenem Wurzelwachstum aufweisen. Dabei sollte die Wurzeloberfläche stets intakt sein und keine bereits begonnene oder abgeschlossene endodontische Behandlung nachweisbar sein. Längsfrakturen mussten ausgeschlossen werden. Die Kronensubstanz musste für ein problemloses Anlegen von Kofferdam ausreichend sein. Die Mindestlänge des Zahns musste $19 \mathrm{~mm}$ betragen. Der Krümmungswinkel aller Versuchszähne musste zwischen $15-40^{\circ}$ liegen und der Krümmungsradius bis zu $18 \mathrm{~mm}$ betragen. Für die spätere Wurzelkanalpräparation mussten alle zu präparierenden Wurzelkanäle gängig sein.

Alle Zähne wurden mithilfe eines diamantierten konischen Schleifers (Hager \& Meisinger, Neuss) unter Wasserkühlung trepaniert und das Pulpakavum dargestellt. Gegebenenfalls vorhandene Dentikel und Dentinüberhänge wurden entfernt. Die Kanaleingänge wurden dargestellt und kontrolliert, ob die mesiolingualen und mesiobukkalen Kanaleingänge getrennt voneinander vorlagen. War dies nicht der Fall, z. B. bei durchgängigen Isthmen, wurden diese Zähne verworfen. Die Gängigkeit der beiden mesialen Wurzelkanäle wurde mit einem Reamer der Größe 10 (Dentsply Sirona, Ballaigues, Schweiz) überprüft. Alle Zähne wurden auf die festgelegte Länge von $19 \mathrm{~mm}$ gekürzt, woraus eine standardisierte Arbeitslänge von $18 \mathrm{~mm}$ resultierte. Bei zu kurzen Versuchszähnen wurde der Zahn mit Komposit (Kulzer, Hanau) auf die gewünschte Arbeitslänge verlängert. Bei 
größeren Hartsubstanzdefekten wurden Schmelzwände ebenfalls mit Komposit wiederaufgebaut, um das Anlegen einer Kofferdamklammer zu ermöglichen.

Die Berechnung von Krümmungswinkel und -radius erfolgte analog dem Vorgehen in früheren Studien mit ähnlicher Fragestellung (De-Deus et al. 2015; Paqué et al. 2005; Pasqualini et al. 2015; Rödig et al. 2012).

Für die Bestimmung des Krümmungswinkels wurden von jedem Versuchszahn vier zweidimensionale Röntgenaufnahmen angefertigt. Es wurden jeweils der mesiobukkale und der mesiolinguale Wurzelkanal in mesiodistaler und bukkolingualer Richtung geröntgt. Die Aufnahme erfolgte in Rechtwinkeltechnik bei einer Belichtungsdauer von 0,05 Sekunden und einer Röhrenspannung von 60 kV (Heliodent Plus (Dentsply Sirona, Bensheim)). Für die Auswertung der Aufnahmen wurde neben den Versuchszähnen ein $10 \mathrm{~mm}$ langer röntgenopaker Metallstift mit abgebildet, der als Abbildungsmaßstab diente (Abb. 9 und Abb. 10). Somit konnte die softwareunterstützte Berechnung von Krümmungswinkel und -radius durch das Computerprogramm ImageJ (National Institutes of Health, Washington DC, USA) durchgeführt werden, das für die Berechnung des Krümmungswinkels auf den Methoden nach Schneider (1971) und für die Berechnung des Krümmungsradius auf der Methode nach Schäfer et al. (2002) beruht.
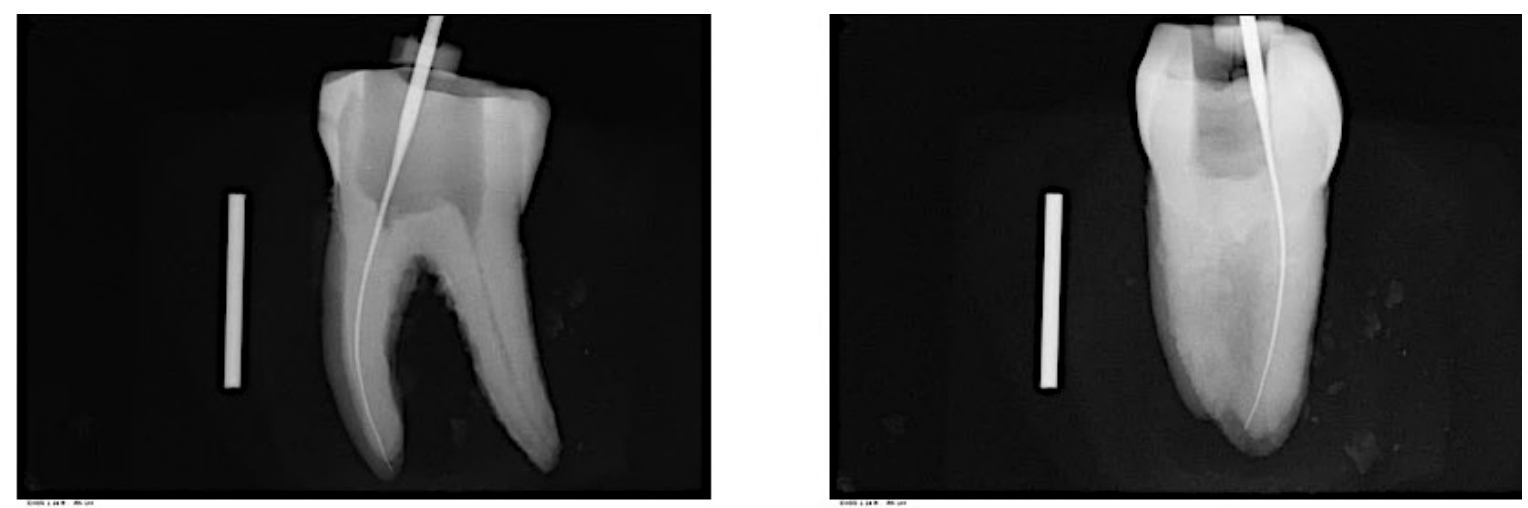

Abb. 9: Röntgenmessaufnahme eines mesiobukkalen Wurzelkanals; eingelegter Reamer ISO 10 in bukkolingualer Darstellung (linkes Bild) und mesiodistaler Darstellung (rechtes Bild) sowie Darstellung des röntgenopaken Abbildungsmaßstabes 

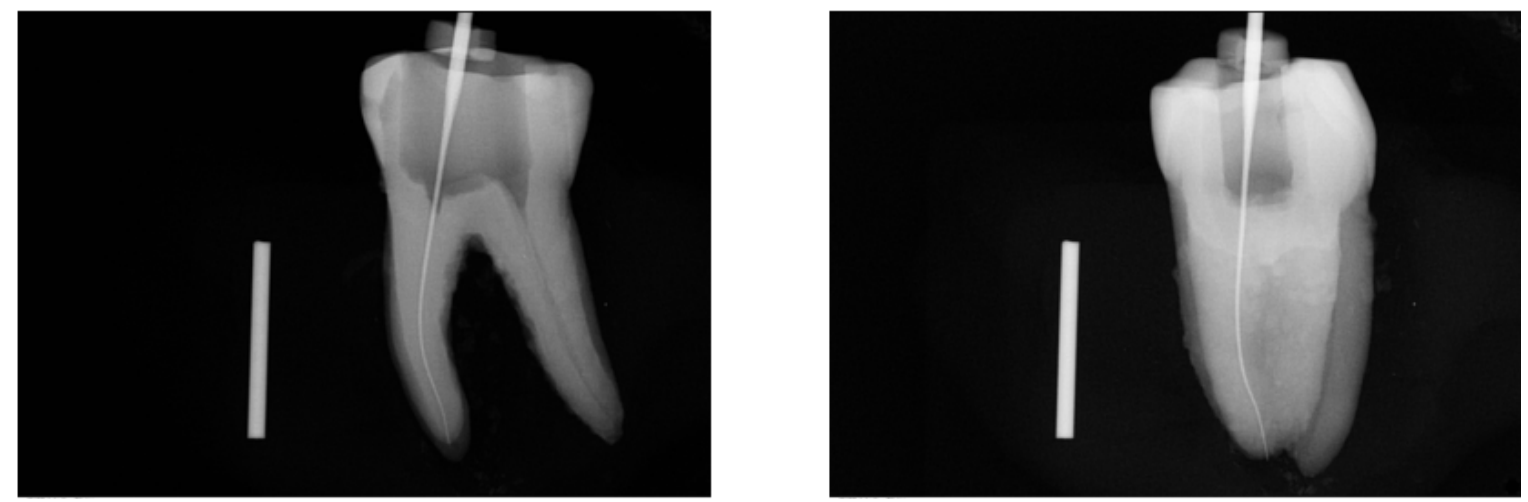

Abb. 10: Röntgenmessaufnahme eines mesiolingualen Wurzelkanals; eingelegter Reamer ISO 10 in bukkolingualer Darstellung (linkes Bild) und mesiodistaler Darstellung (rechtes Bild) sowie Darstellung des röntgenopaken Abbildungsmaßstabes

Zur Berechnung des Krümmungswinkels wurde eine Längsachse am Röntgenbild durch den geraden koronalen Anteil des Wurzelkanals gelegt. Der Punkt, an dem der Wurzelkanalverlauf diese Achse verlässt, wurde als Punkt A markiert. Punkt B wurde durch den röntgenologischen Apex gekennzeichnet. Der Winkel a entsteht zwischen der Verbindungslinie zwischen Punkt A und $B$, also Linie AB, und der Längsachse des Wurzelkanals (Abb. 11).

Beim Krümmungsradius sollte die Linie $|A B|$ auf einer gedachten Kreissehne $S$ liegen. Dabei liegt die Längsachse tangential an Punkt A an, der an der gedachten Kreisbahn liegt. Mit folgender Formel ließ sich der Krümmungsradius aus den vorliegenden Parametern errechnen:

$$
r=\frac{|A B|}{2 \cdot \sin \alpha}
$$

Die Strecke $|A B|$ ist der Abstand zwischen Punkt A und B in Millimetern (Abb. 12). Sofern eine zweite Krümmung im weiteren Verlauf des Wurzelkanals auftrat, wurde adaptiert an die Technik nach Cunningham und Senia (1992) der zweite Krümmungswinkel ebenfalls berechnet (Abb. 13). Die aus den Verbindungslinien $|a b|$ und $|b c|$ sowie $|b c|$ und $|c d|$ resultierenden Winkel entsprechen der Berechnung der Krümmungswinkel nach Schneider (1971). 


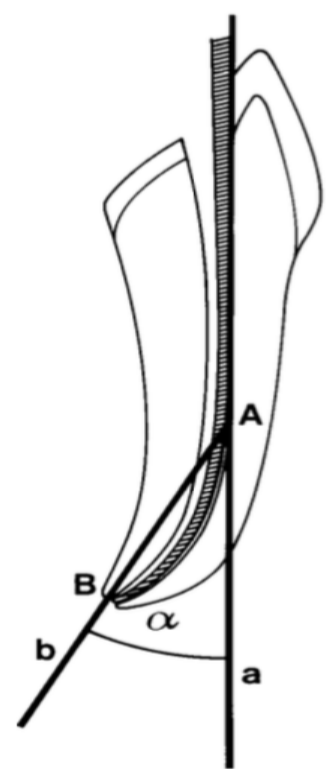

Abb. 11: Messung des Krümmungswinkels nach Schneider (1971); $\alpha=$ Krümmungswinkel; $\mathrm{A}=$ Beginn der Wurzelkanalkrümmung; $\mathrm{B}=$ apikales Foramen; $\quad \mathrm{a}=$ Tangente des Wurzelkanalverlaufs vor der ersten Wurzelkanalkrümmung; $b=$ Verbindungslinie zwischen den Punkten A und B; aus Schäfer et al. (2002). Die Verwendung erfolgt mit freundlicher Genehmigung von Elsevier.

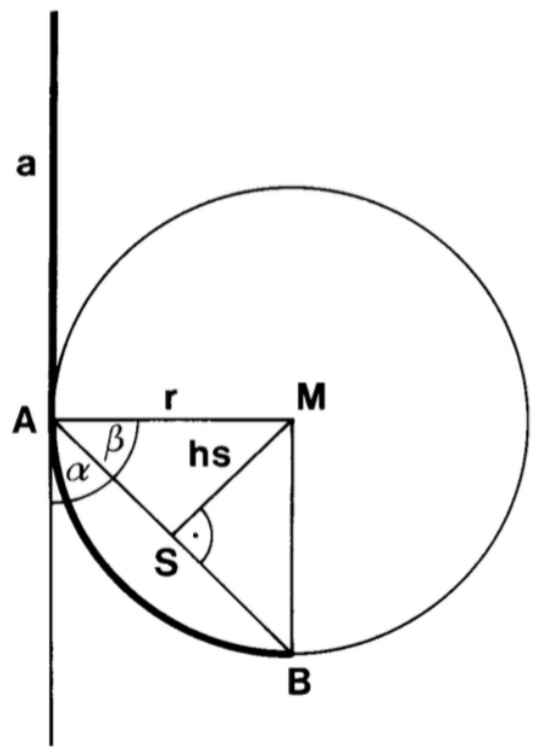

Abb. 12: Berechnung des Krümmungsradius nach Schäfer et al. (2002); A = Beginn der Wurzelkanalkrümmung; $B=$ apikales Foramen; $\mathrm{B}=$ apikales Foramen; $\mathrm{S}=$ gedachte Kreissehne; $M=$ Mittelpunkt der gedachten Kreisbahn; $r=$ Kreisradius; $\alpha=$ Krümmungswinkel; $\beta=$ Winkel $+\alpha=90^{\circ}$; aus Schäfer et al. (2002). Die Verwendung erfolgt mit freundlicher Genehmigung von Elsevier.

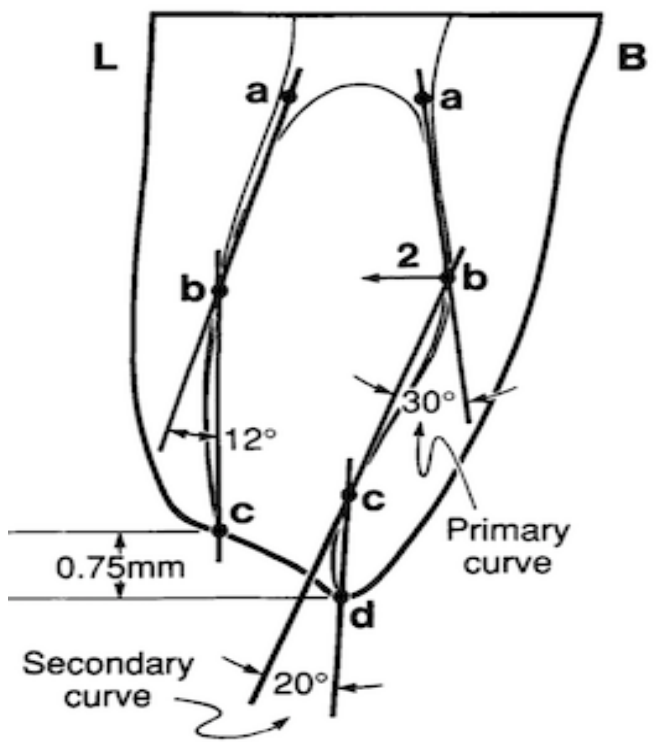

Abb. 13: Berechnung einer zweiten Wurzelkanalkrümmung nach Cunningham und Senia (1992); L: linguale Seite; B: bukkale Seite; a: Beginn Wurzelkanal; b: Beginn der ersten Wurzelkanalkrümmung; c: apikales Foramen des lingualen Wurzelkanals, Beginn der zweiten Wurzelkanalkrümmung des bukkalen Wurzelkanals; d: apikales Foramen des bukkalen Wurzelkanals; 2: Vektor in die Richtung der zu erwartenden Canal Transportation; aus Cunningham und Senia (1992). Die Verwendung erfolgt mit freundlicher Genehmigung von Elsevier. 


\subsection{Einteilung der Versuchsgruppen}

Für die Versuche wurde für jedes der drei Feilensysteme eine Hauptgruppe mit 25 Versuchszähnen gebildet. Die Hauptgruppen wurden zudem in zwei Untergruppen mit einer einfachen Krümmung und einer doppelten Krümmung in der Wurzelkanalanatomie aufgeteilt. Der Anteil von Versuchszähnen mit einfacher Krümmung lag in allen Hauptgruppen bei 14, der Anteil mit doppelter Krümmung bei 11 Zähnen. Ob eine doppelte Krümmung vorlag, wurde initial durch die vier beschriebenen Röntgenkontrollen an jedem Versuchszahn bestimmt (siehe Kapitel 2.1). Lag bei mindestens einem der vier Röntgenbilder eine doppelte Krümmung vor, wurde der Versuchszahn der entsprechenden Untergruppe mit einer doppelten Krümmung zugeordnet. Die Versuchszähne wurden so verteilt, dass ein nahezu identischer Mittelwert von Krümmungswinkel und -radius zwischen den drei Hauptgruppen vorhanden war (Tab. 1, Tab. 2).

Für die Präparation und spätere Analyse wurde jeweils ein Drilling mit einem Versuchszahn mit vergleichbaren Wurzelkrümmungen und -winkeln gebildet (matched triples). Die Zuordnung jedes Zahnes der Dreiergruppe zu der entsprechenden Hauptgruppe erfolgte randomisiert. Zwischen Versuchszähnen mit doppelter Krümmung und einfacher Krümmung wurde dabei strikt unterteilt, sodass nur Drillinge mit entweder einfacher oder doppelter Krümmung vorlagen. Alle Zähne wurden anschließend durch die Gruppeneinteilung jeweils einem der drei Feilensysteme zugeordnet

Tab. 1: Mittelwerte der Krümmungswinkel und Krümmmungsradien der mesiobukkalen Wurzelkanäle in bukko-lingualer und mesio-distaler Ansicht

\begin{tabular}{|c|c|c|c|c|}
\hline \multirow{2}{*}{ Feilengruppe } & \multicolumn{4}{|c|}{ mesiobukkaler Kanal } \\
\cline { 2 - 5 } & \multicolumn{2}{|c|}{ bukko-lingual } & \multicolumn{2}{|c|}{ mesio-distal } \\
\cline { 2 - 5 } & Winkel $\left[{ }^{\circ}\right]$ & Radius [mm] & Winkel $\left.{ }^{\circ}{ }^{\circ}\right]$ & Radius [mm] \\
\hline S1 Plus & 24,1 & 10,3 & 18,1 & 11,4 \\
\hline RECIPROC & 24 & 10,3 & 17,5 & 10,8 \\
\hline WaveOne Gold & 23,9 & 10,1 & 17,8 & 11,4 \\
\hline
\end{tabular}


Tab. 2: Mittelwerte der Krümmungswinkel und Krümmmungsradien der mesiolingualen Wurzelkanäle in bukko-lingualer und mesio-distaler Ansicht

\begin{tabular}{|c|c|c|c|c|}
\hline \multirow{2}{*}{ Feilengruppe } & \multicolumn{4}{|c|}{ mesiolingualer Kanal } \\
\cline { 2 - 5 } & \multicolumn{2}{|c|}{ bukko-lingual } & \multicolumn{2}{c|}{ mesio-distal } \\
\cline { 2 - 5 } & Winkel $\left.{ }^{\circ}{ }^{\circ}\right]$ & Radius [mm] & Winkel [ $\left.{ }^{\circ}\right]$ & Radius [mm] \\
\hline S1 Plus & 22,9 & 10,1 & 18,3 & 11,4 \\
\hline RECIPROC & 22,9 & 10,3 & 18 & 10,9 \\
\hline WaveOne Gold & 23,2 & 10,3 & 18,1 & 11,1 \\
\hline
\end{tabular}

\subsection{Versuchsaufbau}

Vor Beginn der Präparation wurde mit einem Reamer der Größe ISO 10 (Dentsply Sirona, Ballaigues, Schweiz) bei allen Zähnen die Gängigkeit des Wurzelkanals überprüft und mit einem Reamer der Größe ISO 15 (Dentsply Sirona, Ballaigues, Schweiz) ein Gleitpfad für den späteren Gebrauch der NiTi-Feilen geschaffen. Alle Zähne wurden von einem Zahnarzt präpariert.

\subsubsection{Spülprotokoll}

Beim einheitlichen Spülprotokoll für alle Versuchsgruppen wurde während der Präparation nach jedem Arbeitszyklus einer Feile der Wurzelkanal mit 2 ml Natriumhypochlorit in $3 \%$ iger Konzentration gespült. Als letzte Spülung wurde nach abgeschlossener Präparation der Wurzelkanal erneut mit 1 ml Natriumhypochlorit (3\%) befüllt und die Spüllösung zusätzlich aktiviert. Dabei wurde das luftbetriebene Handstück Sonicflex 2003 L (Kavo, Biberach) mit Eddy (VDW, München), einer Kunststoffspitze zur Aktivierung von Spülflüssigkeiten, kombiniert. Diese wurde bei einer maximalen Frequenz von $6000 \mathrm{~Hz}$ und der geringsten Amplitudeneinstellung von $120 \mu \mathrm{m}$ verwendet. Eddy wurde maximal bis auf $2 \mathrm{~mm}$ vor Arbeitslänge, also auf $16 \mathrm{~mm}$ in den Wurzelkanal eingebracht und für $10 \mathrm{~s}$ aktiviert. Diese Abfolge wurde insgesamt dreimal durchgeführt. Anschließend wurde eine Spülung mit $1 \mathrm{ml} 10$ \%iger Zitronensäure verwendet, die gleichermaßen für $10 \mathrm{~s}$ aktiviert wurde; dieser Zyklus wurde insgesamt dreimal wiederholt. Im letzten Schritt wurde der Wurzelkanal mit 3 ml Natriumhypochloritlösung (3\%) gespült. 


\subsubsection{Wurzelkanalpräparation mit S1 Plus}

Das Einfeilensystem S1 Plus arbeitet mit dem firmeneigenen Winkelstück S1-E. Die reziproke Bewegung entsteht durch einen Rotationswinkel von $180^{\circ} \mathrm{im}$ Uhrzeigersinn und einer folgenden gegenläufigen Bewegung von $30^{\circ}$. Ein externer Endodontiemotor wurde nicht benötigt. Das S1-E-Winkelstück wurde mit einer Übersetzung von 1:150 bei einer Umdrehung bis $40.000 \mathrm{Umin}^{-1}$ verwendet. Über ein integriertes Getriebe wird die reziproke Bewegung bei einer mittleren Drehzahl von 270 Umin $^{-1}$ erzeugt (Sendoline 2018b).

Die Feile der Größe 25/.06 wurde in reziproker Bewegung mit leichtem Druck in den Wurzelkanal eingebracht und in einer Auf- und Abbewegung mit einer Amplitude von $3 \mathrm{~mm}$ bewegt, bis ein Widerstand gespürt wurde. Dieser Zyklus wurde dreimal wiederholt, um danach den Wurzelkanal mit Natriumhypochlorit (3 \%) zu spülen und die NiTi-Feile mit einer sterilen Gaze zu reinigen. Dieses Procedere wurde solange wiederholt, bis die Arbeitslänge erreicht war.

\subsubsection{Wurzelkanalpräparation mit WaveOne Gold}

Das Einfeilensystem WOG benötigte für die Wurzelkanalpräparation einen speziell für dieses Feilensystem programmierten externen Endodontiemotor (WaveOne-Endodontie-Motor (Dentsply Sirona, Ballaigues, Schweiz)), der mit einem Drehmoment von $4 \mathrm{Ncm}$ und einer reziproken Rotationsgeschwindigkeit von 350 $U_{\text {min }}^{-1}$ im Modus „WaveOne All“ verwendet wurde. Der Endodontiemotor arbeitet mit abwechselnden Rotationswinkeln von $150^{\circ}$ gegen den Uhrzeigersinn und $30^{\circ}$ mit dem Uhrzeigersinn (Bürklein et al. 2017). Die Feile schneidet dabei aktiv bei der Bewegung im Gegenuhrzeigersinn. Die Rotation im Uhrzeigersinn entlastet hingegen die Torsionsspannung auf die Feile. Das Winkelstück arbeitet dabei mit einer Übersetzung von 6:1. Bei einer Drehmomentüberschreitung wurde automatisch die Bewegung unterbrochen. In dieser Studie wurde ausschließlich die Primary-Feile der Größe 25/.07 verwendet, die einen Durchmesser von 0,25 mm an der Instrumentenspitze, aber eine Konizität von 7 \% aufweist. Der Präparationsablauf entsprach dem Vorgehen wie in Kapitel 2.3.2.

\subsubsection{Wurzelkanalpräparation mit RECIPROC}

Wie bei WOG wurde beim Feilensystem RECIPOC ein externer Endodontiemotor verwendet (WaveOne-Endodontie-Motor (Dentsply Sirona, Ballaigues, Schweiz)), um die reziproke Bewegung des Winkelstücks zu ermöglichen. Dabei 
wurde der Modus „Reciproc All“ gewählt, der pro Sekunde 10 reziproke Arbeitszyklen erreicht. Der Modus arbeitet mit 300 Umin $^{-1}$ (Özyürek 2016). Es wurde mit der Feile R25 (25/.08) mit einem Durchmesser von 0,25 mm an der Instrumentenspitze und einer apikalen Konizität von $8 \%$ gearbeitet, die nach $3 \mathrm{~mm}$ in eine 5 \%ige Steigung übergeht. Somit weist diese Feile im Vergleich mit den beiden Instrumenten die höchste Konizität auf. Der Präparationsablauf entspricht dem Vorgehen wie in Kapitel 2.3.2.

\subsection{Mikro-CT-Messung}

Von allen Versuchszähnen wurden präoperativ Mikro-CT-Aufnahmen mit dem Gerät Bruker SkyScan 1272 (Bruker Corporation, Kontich, Belgien) erstellt (Abb. 15). Das Gerät kann Objekte bis zu einer Größe von 75 mm Durchmesser scannen. Die Strahlungsquelle kann eine Röhrenspannung von 20-100 kV aufbauen. Für die Messungen stehen sechs verschiedene Metallfilter zur Auswahl (Bruker 2018). Eine Auflösung bis zu 209 Megapixel (14450 x 14450 Pixel) kann erreicht werden. Objektdetails von 0,35 $\mu \mathrm{m}$ können mithilfe einer Phasenkontrastverstärkung dargestellt werden (Bruker 2018).

Für die Scans wurden die Proben in einem nicht röntgenopaken Messzylinder aufbewahrt und mit Schaumstoff ummantelt, um ein Verrutschen der Probe während des Scanvorgangs zu vermeiden (Abb. 14). Pro Messung konnten zwei Zahnproben im Messzylinder aufbewahrt werden.

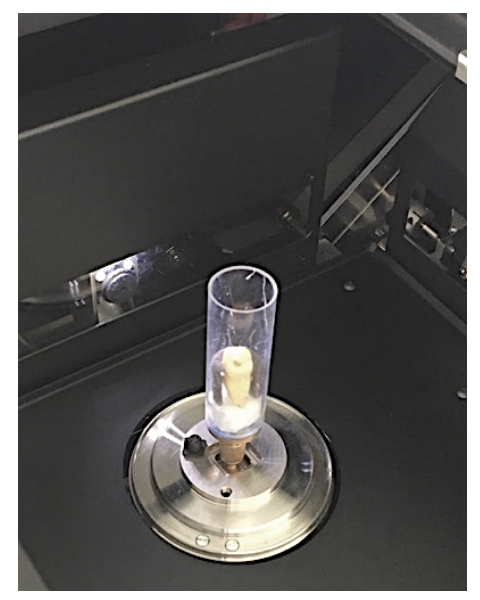

Abb. 14: Versuchszahn im nicht röntgenopaken Messzylinder

Jeder Scan wurde mit vorab festgelegten Einstellungen durchgeführt. Die Zahnproben wurden bei einer durchschnittlichen Spannung von $90 \mathrm{kV}$ und einer Stromstärke von $111 \mu \mathrm{A}$ gescannt. Die Pixelgröße betrug bei allen Messungen 10,5 $\mu \mathrm{m}$. Die Auflösung wurde auf 2452 x 1640 Pixel festgelegt. Die Scanzeit 
jeder Probe betrug $1 \mathrm{~h} 12 \mathrm{~min} 38 \mathrm{~s}$. Ein Filter aus $0,5 \mathrm{~mm}$ dickem Aluminium und $0,038 \mathrm{~mm}$ dickem Kupfer wurde benutzt. Der Versuchskörper wurde insgesamt $188^{\circ}$ um die vertikale Achse gedreht. Dabei wurde immer ein Röntgenbild mit einem Abstand eines Rotationsschrittes von $0,4^{\circ}$ angefertigt.

Insgesamt entstanden somit pro Messung 471 zweidimensionale Bilder, welche anschließend mit der Software SkyScan1272 Version 1.1.7 (Bruker Corporation, Kontich, Belgien) zusammengerechnet wurden. Die Dateigröße pro Messung betrug 3,45 Gigabyte, welche als tagged image file (tif) abgespeichert wurde. Die Datensätze als dreidimensionales Bild wurden mit der Software DataViewer Version 1.5.6.1 (Bruker Corporation, Kontich, Belgien) visualisiert.

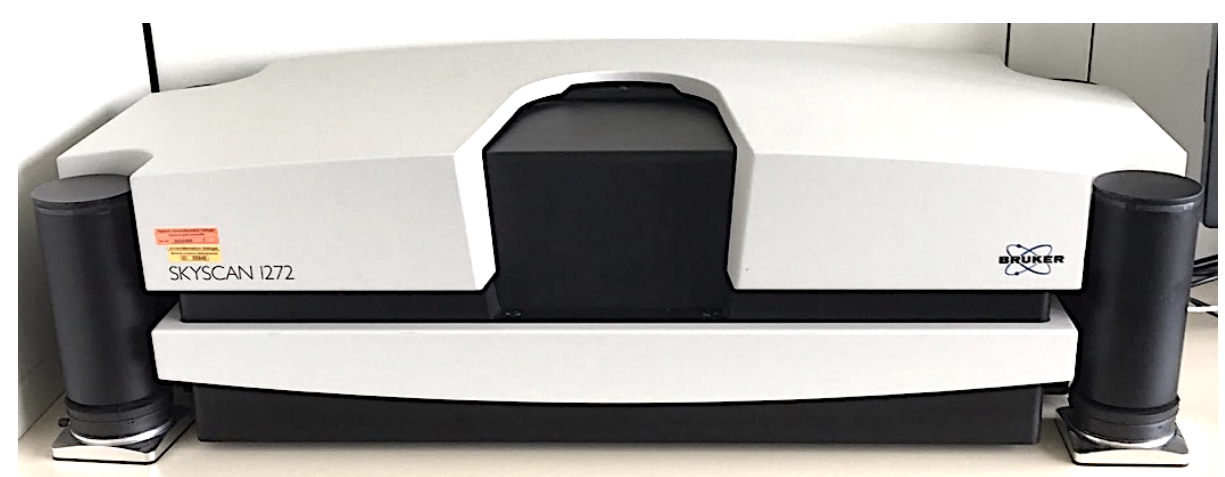

Abb. 15: Mikro-CT Bruker SkyScan 1272

\subsection{Mikro-CT-Analyse}

\subsubsection{Rekonstruktion der Datensätze}

Postoperativ wurden alle Versuchszähne erneut mit den gleichen Parametern wie bei den präoperativen Aufnahmen gescannt und mit der Software NRecon Version 1.7.0.3 (Bruker Corporation, Kontich, Belgien) bearbeitet, um aus den Projektionsbildern aus dem Scan einen Datensatz aus Querschnittsbildern zu rekonstruieren (Bruker 2016). Das Dateiformat wurde als portable network graphics (png) ausgegeben.

Neben der Darstellung als Querschnittsbilder wurden beim Scan potentiell entstandene Artefakte und Abweichungen durch die Feineinstellung verschiedener Parameter entfernt. Für die Feineinstellung wurden die vier Parameter post-alignment, beam hardening correction, ring artefacts reduction und smoothing zur manuellen Einstellung bearbeitet. 
Das post-alignment entspricht der Korrektur einer möglichen Fehlausrichtung des Objekts während des Scans. Die Fehlausrichtung würde zu Doppelbildern, Artefakten oder Unschärfe des Bildes führen (Bruker 2016). Durch leichte Messunterschiede zwischen den Datensätzen wurde für jeden Scan durch visuelle Einsicht eine individuelle Korrektur der Ausrichtung vorgenommen. Alle Scans wurden in der Software in der Feineinstellung für das post-alignment in einem Wertebereich zwischen 13 und 19 korrigiert.

Artefakte durch die radiologische Bildgebung bewirken eine Diskrepanz zwischen dem tatsächlichen Objekt und dem rekonstruierten Bild (Schulze et al. 2010). Die Artefakte entstehen, wenn Photonen mit niedriger Energie aus dem polychromatischen Röntgenstrahl von strahlenundurchlässigen Materialien absorbiert werden und anschließend mit höherer Energie als der zuvor einfallende Strahl den Detektor erreichen (Barrett und Keat 2004).

Der als beam hardening (Strahlungshärtung) benannte Prozess führt zu Artefakten in Form von Streifen und Verzerrungen auf dem Bild (Barrett und Keat 2004; Fox et al. 2018). Strahlungshärtungsartefakte wurden mit der Software NRecon korrigiert. Die Korrekturtiefe wurde für alle Scans auf 60 festgelegt.

Durch fehlerhafte Detektorpixelelemente können Artefakte auf das Bild projiziert werden, welche sich durch die Rotation des Scanobjektes um $360^{\circ}$ - oder $180^{\circ}$ als Ringe oder Halbringe im späteren Bild manifestieren (Yousuf und Asaduzzaman 2010). Die Ringartefakte wurden in der Software reduziert. Es wurden für alle Scans Werte zwischen 11 und 15 ausgewählt.

Im letzten Schritt der Rekonstruktion wurde jedes Pixel in horizontaler und vertikaler Ebene geglättet (smoothing) (Bruker 2016). Bei zu starker Glättung können randständig an jedem Pixel Unschärfen auftreten, weswegen die Glättungsstufe in der Software NRecon in der Feineinstellung angepasst wurde. Für alle Scans wurde die Stufe drei ausgewählt.

\subsubsection{D-Registrierung}

Für die genaue dreidimensionale Berechnung von Oberflächen und Volumina wurden die prä- und postoperativen Scans mit der Software DataViewer übereinander gelagert. Mit dem Befehl 3D-registration wurde der postoperative Scan als Referenz festgelegt und der präoperative Scan auf diesen rotiert. Dabei wurde das Objekt in dem Programm in allen drei Dimensionen angezeigt und mithilfe des Reiters register all aneinander für jede Dimension angepasst. Bei geringen visuell einsehbaren Registrierfehlern konnten die Überlagerungen manuell nachjustiert werden, bis kein Unterschied zwischen beiden Bildern mehr zu sehen war. 
Die registrierten Datensätze wurden in einem separaten Ordner mit der Bezeichnung reference für die präoperativen Bilder und target für die postoperativen Bilder abgespeichert.

\subsubsection{Volumenbestimmung}

Die Volumenbestimmung wurde mit der Software CT-Analyser Version 1.16.4.1 (Bruker Corporation, Kontich, Belgien) durchgeführt. Als region of interest (ROI) wurde im dreidimensionalen Bild des postoperativen Scans der Wurzelkanal vom Apex minus einem Millimeter bis zur Schmelz-Zementgrenze ausgewählt, ohne dass Luftbereiche außerhalb des Zahnes zu sehen waren. Die ausgewählte ROI wurde auf den präoperativen Scan übertragen.

Ein Histogramm für die im ROI dargestellten Grauwertabstufungen wurde erstellt. Dabei entsprach der Grauwert der jeweiligen Dichte des Zahnes und wurde vom Programm als grayscale index bezeichnet. Für die genaue Darstellung der Dichte von Dentin und Luft im Wurzelkanal, wurden Indexwerte von 64 für die präoperativen und 103 für die postoperativen Scans ausgewählt. Der Datensatz wurde durch den Befehl custom processing (benutzerdefinierte Verarbeitung) in ein binäres Bild umgewandelt, wobei die Luft weiß und Dentinanteile schwarz dargestellt wurden. Das Dentin wurde ausgeschnitten, um nur das Volumen der Luft, welche dem Volumen des Wurzelkanals entspricht, darzustellen. Mit dem Reiter despeckle wurden weiße und schwarze Pixelfehler bis zu einer Größe von 100 Pixeln entfernt und das Volumen mit 3D-analysis in Kubikmillimetern berechnet. Die Volumendifferenz wurde durch Subtraktion des präoperativen Wertes vom postoperativen Wert ausgerechnet und als Prozentwert wiedergeben.

Die präparationsbedingt veränderten Wurzelkanäle wurden zusätzlich zur Anschauung mit der Software DataViewer dreidimensional visualisiert. Hierbei wurden die präoperativen Wurzelkanalverläufe farbig markiert und in die Rohdatei des gesacnnten Zahnes eingefügt, um den Wurzelkanal im Versuchszahn sichtbar zu machen. Eine zusätzliche Überlagerung mit dem postoperativen Wurzelkanalverlauf war ebenfalls möglich und wurde zur Anschauung durchgeführt (Abb. 16-Abb. 18). Vorliegende doppelte Krümmungen konnten mit der Software dreidimensional dargestellt werden (Abb. 17, Abb. 18). 

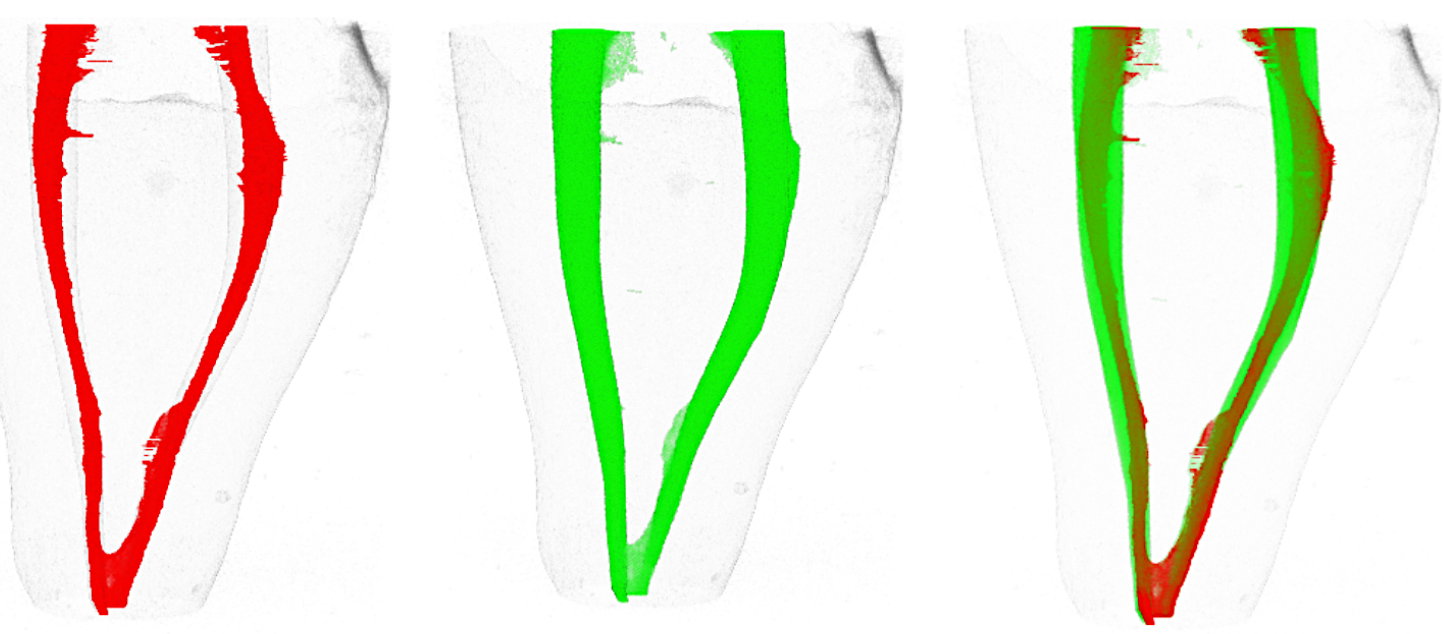

Abb. 16: 3D-Darstellung eines Versuchszahns in mesio-distaler Ansicht; farbig markiertes Wurzelkanalvolumen (rot: präoperatives Wurzelkanalvolumen; grün: postoperatives Wurzelkanalvolumen)

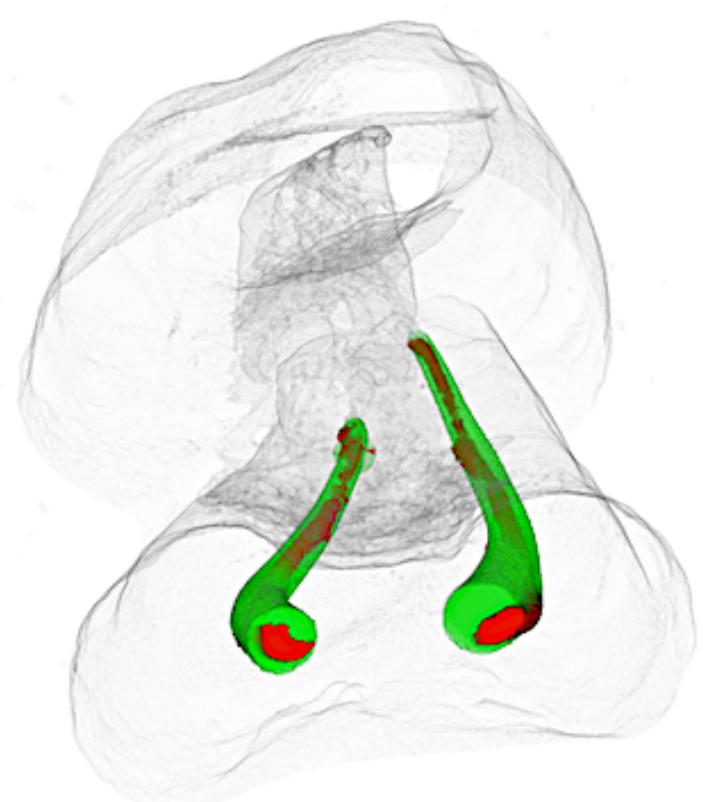

Abb. 17: 3D-Darstellung eines Versuchszahns mit s-shape-Wurzelkanalverlauf in koronal-apikaler Ansicht; farbig markierte mesiale Wurzelkanäle (rot: präoperativer Wurzelkanal; grün: postoperativer Wurzelkanal) 


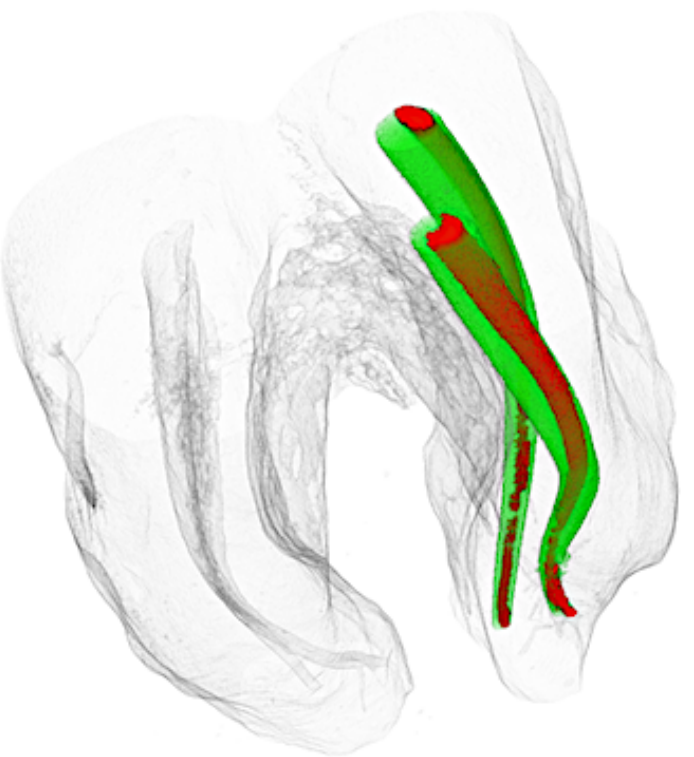

Abb. 18: 3D-Darstellung eines Versuchszahns mit s-shape-Wurzelkanalverlauf in bucco-lingualer Ansicht; farbig markierte mesiale Wurzelkanäle (rot: präoperativer Wurzelkanal; grün: postoperativer Wurzelkanal)

\subsubsection{Oberflächenbestimmung}

Die in Kapitel 2.5.3 ausgerechneten Datensätze der Volumenbestimmung wurden weiterverwendet, um die Oberflächenbereiche des Wurzelkanals mit 3Danalysis mit der Software CT-Analyser zu berechnen. Wie auch bei der Volumenbestimmung, wurden die nicht präparierten interkanalären Isthmen mit in die Rechnung einbezogen. Die Oberflächendifferenz wurde durch Subtraktion des präoperativen Wertes vom postoperativen Wert errechnet und als $\Delta$ Surface als absolute Zahl und Prozentwert angegeben.

Zur Berechnung der unpräparierten Oberfläche wurden die Scans in der Software CT-Analyser überlagert dargestellt, indem das postoperative Bild als Referenzdatei ausgewählt und das präoperative Bild als ROI geöffnet wurde. Über den Reiter morphological operations wurde das Bild so verändert, dass nur noch die Grenzen der Kanalwände in Form eines weißen Ringes mit einer Dicke von drei Pixeln zu sehen waren. Der Unterschied zwischen präparierter und unpräparierter Fläche konnte somit sichtbar gemacht werden. Um die unpräparierte Fläche einzeln anzuzeigen, wurde vom Referenzbild (postoperativ) die ROI über den Reiter bitwise operations mit dem Befehl image = image sub ROI subtrahiert. Die erhaltene Oberfläche wurde über morphological operations in der Dicke auf einen 
Pixel verringert und mit 3D-analysis berechnet. Die berechnete Oberfläche entsprach dem inneren und äußeren Bereich der angezeigten Kanalwandgrenze mit der Breite eines Pixels, was dem Doppelten des zu erzielenden Wertes gleichkommt. Als letzter Schritt wurde der errechnete Wert durch zwei dividiert, um die unpräparierte Fläche anzuzeigen.

\subsubsection{Structure Model Index}

Der Structure Model Index (SMI) gibt eine Bewertung des dreidimensionalen Wurzelkanals an. Dieser beruht auf dem von Hildebrand und Rüegsegger (1997a) vorgestellten mathematischen Modell, welches das Volumen und die Oberfläche, ursprünglich angewendet an trabekulären Knochenstrukturen, bewerten kann. Der SMI wird mit folgender Formel berechnet

$$
S M I=6 \cdot \frac{S^{\prime} \cdot V}{S^{2}}
$$

Dabei entspricht S der Oberfläche vor der Oberflächenveränderung und S' der nach Dilatation entstandenen Oberfläche. $V$ steht für das initial unveränderte Ausgangsvolumen (Versiani et al. 2013). Der Faktor 6 dient dazu, ganzzahlige Werte als Ergebnis zu erhalten (Hildebrand und Rüegsegger 1997a). Die ganzzahligen Werte werden dabei als arbiträre Einheit ausgedrückt (Kim et al. 2013).

Der Wert SMI = 0 entspricht der Struktur einer idealen plattenförmigen Fläche, $\mathrm{SMI}=3$ einer idealen zylindrischen Stabstruktur. SMI $=4$ entspricht einem perfekten Ball im dreidimensionalen Raum (You et al. 2011). Die entstandenen Werte sind dabei unabhängig von jedweder physikalischen Abmessung (Hildebrand und Rüegsegger 1997a). Der Wert des zu untersuchenden Objekts variiert so zwischen 0-4 und nimmt dabei je nach Wert eine gemischte Struktur zwischen platten- und stabartigen Konstrukten an (Hildebrand und Rüegsegger 1997b). Der SMI wird durch kleinste Veränderungen der Oberfläche bestimmt, bei der die Volumenänderung einer Wurzelkanalpräparation auf der Oberflächenund Konvexitätsänderung beruht (Peters et al. 2000). Bei einer Vergrößerung einer perfekten kreisförmigen Fläche wird die Oberfläche nicht vergrößert, da es einem SMI von 0 entspricht. Wenn eine Zylinderform vergrößert wird, steigt die Oberfläche mit dem Volumen an. Der SMI beträgt bei perfekten Zylindern 3. Der SMI kann die „Rundheit“ des Wurzelkanals quantifizieren - je höher der Wert für den SMI ist, desto runder ist auch der Wurzelkanal (Moore et al. 2009). 
In der Software CT-Analyser wurden die Werte wie bei der Volumenbestimmung vorbereitet. Mit 3D-analysis wurde der Unterpunkt „SMI“ ausgewählt und berechnet. Der SMI wurde für den prä- und postoperativen Wurzelkanal bestimmt. Die Differenz aus beiden Werten wurde als $\Delta \mathrm{SMI}$ angegeben.

\subsubsection{Canal Transportation}

Die Berechnung der Canal Transportation (CT) wurde erstmals durch Gambill et al. (1996) beschrieben und bezeichnet Abweichungen der postoperativen von der präoperativen Wurzelkanalachse nach distal oder mesial.

Zur Berechnung der CT wurden vier Wurzelquerschnitte ausgehend von den präund postoperativen Mikro-CT-Scans verwendet. Diese befanden sich jeweils 1 $\mathrm{mm}, 3 \mathrm{~mm}, 5 \mathrm{~mm}$ und $7 \mathrm{~mm}$ koronal des Apex. Für die Berechnung wurde ausgehend von den mesialen und distalen Wurzelkanalwänden beider Wurzelkanäle der geringste Abstand zur äußeren Dentinwand gemessen. Die Berechnung erfolgte nach der Formel von Gambill et al. (1996):

\section{Canal Transportation $=\left(x_{1}-x_{2}\right)-\left(y_{1}-y_{2}\right)$}

Hierbei entspricht $x_{1}$ dem geringsten Abstand von mesialer Wurzelkanalwand zur Dentinaußenfläche präoperativ. $x_{2}$ entspricht dem gleichen Abstand postoperativ. $\mathrm{y}_{1}$ entspricht dem geringsten Abstand von distaler Wurzelkanalwand zur Dentinaußenfläche präoperativ. $y_{2}$ entspricht dem gleichen Abstand postoperativ (Abb. 20). Bei einem errechneten Wert von 0 liegt keine Kanalverlagerung vor. Hierbei ist die Relation von mesialem oder distalem Abstand von Wurzelkanalwand zur äußeren Dentinwand prä- und postoperativ gleich und eine Abweichung des Wurzelkanals von der ursprünglichen Achse liegt nicht vor. Bei Werten $>0$ liegt eine mesiale und bei Werten $<0$ postoperativ eine distale Verschiebung der ursprünglichen Wurzelkanalachse vor.

Die Querschnittsbilder wurden mit der Software CT-Analyser zunächst angezeigt. Dafür wurden anhand des dreidimensional registrierten postoperativen Datensatzes die zuvor als Landmarken festgelegten Querschnitte ausgewählt. Dem gegenübergestellt wurden die gleichen Querschnitte am präoperativen Datensatz angezeigt. Mithilfe des Befehls measure wurde der geringste Abstand zwischen Wurzelkanalwand und Dentinaußenfläche in Millimetern gemessen.

Für jeden Zahn wurden insgesamt acht Abstandsmessungen durchgeführt. Für jeden Kanal wurden die vier Variablen $\mathrm{x}_{1}, \mathrm{x}_{2}, \mathrm{y}_{1}, \mathrm{y}_{2}$ ermittelt. Die Werte wurden in 
dem Programm Excel Version 15.28 (Microsoft, Redmond, USA) aufgetragen und mit der oben genannten Formel ausgerechnet.

Eine zusätzliche Visualisierung der Querschnittsbilder erfolgte mit der Software DataViewer. Die Querschnitte des Wurzelkanals bei $1 \mathrm{~mm}, 3 \mathrm{~mm}, 5 \mathrm{~mm}$ und 7 $\mathrm{mm}$ vom Apex entfernt wurden zusätzlich mit den farbig markierten prä- und postoperativen Wurzelkanalverläufen überlagert und dargestellt (Abb. 19).
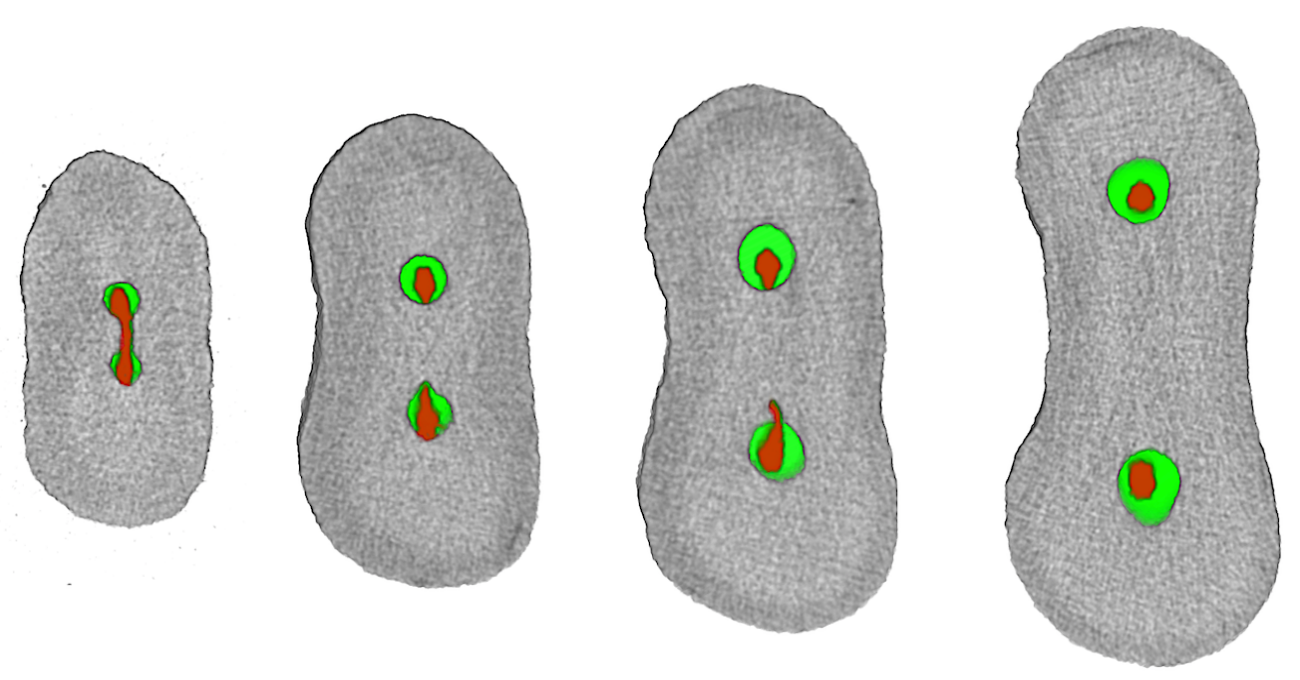

Abb. 19: Wurzelkanalquerschnitte bei $1 \mathrm{~mm}, 3 \mathrm{~mm}, 5 \mathrm{~mm}, 7 \mathrm{~mm}$ vom Apex entfernt (von links nach rechts); rot: präoperativer Wurzelkanalverlauf; grün: postoperativer Wurzelkanalverlauf

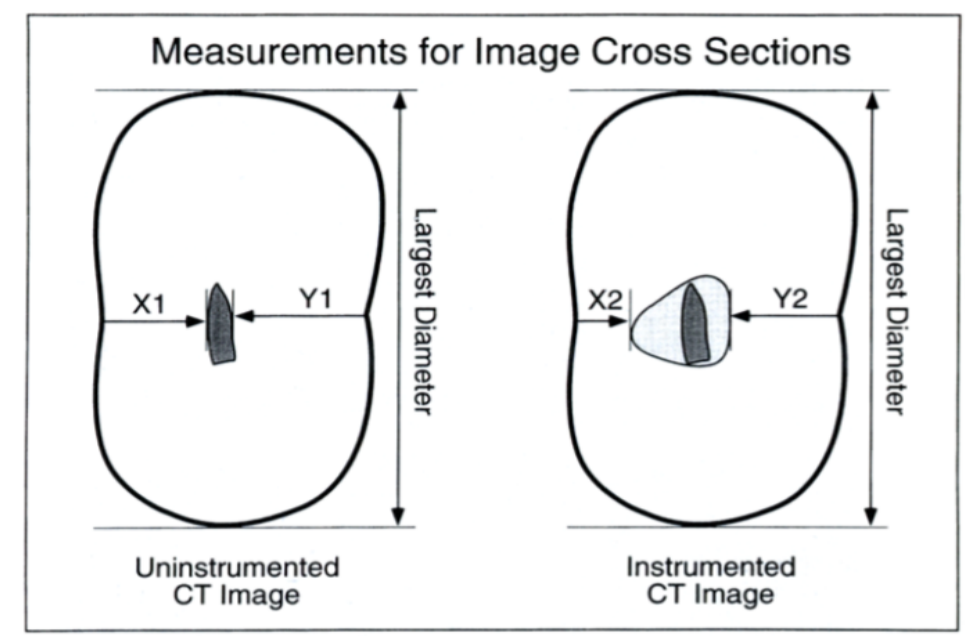

Abb. 20: Messung der Canal Transportation nach Gambill et al. (1996); X1 = geringster Abstand von mesialer Wurzelkanalwand zur äußeren Dentinfläche präoperativ; Y1 = geringster Abstand von distaler Wurzelkanalwand zur äußeren Dentinfläche präoperativ; $\mathrm{X} 2$ = geringster Abstand von mesialer Wurzelkanalwand zur äußeren Dentinfläche postoperativ; Y2 = geringster Abstand von distaler Wurzelkanalwand zur äußeren Dentinfläche postoperativ; aus Gambill et al. (1996). Die Verwendung erfolgt mit freundlicher Genehmigung von Elsevier. 


\subsubsection{Centering Ability}

Die Centering Ability (CA) wird als „die Fähigkeit des Instruments, im Kanal zentriert zu bleiben" beschrieben (Gambill et al. 1996).

Zur Berechnung der CA wurden die bereits in Kapitel 2.5.6 beschriebenen Werte der Abstandsberechnung an den Wurzelkanalquerschnitten der CT verwendet.

Die in Kapitel 2.5.6 genannte Formel von Gambill et al. (1996) wurde wie folgt zur Berechnung der CA umgestellt:

$$
\text { Centering Ability }=\frac{\left(x_{1}-x_{2}\right)}{\left(y_{1}-y_{2}\right)} \text { oder } \frac{\left(y_{1}-y_{2}\right)}{\left(x_{1}-x_{2}\right)}
$$

Hierbei entspricht $\mathrm{x}_{1}$ dem geringsten Abstand von mesialer Wurzelkanalwand zur Dentinaußenfläche präoperativ. $x_{2}$ entspricht dem gleichen Abstand postoperativ. $\mathrm{y}_{1}$ entspricht dem geringsten Abstand von distaler Wurzelkanalwand zur Dentinaußenfläche präoperativ. $y_{2}$ entspricht dem gleichen Abstand postoperativ (Abb. 20).

Die optimale Zentrierung der Präparierfeile ist bei einem Wert von 1 erreicht. Je näher die errechneten Werte am Wert 1 liegen, umso besser verbleibt das Wurzelkanalinstrument im Kanal. Je mehr die Ergebnisse sich dem Wert 0 nähern, umso weniger zentriert erfolgte die Wurzelkanalpräparation (de Carvalho et al. 2015). Die gemessenen Zahlenwerte wurden im Programm Excel Version 15.28 (Microsoft, Redmond, USA) eingetragen und die oben genannte Formel angewendet.

In der Berechnung wurden zunächst die beiden Formeln $\left(x_{1}-x_{2}\right)$ und $\left(y_{1}-y_{2}\right)$ berechnet. Die größere Summe wurde in der weiteren Rechnung als Zähler im Bruch verwendet, sodass im Ergebnis niemals ein Wert $>1$ erreicht werden konnte. 


\section{Ergebnisse}

Auf Basis der Null-Hypothese, dass sich alle drei Feilengruppen in Bezug auf die zu vergleichenden Parameter nicht unterscheiden, wurden alle Gruppen gegeneinander statistisch getestet. Jede Feilengruppe beinhaltete Versuchszähne mit einfacher und doppelter Krümmung, die als Untergruppierungen verwendet wurden.

Nach explorativer Datenanalyse mithilfe von Q-Q-Diagrammen wurde eine Normalverteilung der Werte angenommen. Die statistische Analyse wurde mit einer zweifaktoriellen Varianzanalyse (ANOVA) bei einem Signifikanzniveau von $5 \%$ durchgeführt. Hierbei wurden die statistisch signifikanten Unterschiede der Werte zwischen den Gruppen mit doppelt und einfach gekrümmten Wurzelkanälen mit den Faktoren „Gruppe/Krümmung“ berechnet.

Die Canal Transportation und Centering Ability wurden aufgrund der drei Variablen „Gruppe/Krümmung/Slice“ mit einer dreifaktoriellen Varianzanalyse (ANOVA) untersucht.

Bei allen durchgeführten Tests ergaben sich für keinen der untersuchten Parameter signifikante Unterschiede zwischen doppelten und einfachen Krümmungen (Tab. 6, Tab. 7). Die Versuchszähne einer Feilengruppe wurden daher zusammengefasst und die Untergliederung in einfach und doppelt gekrümmte Wurzelkanäle im Weiteren nicht mehr berücksichtigt.

Bei statistisch signifikanten Unterschieden der ANOVA erfolgten anschließende Post-Hoc-Tests (Tukey-Test, Scheffé-Test) zur weiteren Untersuchung.

\subsection{Veränderungen der Oberfläche}

Gemessen wurden die präoperative Fläche der Wurzelkanalwand, die Oberfläche nach erfolgter Präparation sowie die Oberflächenveränderung. Die errechneten Werte für die Oberfläche wurden sowohl für den mesiobukkalen als auch für den mesiolingualen Kanal als zusammengefasster Wert angegeben, da somit die Oberfläche der Isthmen zwischen den Kanälen miteinbezogen werden konnte. Die Ausgangswerte der Oberflächen sind in Tab. 3 zusammengefasst.

Die Oberflächenveränderung, im Weiteren als $\Delta$ Oberfläche bezeichnet, fiel für WOG mit $39.03 \pm 23.55 \%$ am geringsten aus. Der größte Zuwachs der Oberfläche fand sich für S1 Plus mit $43.42 \pm 28.71 \%$. Bei der $\Delta$ Oberfläche ergab die Varianzanalyse (ANOVA) keine statistisch signifikanten Unterschiede (Tab. 6). 


\subsubsection{Anteil der unpräparierten Oberfläche}

Der Anteil der unpräparierten Oberfläche ergibt sich aus der Differenz zwischen prä- und postoperativem Scan. Dabei wurden auch vorhandene Isthmen zwischen den beiden mesialen Wurzelkanälen in die Berechnung einbezogen.

Den geringsten Anteil an unpräparierter Oberfläche zeigte RECIPROC mit 14,70 $\pm 7,81 \%$. Bei WOG blieb mit durchschnittlich 16,44 $\pm 8,32 \%$ der größte Anteil der Wurzelkanalwand unpräpariert. Die kleinste verbliebene unpräparierte Oberfläche wurde mit WOG mit einfacher Krümmung erreicht $\left(0,89 \mathrm{~mm}^{2}\right)$, die größte unpräparierte Fläche war für S1 Plus mit ebenfalls einfacher Krümmung $(28,75$ $\mathrm{mm}^{2}$ ) zu finden. Die statistische Auswertung zeigte in allen Vergleichen keine signifikanten Unterschiede zwischen den Gruppen (Tab. 6).

Tab. 3: Veränderungen der Wurzelkanaloberfläche und Anteil der unpräparierten Wurzelkanaloberfläche in allen Feilengruppen

\begin{tabular}{|c|c|c|c|}
\hline Feilengruppe & Wurzelkanaloberfläche & $\begin{array}{c}\text { Mean, Standardab- } \\
\text { weichung }\end{array}$ & Median \\
\hline \multirow{4}{*}{ S1 Plus } & Präoperativ [mm²] & $48,96 \pm 18,87$ & 45,45 \\
\hline & Postoperativ [mm²] & $66,92 \pm 18,83$ & 66,61 \\
\hline & $\Delta$ Oberfläche $[\%]$ & $43,42 \pm 28,71$ & 29,97 \\
\hline & $\begin{array}{c}\Delta \text { Unpräparierte Ober- } \\
\text { fläche [\%] }\end{array}$ & $16,16 \pm 9,79$ & 16,59 \\
\hline \multirow{4}{*}{ WOG } & Präoperativ [mm²] & $46,86 \pm 14,76$ & 45,34 \\
\hline & Postoperativ [mm²] & $62,89 \pm 14,86$ & 61,31 \\
\hline & $\Delta$ Oberfläche [\%] & $39,03 \pm 23,55$ & 30,41 \\
\hline & $\begin{array}{c}\Delta \text { Unpräparierte Ober- } \\
\text { fläche [\%] }\end{array}$ & $16,44 \pm 8,32$ & 16,63 \\
\hline \multirow{4}{*}{ RECIPROC } & Präoperativ [mm²] & $46,43 \pm 12,03$ & 46,04 \\
\hline & Postoperativ [mm²] & $63,51 \pm 11,38$ & 63,83 \\
\hline & $\Delta$ Oberfläche [\%] & $40,73 \pm 21,75$ & 32,45 \\
\hline & $\begin{array}{c}\Delta \text { Unpräparierte Ober- } \\
\text { fläche }[\%]\end{array}$ & $14,70 \pm 7,81$ & 14,43 \\
\hline
\end{tabular}




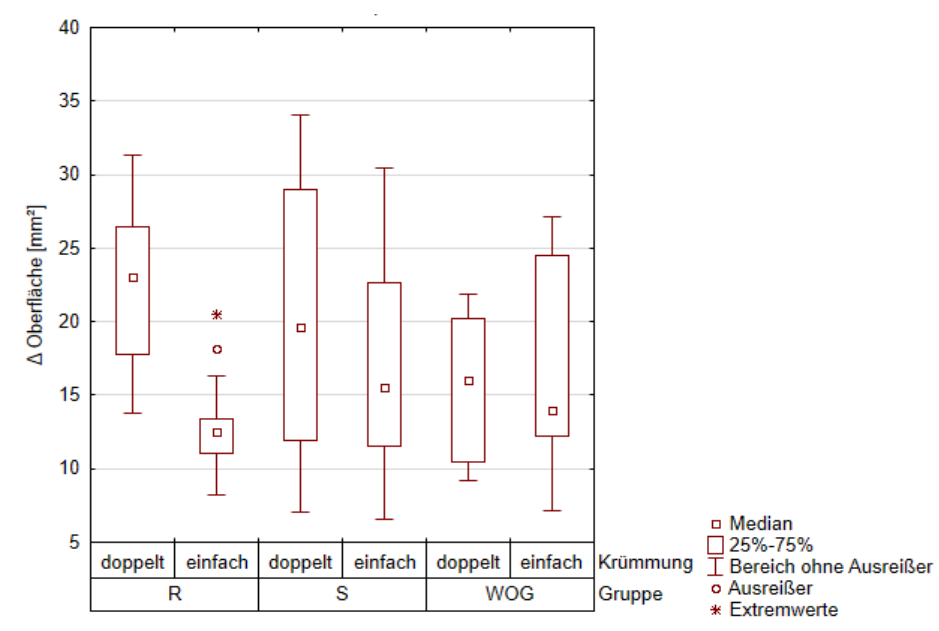

Abb. 21: Veränderung der Wurzelkanaloberfläche in den Versuchsgruppen; gemessen in $\mathrm{mm}^{2}(\mathrm{R}=\mathrm{RECIPROC}, \mathrm{S}=\mathrm{S} 1$ Plus, $W O G=$ WaveOne Gold, doppelt $=$ doppelte Krümmung, einfach $=$ einfache Krümmung)

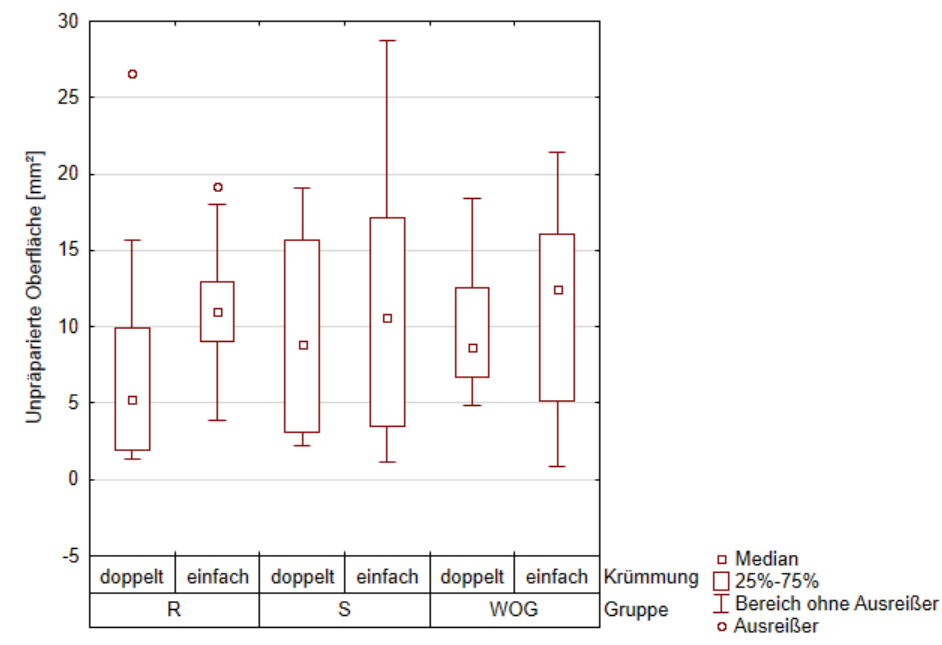

Abb. 22: Anteil der unpräparierten Wurzelkanaloberfläche in den Versuchsgruppen; gemessen in $\mathrm{mm}^{2}(\mathrm{R}=\mathrm{RECIPROC}, \mathrm{S}$ = S1 Plus, WOG = WaveOne Gold, doppelt = doppelte Krümmung, einfach = einfache Krümmung)

\subsection{Veränderung des Volumens}

Das Volumen aller Wurzelkanäle wurde durch die Wurzelkanalpräparation vergrößert. Die Differenz zwischen präoperativem und postoperativem Volumen entspricht dem aus der Präparation resultierenden Dentinabtrag. Die Werte des Volumenzuwachses ( $\Delta$ Volumen) wurden für den mesiobukkalen und mesiolingualen Kanal zusammengefasst, da eine genaue Abgrenzung der Wurzelkanäle bei vorhandenen interkanalären Isthmen nicht möglich war. 
S1 Plus zeigte den durchschnittlich größten Volumenzuwachs von 216,19 \pm 165,37 \%. Den geringsten Abtrag mit 197,32 \pm 124,73 \% zeigte WOG (Tab. 4). Die statistische Auswertung ergab in allen Vergleichen keine signifikanten Unterschiede zwischen den Gruppen und Krümmungen (Tab. 6).

Tab. 4: Veränderungen des Wurzelkanalvolumens in allen Feilengruppen

\begin{tabular}{|c|c|c|c|}
\hline Feilengruppe & Wurzelkanalvolumen & $\begin{array}{c}\text { Mean, Standardab- } \\
\text { weichung }\end{array}$ & Median \\
\hline \multirow{3}{*}{ S1 Plus } & Präoperativ [mm³] & $3,42 \pm 2,41$ & 2,37 \\
\hline & Postoperativ [mm³] & $8,59 \pm 2,92$ & 8,64 \\
\hline & $\Delta$ Volumen $[\%]$ & $216,19 \pm 165,37$ & 151,55 \\
\hline \multirow{3}{*}{ WOG } & Präoperativ [mm³] & $3,28 \pm 1,41$ & 3,44 \\
\hline & Postoperativ $\left[\mathrm{mm}^{3}\right]$ & $8,50 \pm 2,15$ & 8,58 \\
\hline & $\Delta$ Volumen [\%] & $197,32 \pm 124,73$ & 181,85 \\
\hline \multirow{3}{*}{ RECIPROC } & Präoperativ [mm³] & $3,33 \pm 1,57$ & 2,90 \\
\hline & Postoperativ [mm³] & $8,69 \pm 1,89$ & 8,67 \\
\hline & $\Delta$ Volumen $[\%]$ & $197,40 \pm 104,05$ & 188,66 \\
\hline
\end{tabular}

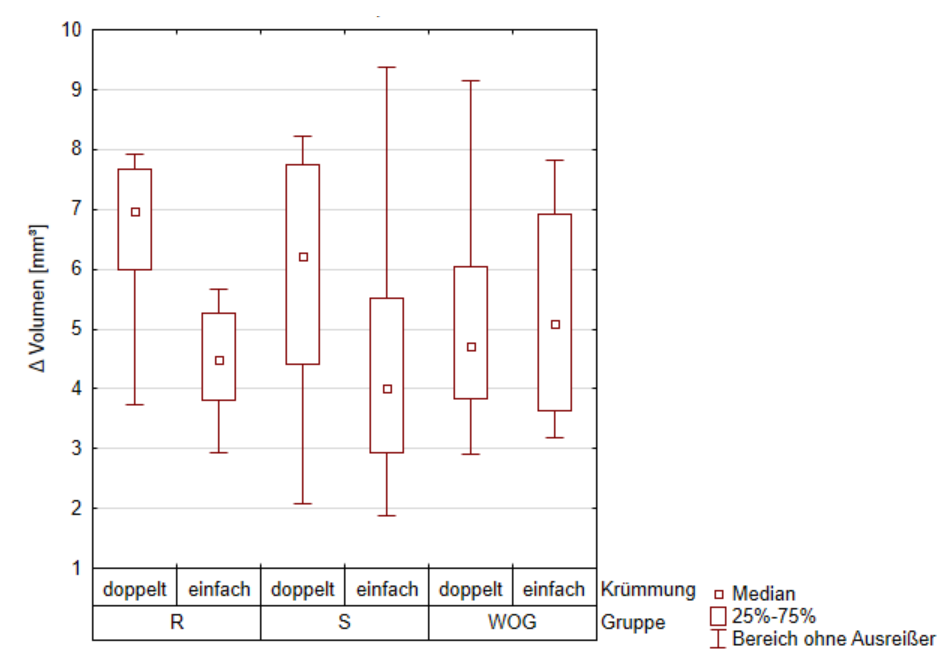

Abb. 23: Volumenveränderung der Wurzelkanäle in den Versuchsgruppen; gemessen in $\mathrm{mm}^{3}(\mathrm{R}=\mathrm{RECIPROC}, \mathrm{S}=\mathrm{S} 1$ Plus, $W O G=$ WaveOne Gold, doppelt $=$ doppelte Krümmung, einfach = einfache Krümmung) 


\subsection{Veränderung des Structure Model Index}

Die Berechnung des SMI erfolgte wie in Kapitel 2.5.5 beschrieben. Bei der Wurzelkanalpräparation wurde eine möglichst gleichmäßige Präparation angestrebt, welche durch den SMI über die Berechnung von Strukturen im dreidimensionalen Raum taxiert werden konnte.

Durchschnittlich zeigten sich ähnliche postoperative Werte des SMI in allen drei Gruppen. WOG zeigte mit 2,59 \pm 0,49 den größten Wert, gefolgt von RECIPROC mit 2,54 $\pm 0,48$ und S1 Plus mit 2,52 $\pm 0,65$. Die Änderung des SMI durch die Wurzelkanalpräparation ( $\Delta \mathrm{SMI}$ ) zeigte nur geringe Unterschiede. Ebenso wie bei den postoperativen Werten zeigte sich für WOG der größte $\Delta$ SMI mit 40,17 \pm $18,17 \%$. Die mehrfaktorielle Varianzanalyse zeigte keine statistisch signifikanten Unterschiede zwischen den Gruppen (Tab. 5-6).

Tab. 5: Veränderungen des Structure Model Index in allen Feilengruppen

\begin{tabular}{|c|c|c|c|}
\hline Feilengruppe & $\begin{array}{l}\text { Structure Model In- } \\
\text { dex }\end{array}$ & $\begin{array}{c}\text { Mean, Stan- } \\
\text { dardabweichung }\end{array}$ & Median \\
\hline \multirow{4}{*}{ S1 Plus } & Präoperativ & $1,86 \pm 0,56$ & 1,82 \\
\hline & Postoperativ & $2,52 \pm 0,65$ & 2,53 \\
\hline & $\Delta \mathrm{SMI}$ & $0,66 \pm 0,26$ & 0,69 \\
\hline & $\Delta \mathrm{SMI}[\%]$ & $38,65 \pm 19,09$ & 29,64 \\
\hline \multirow{4}{*}{ WOG } & Präoperativ & $1,90 \pm 0,51$ & 1,88 \\
\hline & Postoperativ & $2,59 \pm 0,49$ & 2,72 \\
\hline & $\Delta \mathrm{SMI}$ & $0,69 \pm 0,26$ & 0,73 \\
\hline & $\Delta \mathrm{SMI}[\%]$ & $40,17 \pm 18,17$ & 32,14 \\
\hline \multirow{4}{*}{ RECIPROC } & Präoperativ & $1,88 \pm 0,34$ & 1,95 \\
\hline & Postoperativ & $2,54 \pm 0,48$ & 2,55 \\
\hline & $\Delta \mathrm{SMI}$ & $0,65 \pm 0,28$ & 0,59 \\
\hline & $\Delta \mathrm{SMI}[\%]$ & $35,56 \pm 15,59$ & 27,62 \\
\hline
\end{tabular}


Tab. 6: p-Werte der zweifaktoriellen ANOVA

\begin{tabular}{|c|c|c|}
\hline p-Werte & Gruppe & Krümmung \\
\hline Oberfläche präoperativ & 0,81 & 0,23 \\
\hline Oberfläche postoperativ & 0,62 & 0,86 \\
\hline Unpräparierte Oberfläche & 0,71 & 0,12 \\
\hline Unpräparierte Oberfläche & 0,65 & 0,11 \\
\hline Volumen präoperativ & 0,97 & 0,13 \\
\hline Volumen postoperativ & 0,90 & 0,38 \\
\hline$\Delta$ Volumen & 0,82 & 0,15 \\
\hline$\Delta$ Structure Model Index & 0,55 & 0,97 \\
\hline
\end{tabular}

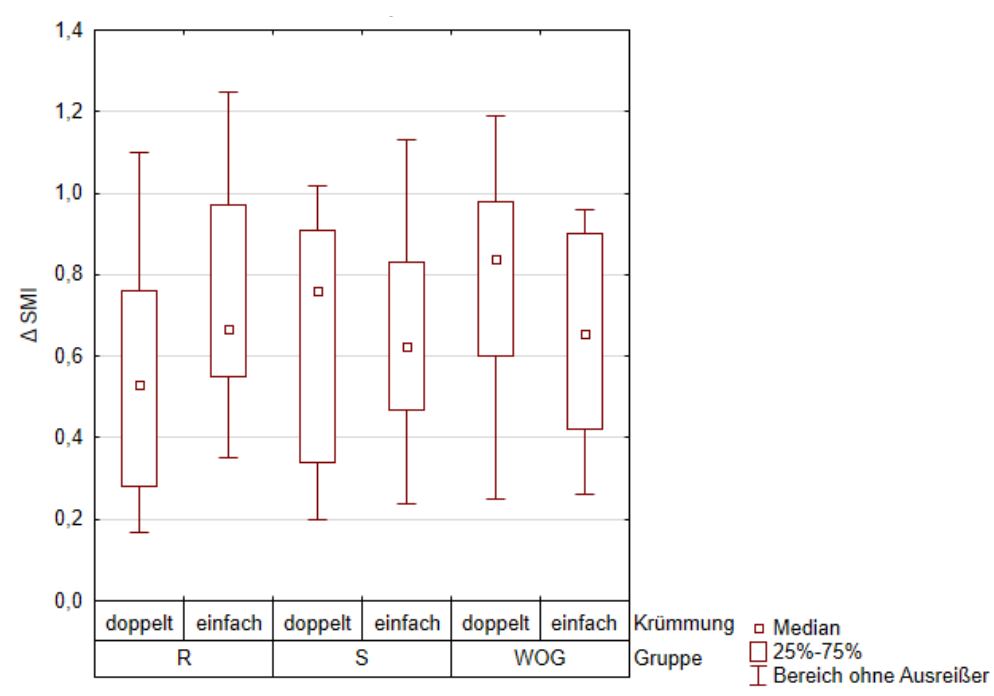

Abb. 24: Veränderung des $S M I$ in den Versuchsgruppen $(R=$ RECIPROC, $S=S 1$ Plus, $W O G=$ WaveOne Gold, doppelt = doppelte Krümmung, einfach = einfache Krümmung) 


\subsection{Veränderung der Canal Transportation}

Die Berechnung der CT wurde in Kapitel 2.5.6 erklärt. Ein Wert von 0 entspricht keiner Verlagerung des Wurzelkanals und somit einer gleichmäßigen Erweiterung durch die Präparation. Werte $>0$ entsprechen einer mesialen Verlagerung, Werte $<0$ einer distalen Verlagerung.

Die Messungen wurden an vier verschiedenen Querschnitten der Wurzelkanäle durchgeführt und statistisch durch eine dreifaktorielle Varianzanalyse (ANOVA) gegenübergestellt. Bei der Berechnung wurden die Faktoren „Gruppe/ Krümmung/slice" verwendet. Es bestand hierbei keine statistisch signifikante Interaktion $(p>0,059)$.

Die Tests der Faktoren Gruppe $(p=0,01)$ und slice $(p=0,01)$ zeigten jedoch signifikante Unterschiede, sodass weiterführende Tests durchgeführt wurden. Um zu überprüfen, welcher Post-Hoc-Test geeignet ist, wurde der Levene-Test auf Varianzhomogenität angewandt:

Im Hinblick auf den Faktor Gruppe zeigt sich ein Wert von $p=0,19$. Eine Gleichheit der Varianzen konnte angenommen werden, sodass ein Tukey-Test verwendet wurde, der keine signifikanten Unterschiede feststellte (Tab. 7).

Im Hinblick auf den Faktor slice zeigte sich weiterhin ein Wert von $p=0,01$. Nach Levene kann eine Gleichheit der Varianzen nicht angenommen werden, sodass weiterführend ein Scheffé-Test verwendet wurde. In allen durchgeführten Scheffé-Tests konnte aufgezeigt werden, dass in allen Gruppen eine signifikante Erhöhung der CT von apikal nach koronal entsteht (Tab. 9-Tab. 12). Die Ergebnisse wurden ohne Vorzeichen errechnet, um die absolute CT, unabhängig von der mesialen oder distalen Richtung, zu ermitteln.

RECIPROC zeigt mit mittelwertig $0,08 \mathrm{~mm}$ die größte CT. S1 Plus und WaveOneGold zeigen jeweils eine CT von 0,07 mm ( Tab. 13). In allen Gruppen fand sich eine deutlich zunehmende distalwärts gerichtete CT von apikal nach koronal (Tab. 14).

Tab. 7: p-Werte der dreifaktoriellen ANOVA mit den Faktoren Gruppe/Krümmung/Slice; Signifikanzen sind farbig markiert

\begin{tabular}{|c|c|c|c|}
\hline p-Werte & Gruppe & Krümmung & Slice \\
\hline Canal Transportation & 0,03 & 0,29 & 0,01 \\
\hline Centering Ability & 0,43 & 0,07 & 0,28 \\
\hline
\end{tabular}


Tab. 8: p-Werte des Tukey-Tests zwischen den Feilengruppen

\begin{tabular}{|c|c|c|c|}
\hline- & S1 Plus & WOG & RECIPROC \\
\hline S1 Plus & - & 0,97 & 0,10 \\
\hline WOG & 0,97 & - & 0,06 \\
\hline RECIPROC & 0,10 & 0,06 & - \\
\hline
\end{tabular}

Tab. 9: p-Werte des Scheffé-Tests ohne Abhängigkeit von der Feilengruppen in allen Querschnitten; Signifikanzen sind farbig markiert

\begin{tabular}{|c|c|c|c|c|}
\hline Slice & $1 \mathrm{~mm}$ & $3 \mathrm{~mm}$ & $5 \mathrm{~mm}$ & $7 \mathrm{~mm}$ \\
\hline $1 \mathrm{~mm}$ & - & 0,04 & 0,01 & 0,01 \\
\hline $3 \mathrm{~mm}$ & 0,04 & - & 0,90 & 0,01 \\
\hline $5 \mathrm{~mm}$ & 0,01 & 0,90 & - & 0,01 \\
\hline $7 \mathrm{~mm}$ & 0,01 & 0,01 & 0,01 & - \\
\hline
\end{tabular}

Tab. 10: p-Werte des Scheffé-Tests innerhalb der Gruppe S1 Plus in allen Querschnitten; Signifikanzen sind farbig markiert

\begin{tabular}{|c|c|c|c|c|}
\hline Slice & $1 \mathrm{~mm}$ & $3 \mathrm{~mm}$ & $5 \mathrm{~mm}$ & $7 \mathrm{~mm}$ \\
\hline $1 \mathrm{~mm}$ & - & 0,85 & 0,47 & 0,01 \\
\hline $3 \mathrm{~mm}$ & 0,85 & - & 0,92 & 0,07 \\
\hline $5 \mathrm{~mm}$ & 0,47 & 0,92 & - & 0,27 \\
\hline $7 \mathrm{~mm}$ & 0,01 & 0,07 & 0,27 & - \\
\hline
\end{tabular}


Tab. 11: p-Werte des Scheffé-Tests innerhalb der Gruppe WOG in allen Querschnitten; Signifikanzen sind farbig markiert

\begin{tabular}{|c|c|c|c|c|}
\hline Slice & $1 \mathrm{~mm}$ & $3 \mathrm{~mm}$ & $5 \mathrm{~mm}$ & $7 \mathrm{~mm}$ \\
\hline $1 \mathrm{~mm}$ & - & 0,22 & 0,10 & 0,01 \\
\hline $3 \mathrm{~mm}$ & 0,22 & - & 0,98 & 0,01 \\
\hline $5 \mathrm{~mm}$ & 0,10 & 0,98 & - & 0,01 \\
\hline $7 \mathrm{~mm}$ & 0,01 & 0,01 & 0,01 & - \\
\hline
\end{tabular}

Tab. 12: p-Werte des Scheffé-Tests innerhalb der Gruppe RECIPROC in allen Querschnitten; Signifikanzen sind farbig markiert

\begin{tabular}{|c|c|c|c|c|}
\hline Slice & $1 \mathrm{~mm}$ & $3 \mathrm{~mm}$ & $5 \mathrm{~mm}$ & $7 \mathrm{~mm}$ \\
\hline $1 \mathrm{~mm}$ & - & 0,22 & 0,14 & 0,01 \\
\hline $3 \mathrm{~mm}$ & 0,22 & - & 0,10 & 0,01 \\
\hline $5 \mathrm{~mm}$ & 0,14 & 0,10 & - & 0,01 \\
\hline $7 \mathrm{~mm}$ & 0,01 & 0,01 & 0,01 & - \\
\hline
\end{tabular}

Tab. 13: Mittelwerte der Canal Transportation ohne Berücksichtigung der Mesial- oder Distalverlagerung und Unterscheidung in mesiobukkalen und mesiolingualen Wurzelkanal

\begin{tabular}{|c|c|c|c|c|}
\hline Slice & $1 \mathrm{~mm}$ & $3 \mathrm{~mm}$ & $5 \mathrm{~mm}$ & $7 \mathrm{~mm}$ \\
\hline Alle Gruppen & $0,04 \pm 0,04$ & $0,06 \pm 0,04$ & $0,07 \pm 0,05$ & $0,11 \pm 0,08$ \\
\hline S1 Plus & $0,05 \pm 0,06$ & $0,06 \pm 0,04$ & $0,07 \pm 0,05$ & $0,09 \pm 0,08$ \\
\hline WOG & $0,03 \pm 0,03$ & $0,06 \pm 0,04$ & $0,07 \pm 0,05$ & $0,11 \pm 0,08$ \\
\hline RECIPROC & $0,05 \pm 0,04$ & $0,07 \pm 0,05$ & $0,08 \pm 0,06$ & $0,13 \pm 0,09$ \\
\hline
\end{tabular}

Mittelwertangaben in Millimetern und mit Standardabweichung $(\mathrm{mb}=$ mesiobukkaler Kanal, $\mathrm{ml}=$ mesiolingualer Kanal) 
Tab. 14: Mittelwerte der Canal Transportation mit Berücksichtigung der Mesial- und Distalverlagerung aller vier Querschnitte

\begin{tabular}{|c|c|c|c|c|c|}
\hline \multicolumn{2}{|c|}{ Gruppe } & $1 \mathrm{~mm}$ & $3 \mathrm{~mm}$ & $5 \mathrm{~mm}$ & $7 \mathrm{~mm}$ \\
\hline \multirow{2}{*}{ Alle Gruppen } & $\mathrm{mb}$ & $0,03 \pm 0,05$ & $0,03 \pm 0,07$ & $-0,02 \pm 0,08$ & $-0,1 \pm 0,23$ \\
\hline & $\mathrm{ml}$ & $0,02 \pm 0,07$ & $0,02 \pm 0,07$ & $-0,02 \pm 0,09$ & $-0,09 \pm 0,12$ \\
\hline \multirow{2}{*}{ S1 Plus } & $\mathrm{mb}$ & $0,02 \pm 0,06$ & $0,00 \pm 0,06$ & $-0,04 \pm 0,07$ & $-0,09 \pm 0,06$ \\
\hline & $\mathrm{ml}$ & $0,00 \pm 0,09$ & $0,05 \pm 0,07$ & $-0,02 \pm 0,1$ & $-0,08 \pm 0,12$ \\
\hline \multirow{2}{*}{ WOG } & $\mathrm{mb}$ & $0,02 \pm 0,04$ & $0,04 \pm 0,08$ & $0,0 \pm 0,08$ & $-0,09 \pm 0,1$ \\
\hline & $\mathrm{ml}$ & $0,02 \pm 0,05$ & $0,0 \pm 0,06$ & $-0,02 \pm 0,08$ & $-0,08 \pm 0,11$ \\
\hline \multirow{2}{*}{ RECIPROC } & $\mathrm{mb}$ & $0,04 \pm 0,05$ & $0,06 \pm 0,07$ & $-0,01 \pm 0,09$ & $-0,12 \pm 0,07$ \\
\hline & $\mathrm{ml}$ & $0,03 \pm 0,07$ & $0,0 \pm 0,09$ & $-0,02 \pm 0,1$ & $-0,1 \pm 0,14$ \\
\hline
\end{tabular}

Mittelwertangaben in Millimetern und mit Standardabweichung $(\mathrm{mb}=$ mesiobukkaler Kanal, $\mathrm{ml}=$ mesiolingualer Kanal)

\subsection{Veränderung der Centering Ability}

Die CA beschreibt das Verhalten und die Fähigkeit der Wurzelkanalfeile, zentriert im Wurzelkanal zu bleiben. Die Berechnung der CA wurde in Kapitel 2.5.7 erklärt. Der Wert 1 entspricht einer perfekten Zentrierung des Instruments im Wurzelkanal. Je näher die Messwerte am Wert 1 liegen, umso besser verbleibt das Instrument zentriert im Wurzelkanal. Werte über 1 sind nicht möglich.

Die höchste mittlere CA lag mittelwertig für WOG mit $0,65 \pm 0,24$ vor $R$ mit 0,63 $\pm 0,23$ und S1 Plus mit 0,60 $\pm 0,25$ (Tab. 15). Es lag in allen Gruppen keine Veränderung der $\mathrm{CA}$ vor.

Bei der dreifaktoriellen ANOVA wurden mit den Faktoren "Gruppe*Krümmung ${ }^{*}$ slice" keine statistisch signifikanten Unterschiede festgestellt. Es ergaben sich bei den einzelnen Faktoren folgende $p$-Werte:

Gruppe: $p=0,44$, Krümmung: $p=0,07$, Slice: $p=0,28$.

Bei $p$-Werten von $p>0,05$ wurden keine weiterführenden Post-Hoc-Tests durchgeführt. 
Tab. 15: Mittelwerte der Centering Ability mit Berücksichtigung der Mesial- und Distalverlagerung aller vier Querschnitte

\begin{tabular}{|c|c|c|c|c|c|}
\hline \multicolumn{2}{|c|}{ Slice } & $1 \mathrm{~mm}$ & $3 \mathrm{~mm}$ & $5 \mathrm{~mm}$ & $7 \mathrm{~mm}$ \\
\hline \multicolumn{2}{|l|}{ S1 Plus } & $0,58 \pm 0,27$ & $0,59 \pm 0,23$ & $0,64 \pm 0,23$ & $0,6 \pm 0,24$ \\
\hline \multicolumn{2}{|l|}{ WOG } & $0,7 \pm 0,26$ & $0,62 \pm 0,25$ & $0,67 \pm 0,22$ & $0,62 \pm 0,22$ \\
\hline \multicolumn{2}{|c|}{ RECIPROC } & $0,63 \pm 0,28$ & $0,63 \pm 0,21$ & $0,65 \pm 0,23$ & $0,61 \pm 0,21$ \\
\hline \multirow{2}{*}{ Alle Gruppen } & $\mathrm{mb}$ & $0,65 \pm 0,27$ & $0,64 \pm 0,21$ & $0,69 \pm 0,24$ & $0,62 \pm 0,22$ \\
\hline & $\mathrm{ml}$ & $0,59 \pm 0,26$ & $0,59 \pm 0,24$ & $0,63 \pm 0,23$ & $0,60 \pm 0,23$ \\
\hline \multirow{2}{*}{ S1 Plus } & $\mathrm{mb}$ & $0,6 \pm 0,3$ & $0,66 \pm 0,21$ & $0,7 \pm 0,27$ & $0,61 \pm 0,21$ \\
\hline & $\mathrm{ml}$ & $0,56 \pm 0,23$ & $0,53 \pm 0,24$ & $0,59 \pm 0,24$ & $0,60 \pm 0,26$ \\
\hline \multirow{2}{*}{ WOG } & $\mathrm{mb}$ & $0,72 \pm 0,22$ & $0,61 \pm 0,21$ & $0,71 \pm 0,21$ & $0,65 \pm 0,21$ \\
\hline & $\mathrm{ml}$ & $0,58 \pm 0,30$ & $0,63 \pm 0,28$ & $0,64 \pm 0,23$ & $0,58 \pm 0,23$ \\
\hline \multirow{2}{*}{ RECIPROC } & $\mathrm{mb}$ & $0,63 \pm 0,30$ & $0,65 \pm 0,23$ & $0,65 \pm 0,23$ & $0,60 \pm 0,21$ \\
\hline & $\mathrm{ml}$ & $0,62 \pm 0,25$ & $0,61 \pm 0,18$ & $0,65 \pm 0,22$ & $0,62 \pm 0,21$ \\
\hline
\end{tabular}

Mittelwertangaben in Millimetern und mit Standardabweichung $(\mathrm{mb}=$ mesiobukkaler Kanal, $\mathrm{ml}=$ mesiolingualer Kanal)

\subsection{Arbeitssicherheit}

Während der Präparation der 75 Versuchszähne mit 150 Wurzelkanälen traten keine Instrumentenfrakturen auf. Bei Versuchszahn 5 mit einer doppelten Krümmung aus der Gruppe S1 Plus wurde eine starke Deformation der Feile beobachtet, worauf diese ausgetauscht wurde. Bei allen Versuchszähnen konnte die Arbeitslänge erreicht und die Präparation abgeschlossen werden. Es entstanden keine Stufen oder Perforationen. 


\section{Diskussion}

\subsection{Diskussion der Methode}

Ziel der vorliegenden Ex-vivo-Studie war der Vergleich von drei NiTi-Einfeilensystemen mit reziproker Arbeitsweise zur Präparation von Wurzelkanälen mit einfacher und doppelter Krümmung. Der Versuchsaufbau wurde in Anlehnung an die Studien von Marceliano-Alves et al. (2015) und da Silva Limoeiro et al. (2016) erstellt. Die Untersuchungen zur Bestimmung des Volumenabtrags, der CT, der CA, des SMI, der Veränderung der Oberfläche und der Bestimmung der unpräparierten Oberfläche wurden wie vergleichbare Studien mithilfe der Mikro-CT durchgeführt (de Carvalho et al. 2015; Hildebrand und Rüegsegger 1997a; Paqué et al. 2010; Peters et al. 2000). Die Nullhypothese ging davon aus, dass die drei Feilengruppen sich im Hinblick auf die oben genannten Parameter gleich verhalten.

\subsubsection{Auswahl der Versuchszähne}

Wie in vergleichbaren Studien wurden extrahierte humane Unterkiefermolaren mit gekrümmten mesialen Wurzelkanälen verwendet (Aydin et al. 2019; Elashiry et al. 2020; Marceliano-Alves et al. 2015). Bei der Verwendung natürlicher Zähne ergibt sich aufgrund unterschiedlicher Volumina, Oberflächenabmessungen, Isthmen und wechselnden Wurzelquerschnitten eine begrenzte Vergleichbarkeit der Wurzelkanalanatomie. Zur besseren Standardisierung der Versuche wurden in vielen Studien simulierte Kanäle aus Kunststoffblöcken mit identischer Kanalanatomie verwendet (Al-Sudani et al. 2012; Bonaccorso et al. 2009; Neelakantan et al. 2016; Topçuoglu et al. 2017; Uslu et al. 2018). Die Härte des Kunstoffs ist jedoch nicht vergleichbar mit der des Dentins. Daraus resultierend zeigt sich ein verändertes Abspanverhalten bei der Verwendung einer Wurzelkanalfeile während der Präparation (Kazemi et al. 1996; Miserendino et al. 1988).

Durch Angleichung der Arbeitslänge aller Versuchszähne, Einteilung in Gruppen mit nahezu gleichen Wurzelkrümmungen und Standardisierung des Präparationsprotokolls wurde versucht, die Vergleichbarkeit so hoch wie möglich zu halten (Hülsmann et al. 2003).

Die spätere Gruppenaufteilung auf Grundlage von gleichen mittleren Krümmungswinkeln und -radien der Versuchsgruppen wurde bereits in mehreren vergleichbaren Studien vorgenommen (De-Deus et al. 2015; Hülsmann et al. 2003; Paqué et al. 2010; Rödig et al. 2012; You et al. 2011). 
Die Verwendung von Zähnen mit doppelten Krümmungen in Wurzelkanälen als Bestandteil einer Studie wurde in der Literatur hingegen bisher nur zur Detektion von Ermüdungsbrüchen in simulierten Wurzelkanälen oder in Fallberichten zur Wurzelkanalpräparation in humanen Zähnen benutzt (Al-Sudani et al. 2012; Burroughs et al. 2012; Topçuoglu et al. 2016, 2017). Es finden sich keine vergleichbaren Studien mit der Verwendung von Versuchszähnen mit doppelten Krümmungen, wie sie in dieser Studie benutzt werden.

\subsubsection{Gruppeneinteilung}

Die Einteilung der Versuchszähne in drei gleichgroße Gruppen ist häufig in vergleichbaren Studien vorzufinden (Aydin et al. 2019; Burroughs et al. 2012; Cassimiro et al. 2018; Delai et al. 2019; Duque et al. 2019; Gagliardi et al. 2015; van der Vyver et al. 2019). Dabei wurden stets homogene Gruppen mit der gleichen Anzahl an Versuchszähnen sowie vergleichbaren einfachen Wurzelkanalkrümmungen verwendet. Die Untergruppierung mit der Verwendung von Versuchszähnen mit doppelten Wurzelkanalkrümmungen wurde in dieser Studie erstmalig verwendet. Versuche mit einfacher als auch doppelter Krümmung innerhalb einer Studie wurden bisher nur mit simulierten Kanälen durchgeführt (AlObaida et al. 2019; Duke et al. 2015; Elnaghy und Elsaka 2018).

Die Gruppeneinteilung erfolge durch Bildung von Gruppen von drei Versuchszähnen mit vergleichbaren Wurzelkanalkrümmungen (matched triples). Jeder Versuchszahn aus den gebildeten Drillingen wurde randomisiert einer Feilengruppe zugeteilt. Dieser Vorgang wurde mit Ausnahme der Verwendung von unterschiedlichen Krümmungen in mehreren Studien als pair matched root canals auf Basis gleicher anatomischer Merkmale durchgeführt (Belladonna et al. 2018; DeDeus et al. 2019b; De-Deus et al. 2020; Metzger et al. 2010).

\subsubsection{Wurzelkanalpräparation}

Die verwendeten Feilen wurden gemäß den Vorgaben des Herstellers verwendet. Neben der reziproken Arbeitsweise können die leichten Auf- und Abbewegungen der Präparationsfeile die Schneidleistung verbessern (Giansiracusa Rubini et al. 2014). Durch zu geringe Aufbereitungsgrößen erhöht sich das Risiko einer unvollständigen Wurzelkanalpräparation mit erhöhtem Anteil an unpräparierten Oberflächen und somit noch verbliebendem infizierten Dentin im Wurzelkanal (Bürklein et al. 2012). Demgegenüber können zu ausgedehnte Präparationen des Wurzelkanals zu einer Schwächung der Zahnhartsubstanz führen, jedoch eine verbesserte Desinfektion gewährleisten (Hülsmann et al. 2005; 
Zandbiglari et al. 2006). Laut Saber et al. (2015) kann die Wurzelkanalpräparation mit Instrumenten über der Größe ISO 30 die Wahrscheinlichkeit einer Wurzelkanalbegradigung erhöhen.

Die Feilensysteme besitzen unterschiedliche Konizitäten von 25/.06 (S1 Plus), 25/.07 (WOG) und 25/.08 (RECIPROC). Sie weisen somit keine einheitliche Geometrie auf. Dennoch vergleichen auch weitere Studien Feilensysteme mit unterschiedlichen Konizitäten (Cassimiro et al. 2018; Duque et al. 2019; Keskin et al. 2019; Kim et al. 2013; Saleh et al. 2015). Die verglichenen Feilensysteme verfügen zudem über unterschiedliche Querschnittsprofile. Die Präparationsfeile von WOG hat einen Querschnitt eines Parallelogramms, die Präparationsfeilen von S1 Plus und RECIPROC haben einen s-förmigen Querschnitt. Derzeit liegen noch keine vergleichbaren Studien mit Verwendung der Präparationsfeile S1 Plus vor. In anderen Studien mit humanen Versuchszähnen zeigten sich bei der Verwendung von RECIPROC und WOG keine signifikanten Unterschiede, die auf die unterschiedliche Feilengeometrie zurückzuführen sind (Bürklein et al. 2019; Mendonça de Moura et al. 2019). Studien zur Bewertung von Ermüdungsbrüchen in künstlichen Wurzelkanälen weisen jedoch auf eine signifikant höhere Frakturresistenz von WOG gegenüber RECIPROC hin (Keskin et al. 2017; Özyürek 2016).

\subsubsection{Auswertung mit Mikro-CT}

Die notwendige dreidimensionale Darstellung und Berechnung der Versuchsparameter sowie die Visualisierung der komplexen Wurzelkanalsysteme erfolgte mit der Mikro-CT (Dowker et al. 1997). Neben der dreidimensionalen Analyse von Oberfläche und Volumen des Wurzelkanals kann auch die zweidimensionale Wurzelquerschnittanalyse orthogonal zur Längsachse des Wurzelkanals in mehreren Ebenen erfolgen (Alovisi et al. 2017; Alovisi et al. 2018; Pasqualini et al. 2015).

Deutliche Vorteile der Micro-CT-Auswertung liegen neben der noninvasiven Diagnostik in der Reproduzierbarkeit von Scans und Analysen sowie in der hohen Detailtreue. Mit dem benutzten Gerät ist eine Zwei-Punkt-Diskriminierung in der Größe von 0,35 $\mu \mathrm{m}$ bei höchster Vergrößerung möglich (Bruker 2018). Die Messparameter wurden für alle Scans gleich festgelegt. Die Auswahl der Stromstärke und der Voxelgröße und die Verwendung von Metallfiltern wurde in Anlehnung an andere Studien getroffen (De-Deus et al. 2015; Pasqualini et al. 2015). Leichte Variationen in den vorab festgelegten Einstellungen können aus der Benutzung verschiedener Scanner resultieren. Andere Studien wählten aufgrund 
anderer Fragestellungen auch andere Parameter aus, um beispielsweise Wurzelkanalfüllungen von der Zahnhartsubstanz besser abgrenzen zu können (Rödig et al. 2012; Rödig et al. 2018).

In vorangegangenen Studien wurde zur Versuchsdurchführung und der prä- und postoperativen Wurzelquerschnittsbewertung ein standardisiertes Muffelsystem verwendet (Hiller 2014; Hülsmann 1998). Diese Methode wurde zuerst von Bramante et al. (1987) beschrieben. Die Verwendung des Muffelsystems war aufgrund des begrenzten Platzes im Scanner der Mikro-CT von maximal $75 \mathrm{~mm}$ im Durchmesser und der Röntgenopazität der Muffel nicht möglich (Bruker 2018).

Bedingt durch die gewählte Voxelgröße in der Voreinstellung des Scanners können Artefaktfehler als sogenannter partieller Volumeneffekt in Erscheinung treten. Dabei ist die Voxelgröße zu groß und überlappt zwei unterschiedliche Medien bzw. Gewebe (Erlandsson et al. 2012). In der vorliegenden Studie betrifft dies den Übergang von Dentin zu Luft im Wurzelkanal. Die Voxel würden teilweise zum falschen Gewebe gezählt werden, wodurch in der Volumetrie das gemessene Volumen entweder zu groß oder zu klein ausfallen würde. Verschiedene Studien zeigen, dass zur Beurteilung des Wurzelkanals mit Berechnung des Volumens eine Voxelgröße von unter $40 \mu \mathrm{m}$ bis $20 \mu \mathrm{m}$ ausreichend ist (Bergmans et al. 2001; Gu et al. 2010; Peters et al. 2001b).

Bei der Rekonstruktion des Datensatzes führen geringe Fehler an der Detektorplatte des Scanners zu Ringartefakten, die durch die Drehung der zu scannenden Probe entstehen. Durch die Software NRecon konnten die Ringartefakte teilweise ausgeglichen werden, zeigten jedoch weiterhin geringe ringförmige Messfehler.

Ohne vorab festgelegte Positionen der Probe im Scanner waren deutlich unterschiedliche Positionen und Richtungen der Versuchszähne im Datensatz von prä- und postoperativem Scan zu erkennen. Eine dreidimensionale Registrierung der Datensätze zueinander war notwendig, um eine Analyse in der Volumetrie zur Differenzbestimmung durchführen zu können. Durch die Software DataViewer konnten die Scans zunächst rechnerisch durch das Programm selbst übereinander gelagert werden. Durch das postoperativ veränderte Volumen und die Veränderung der Zahnhartsubstanz war die Registrierung und Kalibrierung der Datensätze bei jedem Versuchszahn fehlerhaft. Eine visuelle Angleichung der Datensätze durch den Anwender musste durchgeführt werden. Dies führte jedoch zu einer weiteren Subjektivierung und geringeren Reproduzierbarkeit der Einstellungen. 


\subsubsection{Berechnung der Versuchsparameter}

Die Berechnung der Versuchsparameter erfolgte ausschließlich über die durch die Mikro-CT generierten Datensätze. Zur Vereinfachung der Berechnung wurden alle Datensätze in binäre Bilder umgewandelt. Eine klare Differenzierung konnte dadurch zwischen dem Medium Luft und Dentin getroffen werden. Daraus resultierend war die genaue Rekonstruktion des Wurzelkanals möglich. Nachteilig zeigten sich vor allem in den präoperativen Scans befindliche Debris im Wurzelkanal, welche fälschlicherweise im binären Bild als Dentin beziehungsweise Hartsubstanz angesehen wurden. Die Debrisartefakte konnten mithilfe der Software CT-Analyser größtenteils rechnerisch entfernt werden, führten dennoch zu geringen Abweichungen.

Die Berechnungsgrenzen des Volumens im Wurzelkanal wurden, wie in Kapitel 2.5.3 beschrieben, genau festgelegt. Anhand des Querschnittbildes der Scans wurden die Grenzen subjektiv visuell an den Datensätzen durch den Anwender festgelegt. Apikal erfolgte die Orientierung an den postoperativen Scans, in denen sich eine klare Abgrenzung von präparierter und nicht präparierter Wurzelkanaloberfläche treffen ließ.

Bei der Oberflächenbestimmung wurde der komplette Umfang der Wurzelkanaloberfläche beider Wurzelkanäle der mesialen Wurzel berücksichtigt. Dadurch wurden vorhandene Isthmen zwischen dem mesiobukkalen und mesiolingualen Wurzelkanal in die Berechnung miteingeschlossen. Durch die unterschiedlich großen interkanalären Räume wurden deutliche Unterschiede in den präoperativen Oberflächen gemessen. Der Versuch, die Isthmen und Seitenkanäle nicht in die Berechnung einfließen zu lassen, hätte eine deutliche Subjektivierung der Bewertung bedeutet und die Oberflächendifferenz ungenau wiedergegeben. Vergleichbare Studien gingen nach dem gleichen Prinzip zur Bestimmung des Volumens, der Oberfläche und dem Anteil der unpräparierten Oberfläche vor (Alovisi et al. 2017; da Silva Limoeiro et al. 2016; Versiani et al. 2013; Yang et al. 2011).

Der SMI kann die Qualität einer Präparationsform bewerten (Hildebrand und Rüegsegger 1997a). Höhere Werte des SMI geben einen runderen Wurzelkanal nach der Präparation an (Moore et al. 2009). Vergleichbare Studien verwendeten ebenfalls den SMI als zusätzliche dreidimensionale Bewertung der Wurzelkanalpräparation (Aydin et al. 2019; de Almeida et al. 2015; Paqué et al. 2009; Peters et al. 2000; Stringheta et al. 2019; Thomas et al. 2020).

Die CT und die CA wurden auf Grundlage des von Gambill et al. (1996) beschriebenen und in vielen Studien verwendeten Verfahrens berechnet (Belladonna et al. 2018; de Albuquerque et al. 2019; Drukteinis et al. 2019; Duque et al. 2019; 
Gergi et al. 2014; Perez Morales et al. 2020; Poly et al. 2019; Yamamura et al. 2012; Zanette et al. 2014).

\subsection{Diskussion der Ergebnisse}

\subsubsection{Veränderung der Oberfläche}

Die Oberfläche der Wurzelkanalwand wird durch die Präparation maßgeblich verändert und vergrößert. Mehrere Studien zeigten aber, dass stets ein nicht unerheblicher Teil der Wurzelkanaloberfläche bei der Wurzelkanalpräparation unberührt bleibt (Hübscher et al. 2003; Peters et al. 2010; Peters et al. 2001a; Peters et al. 2001b).

Die präoperativen Werte der Wurzelkanaloberfläche lagen in dieser Studie mittelwertig bei 48,96 \pm 18,87 $\mathrm{mm}^{2}$ (S1 Plus), 46,86 \pm 14,76 $\mathrm{mm}^{2}$ (WOG) und 46,43 $\pm 12,03 \mathrm{~mm}^{2}$ (RECIPROC). In vergleichbaren Studien mit der Präparation mesialer Wurzelkanäle von Unterkiefermolaren lagen die mittelwertigen Ausgangswerte der Oberfläche bei 42,95 $\pm 15,34 \mathrm{~mm}^{2}$ bis 49,78 $\pm 17,26 \mathrm{~mm}^{2}$ (Stringheta et al. 2019). Marceliano-Alves et al. (2015) und Pedullà et al. (2016) maßen hingegen deutlich geringere initiale Oberflächenwerte von $22,14 \pm 17,8 \mathrm{~mm}^{2}$ bis $25,38 \pm 19,17 \mathrm{~mm}^{2}$ sowie $17,03 \pm 4,63 \mathrm{~mm}^{2}$ bis $20,6 \pm 5,47 \mathrm{~mm}^{2}$. Eine größere region of interest wurde in beiden Studien im Vergleich zu dieser Studie nicht festgelegt, sodass von kleineren interkanalären Isthmen ausgegangen werden muss. Siqueira et al. (2013) berechneten die Wurzelkanaloberflächen beider mesialen Kanäle getrennt voneinander.

Durch die Präparation vergrößerten sich in allen Versuchsgruppen die Oberflächen der Wurzelkanäle ohne signifikante Unterschiede zwischen den Gruppen.

Die geringste Oberflächenvergrößerung fand sich bei WOG mit 39,03 $\pm 23,55 \%$ auf $62,89 \pm 14,86 \mathrm{~mm}^{2}$. Die Vergrößerungen in den anderen Gruppen veränderten sich um 40,73 $\pm 21,75 \%$ auf 63,51 \pm 11,38 $\mathrm{mm}^{2}$ (RECIPROC) und um 43,42 $\pm 28,71 \%$ auf $66,92 \pm 18,83 \mathrm{~mm}^{2}$ (S1 Plus).

Pedullà et al. (2016) gaben vergleichbare prozentuale Vergrößerungen der Oberfläche um 39,62 \pm 27,31 \% mit dem Feilensystem Mtwo (Sweden \& Martina, Padova, Italien) und um 45,95 \pm 31,02 \% mit dem Feilensystem Twisted File Adaptive (TFA) (SybronEndo, Orange, USA) in reziproker Arbeitsweise an.

Marceliano-Alves et al. (2015) gaben für die Verwendung der Feilensysteme WaveOne und RECIPROC prozentuale Vergrößerungen der Wurzelkanaloberfläche durch die Präparation von $32,2 \pm 14,1 \%$ und $51,1 \pm 24,7 \%$ an. 
Siqueira et al. (2013) berechneten die Wurzelkanaloberfläche ohne die interkanalären Isthmen. Wie zu erwarten fielen die prozentualen Vergrößerungen der Wurzelkanaloberfläche geringer aus. Kombiniert im mesiobukkalen und mesiolingualen Wurzelkanal konnte eine Vergrößerung von 10,4 \pm 8,6 \% mit dem Feilensystem RECIPROC, um 9,8 \pm 6,3\% mit der Self Adjusting File (ReDent-Nova, Ra'anana, Israel) und um 10,3 \pm 6,6 \% mit dem Feilensystem Twisted File erreicht werden. Die Werte liegen deutlich niedriger als in den anderen genannten Studien. Aufgrund der Vernachlässigung der Isthmen und Seitenkanäle in der Berechnung ist ein Vergleich zu den vorliegenden Daten und zu einer In-vivo-Wurzelkanalpräparation nicht sinnvoll.

Die Qualität der Wurzelkanalpräparation wird neben den in Kapitel 1.3 und 1.3.2 beschriebenen Faktoren ebenfalls an der postoperativ unpräpariert verbliebendem Wurzelkanaloberfläche bewertet. Dieser Aspekt hat in der klinischen Anwendung keine Relevanz, da diese aktuell klinisch nicht messbar ist. Die vorliegende Studie gibt für die prozentualen Anteile der unpräparierten Oberfläche keine signifikanten Unterschiede zwischen den Gruppen an.

Die Versuchszähne mit doppelten Wurzelkanalkrümmungen zeigten ebenfalls keine signifikanten Veränderungen. Das Feilensystem RECIPROC erzielte mit $14,70 \pm 7,81 \%$ den geringsten unpräparierten Anteil der Wurzelkanaloberfläche. S1 Plus und WOG erzielten unpräparierte Anteile von 16,16 \pm 9,79 \% und 16,44 $\pm 8,32 \%$.

Stringheta et al. (2019) ermittelten einen vergleichbaren prozentualen Anteil an unpräparierter Oberfläche von 14,43 $\pm 11,85 \%$ bei der Präparation mit RECIPROC. WOG ließ 18,96 \pm 12,45 \% der Wurzelkanaloberfläche unpräpariert, ProDesign Logic (Easy Equipamentos Odontológicos, Belo Horizonte, Brasilien) ließ mit 21,13 \pm 18,72 \% den größten Teil der Wurzelkanaloberfläche unpräpariert.

Gagliardi et al. (2015) und da Silva Limoeiro et al. (2016) erzielten teils geringere Werte an unpräparierter Oberfläche von 11,42-15,46 \% und 6-13 \%. Dennoch wurden in vielen vergleichbaren Studien auch deutlich höhere Werte unpräparierter Oberfläche von 31-53 \% angegeben (De-Deus et al. 2015; De-Deus et al. 2019a; Peters et al. 2001a; Yin et al. 2010).

Peters et al. (2001a) beschrieben zudem, dass unterschiedliche Wurzelkanalanatomien und damit einhergehend unterschiedlich schwierige Anforderungen bei der Wurzelkanalpräparation einen größeren Einfluss auf den Anteil der unpräparierten Oberfläche bei einer Wurzelkanalpräparation haben, als die Technik der Präparation selbst. 


\subsubsection{Veränderung des Volumens}

Die präoperativen Volumina beider mesialen Wurzelkanäle der Versuchszähne lagen mittelwertig zwischen 3,28 $\pm 1,41 \mathrm{~mm}^{3}$ und 3,42 $\pm 2,41 \mathrm{~mm}^{3}$. Das volume of interest lag dabei $-1 \mathrm{~mm}$ vom Apex entfernt bis zur Schmelz-Zement-Grenze. Das Ausgangsvolumen war größer als in vergleichbaren Studien. Gagliardi et al. (2015), Poly et al. (2019) und Marceliano-Alves et al. (2015) beschrieben ein mittelwertiges Wurzelkanalvolumen von $1,71 \pm 0,86 \mathrm{~mm}^{3}$ bis $1,84 \pm 0,55 \mathrm{~mm}^{3}, 1,28$ $\pm 0,54 \mathrm{~mm}^{3}$ bis $1,76 \pm 0,53 \mathrm{~mm}^{3}$ und $1,68 \pm 1,48 \mathrm{~mm}^{3}$ bis $2,61 \pm 1,91 \mathrm{~mm}^{3} \mathrm{ge}-$ messen von der Furkation bis zum Apex.

Da anatomisch die Schmelz-Zement-Grenze weiter koronal als die Furkation lokalisiert ist, können die höheren Ausgangsvolumina in den anderen Studien erklärt werden. Zudem wurden alle akzessorischen Seitenkanäle im Ausgangsvolumen mit einbezogen. Vereinzelte Studien verzichteten auf den Einbezug der Seitenkanäle, was ebenfalls in geringeren Ausgangsvolumina resultiert (Siqueira et al. 2013).

Durch die Wurzelkanalpräparation wurde in allen Versuchszähnen das Wurzelkanalvolumen vergrößert. RECIPROC und WOG zeigten dabei nahezu identische Volumenzunahmen des Wurzelkanals von 197,4 $\pm 104,05 \%$ und 197,32 \pm $124,73 \%$. S1 Plus zeigte den größten Volumenzuwachs von 216,19 $\pm 165,37 \%$. Da keine vergleichbaren Studien mit S1 Plus vorliegen, muss auf Basis dieser Studie von einem geringgradig aggressiveren Schneidverhalten der Feile S1 Plus ausgegangen werden. Zur Untermauerung dieser These sind aber weitere Studien notwendig. Die Nullhypothese kann dennoch unterstützt werden, da keine signifikanten Unterschiede zwischen den Versuchsgruppen und den Krümmungen vorliegen.

Marceliano-Alves et al. (2015) beobachteten bei RECIPROC bei der Präparation mesialer Wurzeln von Unterkiefermolaren eine mittelwertige Volumenzunahme von $152,5 \pm 92,1 \%$. Stringheta et al. (2019) beobachteten einen prozentualen Volumenanstieg von lediglich $82.91 \pm 52,77 \%$ bei der Verwendung von RECIPROC. Die Volumenzunahme bei der Verwendung von WOG wurde mit 118,8 $\pm 59,91 \%$ angegeben. Es zeigten sich ebenfalls keine signifikanten Unterschiede zwischen den Instrumenten.

Alle genannten Werte liegen unter dem prozentualen Volumenzuwachs der vorliegenden Studie. 


\subsubsection{Veränderung des Structure Model Index}

Die Veränderung des SMI beurteilt die Wurzelkanalgeometrie, ohne auf physikalische Abmessungen Rücksicht zu nehmen (Hildebrand und Rüegsegger 1997a; Peters et al. 2000; Stringheta et al. 2019). Die präoperativen Werte des SMI waren in allen Versuchsgruppen mit den Werten 1,86 \pm 0,56 (S1 Plus), 1,88 \pm 0,34 (RECIPROC), 1,90 \pm 0,51 (WOG) ähnlich. Durch die Wurzelkanalpräparation werden höhere Werte des SMI erwartet, da die Wurzelkanalgeometrie postoperativ konvexere Flächen aufweisen soll und diese in einem höheren SMI resultieren (Hildebrand und Rüegsegger 1997a).

Die postoperativen Werte des SMI erhöhten sich um ein $\Delta$ SMI von 0,66 $\pm 0,26$ auf 2,52 $\pm 0,65$ für $\mathrm{S} 1$ Plus. Die Werte erhöhten sich um 0,65 $\pm 0,28$ auf 2,54 \pm 0,48 für RECIPROC und um 0,69 $\pm 0,26$ auf 2,59 $\pm 0,49$ für WOG. Zwischen den Gruppen gab es keine signifikanten Unterschiede. Die Werte entsprechen laut SMI einer runden, konischen Kegelform der Wurzelkanalgeometrie (MarcelianoAlves et al. 2015; Versiani et al. 2013).

Vergleichbare Studien erreichten bei der Präparation von Wurzelkanälen ähnliche postoperative Werte. Stringheta et al. (2019) gaben nach der Präparation mit RECIPROC und WOG einen SMI von 2,51 $\pm 0,73$ und 2,25 $\pm 0,51$ an. Auffallend waren die höheren Werte des SMI bei reziprok arbeitenden Präparationsfeilen. Rotierend arbeitende Präparationsfeilen hatten stets geringere Werte des SMI. Zudem erreichten bei Stringheta et al. (2019) ebenfalls die mit WOG präparierten Versuchszähne den höchsten postoperativen SMI. Die parallelogrammartige Querschnittsform der Präparationsfeile führt zu einer abwechselnden Berührung der Wurzelkanalwand mit einer oder zwei Schneidekanten und könnte somit größere Konizitäten provozieren (Stringheta et al. 2019; Versiani et al. 2018).

Andere Studien zeigten höhere postoperative Werte des SMI. Marceliano-Alves et al. (2015) beschrieben Werte von 2,91-3,05, Moore et al. (2009) gaben Werte von 2,63-2,83 an. Eine Erklärung kann in der komplexeren Wurzelkanalgeometrie der Versuchszähne mit doppelten Krümmungen liegen. Indes finden sich in Studien mit Verwendung von Oberkiefermolaren geringere Erhöhungen des SMI: You et al. (2011) ermittelten bei der Verwendung des rotierenden Feilensystems ProTaper (Dentsply Maillefer, Ballaigues, Schweiz) einen $\Delta$ SMI von 0,45 $\pm 1,64$. Paqué et al. (2009) ermittelten bei der Präparation mit mehreren rotierenden Feilensystemen einen $\Delta$ SMI von 0,02 $\pm 0,22$ (FlexMaster (VDW, München)) bis 0,46 \pm 0,38 (ProFile (Dentsply Maillefer, Ballaigues, Schweiz)). Auch Kim et al. (2013) erzielten bei der Präparation von Oberkiefermolaren geringere Werte des $\Delta \mathrm{SMI}$. Verwendet wurden WaveOne und ProTaper (Dentsply Maillefer, Ballaigues, 
Schweiz) in reziproker Arbeitsweise mit Werten von 0,53 $\pm 0,71$ und 0,42 $\pm 1,33$. Es wird deutlich, dass die Präparation von Oberkiefermolaren im Vergleich zu der vorliegenden Studie und anderen vergleichbaren Studien mit der Präparation von Unterkiefermolaren stets geringere Verbesserungen des SMI und somit weniger konvex präparierte Wurzelkanäle erzielen. Dies kann ebenfalls auf die komplexere Wurzelkanalanatomie zurückzuführen sein. Eine signifikant bessere Präparation wird bei der Verwendung reziprok arbeitender Instrumente gegenüber rotierend arbeitenden Feilen nicht erreicht (Kim et al. 2013; Marceliano-Alves et al. 2015; You et al. 2011).

\subsubsection{Veränderung von Canal Transportation und Centering Ability}

Die Messung der CT erfolgte an vier Punkten entlang des Wurzelkanals bei 1 $\mathrm{mm}, 3 \mathrm{~mm}, 5 \mathrm{~mm}$ und $7 \mathrm{~mm}$ vom Apex entfernt und separat für den mesiobukkalen und mesiolingualen Kanal. Viele vergleichbare Studien verwendeten nur drei oder weniger Messpunkte zur Bestimmung der CT und CA (da Silva Limoeiro et al. 2016; de Carvalho et al. 2015; Gergi et al. 2014; Pinheiro et al. 2018; Poly et al. 2019).

Durch mehrere Messpunkte an den Wurzelkanalquerschnitten sollten genauere Werte erzielt werden, da unter anderem durch die Verwendung von Versuchszähnen mit doppelten Wurzelkanalkrümmungen in beiden Teilen der Wurzel gegenläufige Wurzelkanalbegradigungen erwartet wurden.

Die Wurzelkanalfeile besitzt bei einer Legierung aus NiTi die metallurgische Eigenschaft, sich nach Deformation wieder in die Ausgangsposition zurückzustellen (memory shape) (Thompson 2000). Daraus kann die Tendenz einer nach mesial verschobenen Wurzelkanalbegradigung bei der Präparation vermutet werden.

Erkennbar ist die von apikal nach koronal zunehmende negative CT in allen Feilensystemen der Studie, was einer Distalverlagerung des ursprünglichen Wurzelkanalverlaufs entspricht. In allen Gruppen wurde eine signifikante Erhöhung der CT von apikal nach koronal beobachtet. Signifikante Unterschiede zwischen den Gruppen mit einfachen und doppelten Wurzelkanalkrümmungen wurden nicht festgestellt. Die höchste mittelwertige CT wurde für RECIPROC mit 0,08 mm ermittelt. Die beiden anderen Feilengruppen folgten mit einer mittelwertigen Verlagerung von $0,07 \mathrm{~mm}$.

Marceliano-Alves et al. (2015) maßen mit RECIPROC ebenfalls die größte CT mit 0,11 $\pm 0,08 \mathrm{~mm}$ im Vergleich zu den anderen verwendeten Feilensystemen. 
Es traten signifikant höhere Werte der CT bei der Verwendung reziprok arbeitender Feilen gegenüber rotierend arbeitenden Feilen auf. Als mögliche Erklärung wird die Hitzebearbeitung der Metalllegierung von Feilensystemen angesehen (Pongione et al. 2012; Saber et al. 2015).

In der vorliegenden Studie verwendete WOG als einziges System die hitzebearbeitete Gold-Wire-Legierung. Signifikant bessere Ergebnisse wurden mit diesem Feilensystem jedoch nicht beobachtet.

Poly et al. (2019) ermittelten eine signifikant bessere CT bei der Querschnittsmessung bei $7 \mathrm{~mm}$ koronal des Apex für das Feilensystem XP-endo Shaper (XPS; FKG, La Chaux-de-Fonds, Schweiz) gegenüber WOG. Eine Überlegenheit der Gold-Wire-Legierung wurde auch hier nicht festgestellt. Poly et al. (2019) gaben zudem deutlich höhere Werte von $0,11 \pm 0,12 \mathrm{~mm}$ bis $0,19 \pm 0,07 \mathrm{~mm}$ für die CT an.

Höhere Werte der CT wurden ebenfalls in mehreren vergleichbaren Studien beobachtet (de Almeida et al. 2015; Gagliardi et al. 2015; Pedullà et al. 2016; Silva et al. 2017).

In keiner vergleichbaren Studie wurde eine von apikal nach koronal zunehmende distalwärts gerichtete CT beobachtet. Bei standardisiertem Präparationsprotokoll und standardisierter Messtechnik kann eine leicht nach distal geneigte Feilenhaltung während der Präparation eine Erklärung für die Werte sein. Hofmann (2019) unterstützt die These, da sich in seiner Studie der Einfluss des Operateurs auf die CT größer herausstellte, als der Einfluss der Feilensysteme selbst.

Das Präparationsverhalten im apikalen Bereich der Wurzelkanäle zeigt eine sehr geringe mittelwertige CT von 0,01 $\pm 0,08 \mathrm{~mm}$ (S1 Plus) bis 0,03 $\pm 0,06 \mathrm{~mm}$ (RECIPROC). Eine geringe CT bei der klinischen Wurzelkanalaufbereitung ist relevant, da eine CT über $0,3 \mathrm{~mm}$ einen negativen Einfluss auf die Dichtigkeit von Wurzelfüllmaterialien hat (Wu et al. 2000).

Die CA zeigte im Gegensatz zur CT keine signifikanten Unterschiede zwischen den Gruppen. Auch wurde von apikal nach koronal keine schlechtere Zentrierung festgestellt.

Die besten mittelwertigen Ergebnisse für die CA erzielte WOG mit 0,65 $\pm 0,24$ vor der RECIPROC mit 0,63 $\pm 0,23$ und S1 Plus mit 0,60 $\pm 0,25$.

Im Vergleich mit anderen Studien kann eine Verbesserung der CA von koronal nach apikal beobachtet werden. Pedullà et al. (2016) berechneten bei der Verwendung von Mtwo eine von koronal nach apikal verlaufende Verbesserung von $0,33 \pm 0,14$ auf 0,68 $\pm 0,09$. Bei der Verwendung des Feilensystems Twisted File 
Adaptive wurde eine Verbesserung von 0,32 $\pm 0,04$ auf 0,59 $\pm 0,14$ berechnet. Poly et al. (2019) zeigten die gleiche Veränderung von koronal nach apikal bei der Präparation mit WOG mit Werten von 0,33 $\pm 0,13$ bis 0,56 $\pm 0,32$. Der XPendo Shaper erzielte Werte von koronal nach apikal von $0,52 \pm 0,21$ bis $0,64 \pm$ 0,21 .

Mehrere Studien zeigten niedrigere Werte für die CA, was unter anderem an einer unterschiedlichen Präparationstechnik durch unterschiedliche Anwender liegen kann (da Silva Limoeiro et al. 2016; de Carvalho et al. 2015; Pedullà et al. 2016; Silva et al. 2017). 


\section{$5 \quad$ Zusammenfassung}

Das wesentliche Ziel einer mechanischen Wurzelkanalpräparation ist neben der möglichst vollständigen Entfernung des bakteriell infizierten Dentins die Erhaltung der ursprünglichen Wurzelkanalanatomie. Auf eine ausreichende Instrumentierung des Wurzelkanals sollte zudem geachtet werden. Zu kleine Präparationsgrößen verursachen einen zu großen Anteil an unpräparierter Oberfläche des Wurzelkanals. Eine Überinstrumentierung kann andererseits zu einer Schwächung der Zahnhartsubstanz führen.

Unter Berücksichtigung der genannten Aspekte untersuchte die vorliegende Studie das Präparationsverhalten dreier Einfeilensysteme aus NiTi-Legierungen mit reziproker Arbeitsweise in einfach und doppelt gekrümmten mesialen Wurzelkanälen von ersten Unterkiefermolaren.

75 extrahierte Versuchszähne mit Wurzelkanalkrümmungen von $15-40^{\circ}$ und Krümmungsradien bis einschließlich $18 \mathrm{~mm}$ wurden in matched triples in drei homogene Gruppen ( $n=25$ ) aufgeteilt. Als Untergruppierung wurden pro Gruppe jeweils elf Versuchszähne mit doppelten Wurzelkanalkrümmungen und 14 mit einer einzelnen Krümmung verwendet. Die Präparation der Wurzelkanäle erfolgte durch die Feilensysteme WOG, RECIPROC und S1 Plus in einem standardisierten Präparations- und Spülprotokoll.

Prä- und postoperativ erfolgten Scans mit dem Mikro-CT Bruker SkyScan 1272. Dabei wurden folgende Parameter berechnet: Veränderung der Wurzelkanaloberfläche mit Berechnung der unpräparierten Oberfläche, Veränderung des Wurzelkanalvolumens, des Structure Model Index, Canal Transportation und der Centering Ability.

In der Nullhypothese wurde erwartet, dass keine Unterschiede im Präparationsergebnis für die drei Feilensysteme vorliegen.

Die Veränderungen von Volumen, Oberfläche, SMI und Centering Ability zeigten keine signifikanten Unterschiede zwischen den Gruppen ( $p>0,05)$. Es zeigten sich keine signifikanten Unterschiede zwischen der Präparation von Versuchszähnen mit einfachen und doppelten Wurzelkanalkrümmungen.

Bei der Canal Transportation wurden keine signifikanten Veränderungen zwischen den Feilengruppen festgestellt. Es zeigten sich jedoch in allen Gruppen signifikante Veränderungen der Canal Transportation von apikal nach koronal. Es wurden keine Instrumentenfrakturen während der Präparation festgestellt. Die Studie zeigte insgesamt eine gute Präparation durch alle Feilensysteme. Die aufgestellte Nullhypothese konnte dabei vollständig bestätigt werden. 


\section{$6 \quad$ Literaturverzeichnis}

Al-Fouzan KS (2003): Incidence of rotary ProFile instrument fracture and the potential for bypassing in vivo. Int Endod J $\underline{36}, 864-867$

Al-Obaida MI, Merdad K, Alanazi MS, Altwaijry H, AlFaraj M, Alkhamis AA, AlMadi EM (2019): Comparison of cyclic fatigue resistance of 5 heat-treated nickeltitanium reciprocating systems in canals with single and double curvatures. $\mathrm{J}$ Endod $\underline{45}, 1237-1241$

Al-Omari MAO, Dummer PMH (1995): Canal blockage and debris extrusion with eight preparation techniques. J Endod 21, 154-158

Al-Sudani D, Grande NM, Plotino G, Pompa G, Di Carlo S, Testarelli L, Gambarini G (2012): Cyclic fatigue of nickel-titanium rotary instruments in a double (sshaped) simulated curvature. J Endod $\underline{38}$, 987-989

Alapati SB, Brantley WA, lijima M, Clark WAT, Kovarik L, Buie C, Liu J, Johnson WB (2009): Metallurgical characterization of a new nickel-titanium wire for rotary endodontic instruments. J Endod 35, 1589-1593

Alovisi M, Cemenasco A, Mancini L, Paolino D, Scotti N, Bianchi CC, Pasqualini D (2017): Micro-CT evaluation of several glide path techniques and ProTaper Next shaping outcomes in maxillary first molar curved canals. Int Endod J $\underline{50}$, 387-397

Alovisi M, Pasqualini D, Musso E, Bobbio E, Giuliano C, Mancino D, Scotti N, Berutti E (2018): Influence of contracted endodontic access on root canal geometry: An in vitro study. J Endod 44, 614-620

Amoroso-Silva P, Alcalde MP, Hungaro Duarte MA, De-Deus G, Ordinola-Zapata R, Freire LG, Cavenago BC, De Moraes IG (2017): Effect of finishing instrumentation using $\mathrm{NiTi}$ hand files on volume, surface area and uninstrumented surfaces in c-shaped root canal systems. Int Endod J $\underline{50}$, 604611

Aydin ZU, Keskin NB, Özyürek T, Geneci F, Ocak M, Çelik HH (2019): Microcomputed assessment of transportation, centering ratio, canal area, and volume increase after single-file rotary and reciprocating glide path instrumentation in curved root canals: a laboratory study. J Endod $\underline{45}, 791-796$ 
Bach M, Hülsmann M (2019): Single-File-Systeme - Eine vergleichende Übersicht. Endodontie 28, 161-173

Backman CA, Oswald RJ, Pitts DL (1992): A radiographic comparison of two root canal instrumentation techniques. J Endod $\underline{18}, 19-24$

Barrett J, Keat N (2004): Artifacts in CT: recognition and avoidance. Radiographics $\underline{24}, 1679-1691$

Belladonna FG, Carvalho MS, Cavalcante DM, Fernandes JT, de Carvalho Maciel AC, Oliveira HE, Lopes RT, Silva EJNL, De-Deus G (2018): Microcomputed tomography shaping ability assessment of the new blue thermal treated reciproc instrument. J Endod $\underline{44}$, 1146-1150

Bergmans L, van Cleynenbreugel J, Wevers M, Lambrechts P (2001): A methodology for quantitative evaluation of root canal instrumentation using microcomputed tomography. Int Endod J 34, 390-398

Bonaccorso A, Cantatore G, Condorelli GG, Schäfer E, Tripi TR (2009): Shaping ability of four nickel-titanium rotary instruments in simulated s-shaped canals. J Endod 35, 883-886

Bramante CM, Berbert A, Borges RP (1987): A methodology for evaluation of root canal instrumentation. J Endod 13, 243-245

Bruker Corporation (2016): NRecon user manual http://brukermicroct.com/next/NReconUserGuide.pdf; abgerufen am 13.02.2018

Bruker Corporation (2018): Skyscan 1272 high-resolution micro-CT https://www.bruker.com/products/microtomography/micro-ct-for-samplescanning/skyscan-1272/overview.html; abgerufen am 11.2.2018

Bürklein S, Donnermeyer D, Schäfer E (2017): Nickel-Titan-Instrumente rotierend, vibrierend, reziprokierend. Endodontie 26, 299-307

Bürklein S, Flüch S, Schäfer E (2019): Shaping ability of reciprocating single-file systems in severely curved canals: WaveOne and Reciproc versus WaveOne Gold and Reciproc blue. Odontology 107, 96-102 
Bürklein S, Hinschitza K, Dammaschke T, Schäfer E (2012): Shaping ability and cleaning effectiveness of two single-file systems in severely curved root canals of extracted teeth: Reciproc and WaveOne versus Mtwo and ProTaper. Int Endod J 노, 449-461

Bürklein S, Schäfer E (2013): Critical evaluation of root canal transportation by instrumentation. Endod Topics 29, 110-124

Burroughs JR, Bergeron BE, Roberts MD, Hagan JL, Himel VT (2012): Shaping ability of three nickel-titanium endodontic file systems in simulated s-shaped root canals. J Endod $\underline{38}$, 1618-1621

Calhoun G, Montgomery S (1988): The effects of four instrumentation techniques on root canal shape. J Endod 14, 273-277

Cassimiro M, Romeiro K, Gominho L, de Almeida A, Silva L, Albuquerque D (2018): Effects of Reciproc, ProTaper Next and WaveOne Gold on root canal walls: a stereomicroscope analysis. Iran Endod J $\underline{13}$, 228-233

Cheung GS (2009): Instrument fracture: mechanisms, removal of fragments, and clinical outcomes. Endod Topics 16, 1-26

Clem WH (1969): Endodontics: the adolescent patient. Dent Clin North Am $\underline{13}$, 482-493

Crump MC, Natkin E (1970): Relationship of broken root canal instruments to endodontic case prognosis: a clinical investigation. J Am Dent Assoc 80, 13411347

Cunningham CJ, Senia ES (1992): A three-dimensional study of canal curvatures in the mesial roots of mandibular molars. J Endod 18, 294-300

da Silva Limoeiro AG, dos Santos AHB, De Martin AS, Kato AS, Fontana CE, Gavini G, Freire LG, Bueno CEDS (2016): Micro-computed tomographic evaluation of 2 nickel-titanium instrument systems in shaping root canals. $\mathrm{J}$ Endod 42, 496-499

de Albuquerque MS, Nascimento AS, Gialain IO, de Lima EA, Nery JA, de Souza Araujo PR, de Menezes RF, Kato AS, Braz R (2019): Canal transportation, centering ability, and dentin removal after instrumentation: a micro-CT evaluation. J Contemp Dent Pract 20, 806-811 
de Almeida BC, Ormiga F, de Araújo MCP, Lopes RT, Lima ICB, dos Santos BC, Gusman H (2015): Influence of heat treatment of nickel-titanium rotary endodontic instruments on apical preparation: a micro-computed tomographic study. J Endod 41, 2031-2035

de Carvalho GM, Sponchiado EC, Jr., Bittencourt Garrido AD, Lia RCC, Garcia LFR, Marques AAF (2015): Apical transportation, centering ability, and cleaning effectiveness of reciprocating single-file system associated with different glide path techniques. J Endod 41, 2045-2049

de Pablo OV, Estevez R, Péix Sánchez M, Heilborn C, Cohenca N (2010): Root anatomy and canal configuration of the permanent mandibular first molar: a systematic review. J Endod $\underline{36}$, 1919-1931

De-Deus G, Belladonna FG, Silva EJNL, Marins JR, Souza EM, Perez R, Lopes RT, Versiani MA, Paciornik S, de Almeida Neves A (2015): Micro-CT evaluation of non-instrumented canal areas with different enlargements performed by NiTi systems. Braz Dent J 트, 624-629

De-Deus G, Belladonna FG, Silva EJNL, Vieira VTL, Elias CN, Plotino G, Grande NM (2017): Blue thermomechanical treatment optimizes fatigue resistance and flexibility of the reciproc files. J Endod $\underline{43}, 462-466$

De-Deus G, Belladonna FG, Simões-Carvalho M, Cavalcante DM, Ramalho CNMJ, Souza EM, Lopes RT, Silva EJNL (2019a): Shaping efficiency as a function of time of a new heat-treated instrument. Int Endod J $\underline{52}$, 337-342

De-Deus G, Belladonna FG, Zuolo AS, Cavalcante DM, Simões-Carvalho M, Marinho A, Souza EM, Lopes RT, Silva EJNL (2019b): 3-dimensional ability assessment in removing root filling material from pair-matched oval-shaped canals using thermal-treated instruments. J Endod $\underline{45}, 1135-1141$

De-Deus G, Simões-Carvalho M, Belladonna FG, Cavalcante DM, Portugal LS, Prado CG, Souza EM, Lopes RT, Silva EJNL (2020): Arrowhead design ultrasonic tip as a supplementary tool for canal debridement. Int Endod J $\underline{53}, 410-$ 420

Delai D, Jardine AP, Mestieri LB, Boijink D, Fontanella VRC, Grecca FS, Kopper PMP (2019): Efficacy of a thermally treated single file compared with rotary systems in endodontic retreatment of curved canals: a micro-CT study. Clin Oral Investig 23, 1837-1844 
Dentsply Sirona (2018): Gebrauchsanweisung WaveOne Gold System http://www.dentsplymaillefer.com/wp-

content/uploads/2017/03/Dentsply_Maillefer_WAVEONE_GOLD_SYSTEM_DF U_0117_WEB_DSE_DE.pdf; abgerufen am 16.04.2018

DGZMK (2000): Stellungnahme der DGZMK und der DGZ: Wurzelkanalaufbereitung. Dtsch Zahnärztl Z $\underline{55}$

Dittmer P: Die maschinelle Aufbereitung des Wurzelkanals mit dem Tri Auto ZX Winkelstück und FlexMaster-Instrumenten. Med. Diss. Greifswald 2003

Dowker SEP, Davis GR, Elliott JC (1997): X-ray microtomography: nondestructive three-dimensional imaging for in vitro endodontic studies. Oral Surg Oral Med Oral Pathol Oral Radiol Endod 3ㅜ 510-516

Drukteinis S, Peciuliene V, Dummer PMH, Hupp J (2019): Shaping ability of BioRace, ProTaper NEXT and Genius nickel-titanium instruments in curved canals of mandibular molars: a micro-CT study. Int Endod J $\underline{52}, 86-93$

Duke F, Shen Y, Zhou H, Ruse ND, Wang ZJ, Hieawy A, Haapasalo M (2015): Cyclic fatigue of ProFile Vortex and Vortex Blue nickel-titanium files in single and double curvatures. J Endod 노, 1686-1690

Duque JA, Vivan RR, Duarte MAH, Alcalde MP, Cruz VM, Borges MMB, Bramante CM (2019): Effect of larger apical size on the quality of preparation in curved canals using reciprocating instruments with different heat thermal treatments. Int Endod J 모, 1652-1659

Eggert M, Gernhardt C (2012): Entfernung frakturierter Instrumente - Eine Literaturübersicht. Endodontie 21, 351-359

Elashiry MM, Saber SE, Elashry SH (2020): Comparison of shaping ability of different single-file systems using microcomputed tomography. Eur J Dent $\underline{14}$, 70-76

Elliott JC, Dover SD (1982): X-ray microtomography. J Microsc 126, 211-213

Elnaghy AM, Elsaka SE (2016): Mechanical properties of ProTaper Gold nickeltitanium rotary instruments. Int Endod J $\underline{49}, 1073-1078$ 
Elnaghy AM, Elsaka SE (2018): Cyclic fatigue resistance of One Curve, 2Shape, ProFile Vortex, Vortex Blue, and RaCe nickel-titanium rotary instruments in single and double curvature canals. J Endod $\underline{44}, 1725-1730$

Elsaka SE, Elnaghy AM, Badr AE (2017): Torsional and bending resistance of WaveOne Gold, Reciproc and Twisted File Adaptive instruments. Int Endod J $\underline{50}$, 1077-1083

Erlandsson K, Buvat I, Pretorius PH, Thomas BA, Hutton BF (2012): A review of partial volume correction techniques for emission tomography and their applications in neurology, cardiology and oncology. Phys Med Biol $\underline{57}$, R119-159

Espir CG, Nascimento-Mendes CA, Guerreiro-Tanomaru JM, Freire LG, Gavini G, Tanomaru-Filho M (2017): Counterclockwise or clockwise reciprocating motion for oval root canal preparation: a micro-CT analysis. Int Endod J $\underline{51}, 541$ 548

European Society of Endodontology (2006): Quality guidelines for endodontic treatment: consensus report of the European Society of Endodontology. Int Endod J $\underline{39}, 921-930$

Eyuboglu TF, Olcay K, Özcan M (2017): A clinical study on single-visit root canal retreatments on consecutive 173 patients: frequency of periapical complications and clinical success rate. Clin Oral Investig 21, 1761-1768

Farzaneh M, Abitbol S, Lawrence HP, Friedman S (2004): Treatment outcome in endodontics - the Toronto Study. Phase II: initial treatment. J Endod 30, 302-309

Feldkamp LA, Davis LC, Kress JW (1984): Practical cone-beam algorithm. J Opt Soc Am A Opt Image Sci Vis 1, 612-619

Ferraz CC, Gomes NV, Gomes BP, Zaia AA, Teixeira FB, Souza-Filho FJ (2001): Apical extrusion of debris and irrigants using two hand and three engine-driven instrumentation techniques. Int Endod J 34, 354-358

Fox A, Basrani B, Kishen A, Lam EWN (2018): A novel method for characterizing beam hardening artifacts in cone-beam computed tomographic images. J Endod $\underline{44}, 869-874$

Gagliardi J, Versiani MA, de Sousa-Neto MD, Plazas-Garzon A, Basrani B (2015): Evaluation of the shaping characteristics of ProTaper Gold, ProTaper NEXT, and ProTaper Universal in curved canals. J Endod 41, 1718-1724 
Gambill JM, Alder M, del Rio CE (1996): Comparison of nickel-titanium and stainless steel hand-file instrumentation using computed tomography. J Endod 22, 369-375

Gao Y, Gutmann JL, Wilkinson K, Maxwell R, Ammon D (2012): Evaluation of the impact of raw materials on the fatigue and mechanical properties of ProFile Vortex rotary instruments. J Endod $\underline{38}, 398-401$

Gao Y, Shotton V, Wilkinson K, Phillips G, Johnson WB (2010): Effects of raw material and rotational speed on the cyclic fatigue of ProFile Vortex rotary instruments. J Endod $\underline{36}, 1205-1209$

Gergi R, Arbab-Chirani R, Osta N, Naaman A (2014): Micro-computed tomographic evaluation of canal transportation instrumented by different kinematics rotary nickel-titanium instruments. J Endod 40, 1223-1227

Giansiracusa Rubini A, Plotino G, Al-Sudani D, Grande NM, Sonnino G, Putorti E, Cotti E, Testarelli L, Gambarini G (2014): A new device to test cutting efficiancy of mechanical endodontic instruments. Med Sci Monit 20, 374-378

Glickmann GN, Dumsha TC: Problems in canal cleaning and shaping; In: Gutmann JL, Dumsha TC, Lovdahl PE, Hovland EJ (Hrsg.): Problem solving in endodontics. 3rd edn. St Louis, MO: Mosby 1997, 91-122

Gluskin AH, Brown DC, Buchanan LS (2001): A reconstructed computerized tomographic comparison of Ni-Ti rotary GT files versus traditional instruments in canals shaped by novice operators. Int Endod J $\underline{34}$, 476-484

Goerig AC, Michelich RJ, Schultz HH (1982): Instrumentation of root canals in molar using the step-down technique. J Endod $\underline{8}, 550-554$

Greene KJ, Krell KV (1990): Clinical factors associated with ledged canals in maxillary and mandibular molars. Oral Surg Oral Med Oral Pathol $\underline{70}$, 490-497

Gu Y, Lu Q, Wang P, Ni L (2010): Root canal morphology of permanent threerooted mandibular first molars: Part II - measurement of root canal curvatures. J Endod $\underline{36}, 1341-1346$

Gündogar M, Özyürek T (2017): Cyclic fatigue resistance of OneShape, HyFlex EDM, WaveOne Gold, and Reciproc Blue nickel-titanium instruments. J Endod $\underline{43}, 1192-1196$ 
Haapasalo M, Shen Y (2013): Evolution of nickel-titanium instruments: from past to future. Endod Topics $\underline{29}$, 3-17

Heuer MA (1963): The biomechanics of endodontic therapy. Dent Clin North Am 7, 341-359

Hieawy A, Haapasalo M, Zhou H, Wang ZJ, Shen Y (2015): Phase transformation behavior and resistance to bending and cyclic fatigue of ProTaper Gold and ProTaper Universal instruments. J Endod 411, 1134-1138

Hildebrand T, Rüegsegger P (1997a): Quantification of bone microarchitecture with the structure model index. Comput Methods Biomech Biomed Engin 1, 1523

Hildebrand T, Rüegsegger P (1997b): A new method for the model-independent assessment of thickness in three-dimensional images. J Microsc $\underline{185}, 67-75$

Hiller S: Vergleichende In-vitro-Studie zur maschinellen Wurzelkanalpräparation mit den NiTi-Single-File-Systemen RECIPROC, Wave One und One Shape. Med. Diss. Göttingen 2014

Hofmann N (2019): Both operator and heat treatment determine the centring ability of Reciproc (R) files in vitro. Clin Oral Investig 23, 697-706

Hu W, Whitten B, Sedgley C, Svec T (2014): Effect of three NiTi files on transportation of the apical foramen. Int Endod J $\underline{47}, 1064-1071$

Hübscher W, Barbakow F, Peters OA (2003): Root-canal preparation with FlexMaster: canal shapes analysed by micro-computed tomography. Int Endod J $\underline{36}, 740-747$

Hülsmann M: Entwicklung einer Methode zur standardisierten Überprüfung verschiedener Aufbereitungsparameter und vergleichende In-vitro-Untersuchung unterschiedlicher Systeme zur maschinellen Wurzelkanalaufbereitung. Med. Habil.-Schr. Göttingen 1998

Hülsmann M (2006): Die Desinfektion des endodontischen Systems. Endodontie 15, 147-168 
Hülsmann M: Wurzelkanalpräparation; In: Hülsmann M (Hrsg.): Checklisten der Zahnmedizin - Endodontie. Thieme Verlag, Stuttgart 2008, 129-139

Hülsmann M (2013): Effects of mechanical instrumentation and chemical irrigation on the root canal dentin and surrounding tissues. Endod Topics $\underline{29}, 55-$ 86

Hülsmann M, Herbst U, Schäfers F (2003): Comparative study of root-canal preparation using Lightspeed and Quantec SC rotary NiTi instruments. Int Endod J $\underline{36}, 748-756$

Hülsmann M, Peters OA, Dummer PMH (2005): Mechanical preparation of root canals: shaping goals, techniques and means. Endod Topics $\underline{10}, 30-76$

Hülsmann M, Schinkel I (1999): Influence of several factors on the success or failure of removal of fractured instruments from the root canal. Endod Dent Traumatol $\underline{15}, 252-258$

Ingle JI (1961): A standardized endodontic technique utilizing newly designed instruments and filling materials. Oral Surg Oral Med Oral Pathol $\underline{14}$, 83-91

Iqbal MK, Kohli MR, Kim JS (2006): A retrospective clinical study of incidence of root canal instrument separation in an endodontics graduate program: a PennEndo database study. J Endod 32, 1048-1052

Kapalas A, Lambrianidis T (2000): Factors associated with root canal ledging during instrumentation. Endod Dent Traumatol 16, 229-231

Kazemi RB, Stenman E, Spångberg LS (1996): Machining efficiency and wear resistance of nickel-titanium endodontic files. Oral Surg Oral Med Oral Pathol Oral Radiol Endod 81, 596-602

Keskin C, Inan U, Demiral M, Keles A (2017): Cyclic fatigue resistance of Reciproc Blue, Reciproc, and WaveOne Gold Reciprocating instruments. $\mathrm{J}$ Endod $\underline{43}, 1360-1363$

Keskin C, Sivas Yilmaz Ö, Inan U, Özdemir Ö (2019): Postoperative pain after glide path preparation using manual, reciprocating and continuous rotary instruments: a randomized clinical trial. Int Endod J $\underline{52}, 579-587$ 
Kim HC, Hwang YJ, Jung DW, You SY, Kim HC, Lee W (2013): Micro-computed tomography and scanning electron microscopy comparisons of two nickeltitanium rotary root canal instruments used with reciprocating motion. Scanning $\underline{35}, 112-118$

Linßen F: Eine vergleichende In-vitro-Studie zur maschinellen Wurzelkanalpräparation mit den Nickel-Titan-Systemen S5, Mtwo und ProTaper Universal. Med. Diss. Göttingen 2013

Marceliano-Alves MFV, Sousa-Neto MD, Fidel SR, Steier L, Robinson JP, Pécora JD, Versiani MA (2015): Shaping ability of single-file reciprocating and heattreated multifile rotary systems: a micro-CT study. Int Endod J $\underline{48}, 1129-1136$

Mendonça de Moura JD, Bueno CEDS, Fontana CE, Pelegrine RA (2019): Extrusion of debris from curved root canals instrumented up to different working lengths using different reciprocating systems. J Endod $\underline{45}, 930-934$

Metzger Z, Zary R, Cohen R, Teperovich E, Paqué F (2010): The quality of root canal preparation and root canal obturation in canals treated with rotary versus self-adjusting files: a three-dimensional micro-computed tomographic study. J Endod 36, 1569-1573

Meyer G (1991): Die Aufbereitung des Wurzelkanals. Zahnärztl Mitt $\underline{81}$, 864-870

Miserendino LJ, Miserendino CA, Moser JB, Heuer MA, Osetek EM (1988): Cutting efficiency of endodontic instruments. Part III. Comparison of sonic and ultrasonic instrument systems. J Endod 14, 24-30

Miyazaki S, Otsuka K (1986): Deformation and transition behaviour associated with the R Phase in Ti-Ni alloys. Metall Trans A 17A, 53-63

Moore J, Fitz-Walter P, Parashos P (2009): A micro-computed tomographic evaluation of apical root canal preparation using three instrumentation techniques. Int Endod J 느, 1057-1064

Neelakantan P, Reddy P, Gutmann JL (2016): Cyclic fatigue of two different single files with varying kinematics in a simulated double-curved canal. J Investig Clin Dent $\underline{7}, 272-277$

O'Sullivan JDB, Behnsen J, Starborg T, MacDonald AS, Phythian-Adams AT, Else KJ, Cruickshank SM, Withers PJ (2018): X-ray micro-computed tomography $(\mu \mathrm{CT})$ : an emerging opportunity in parasite imaging. Parasitology $\underline{145}, 848-854$ 
Ørstavik D, Kerekes K, Molven O (1991): Effects of extensive apical reaming and calcium hydroxide dressing on bacterial infection during treatment of apical periodontitis: a pilot study. Int Endod J 24, 1-7

Özyürek T (2016): Cyclic fatigue resistance of Reciproc, WaveOne, and WaveOne Gold nickel-titanium instruments. J Endod 42, 1536-1539

Paqué F, Balmer M, Attin T, Peters OA (2010): Preparation of oval-shaped root canals in mandibular molars using nickel-titanium rotary instruments: a microcomputed tomography study. J Endod $\underline{36}, 703-707$

Paqué F, Ganahl D, Peters OA (2009): Effects of root canal preparation on apical geometry assessed by micro-computed tomography. J Endod $\underline{35}, 1056-1059$

Paqué F, Musch U, Hülsmann M (2005): Comparison of root canal preparation using $\mathrm{RaCe}$ and ProTaper rotary Ni-Ti instruments. Int Endod J $\underline{38}, 8-16$

Parashos P, Messer $\mathrm{HH}$ (2006): Rotary NiTi instrument fracture and its consequences. J Endod $\underline{32}, 1031-1043$

Pasqualini D, Alovisi M, Cemenasco A, Mancini L, Paolino DS, Bianchi CC, Roggia A, Scotti N, Berutti E (2015): Micro-computed tomography evaluation of ProTaper Next and BioRace shaping outcomes in maxillary first molar curved canals. J Endod $\underline{41}$, 1706-1710

Pedullà E, Grande NM, Plotino G, Gambarini G, Rapisarda E (2013): Influence of continuous or reciprocating motion on cyclic fatigue resistance of 4 different nickel-titanium rotary instruments. J Endod 39, 258-261

Pedullà E, Plotino G, Grande NM, Avarotti G, Gambarini G, Rapisarda E, Mannocci F (2016): Shaping ability of two nickel-titanium instruments activated by continuous rotation or adaptive motion: a micro-computed tomography study. Clin Oral Investig 20, 2227-2233

Pereira ESJ, Peixoto IFC, Viana ACD, Oliveira II, Gonzalez BM, Buono VTL, Bahia MGA (2012): Physical and mechanical properties of a thermomechanically treated $\mathrm{NiTi}$ wire used in the manufacture of rotary endodontic instruments. Int Endod J 노, 469-474 
Pereira ESJ, Viana ACD, Buono VTL, Peters OA, Bahia MGA (2015): Behavior of nickel-titanium instruments manufactured with different thermal treatments. J Endod $\underline{41}, 67-71$

Perez Morales MLN, González Sánchez JA, Olivieri Fernández JG, Laperre K, Abella Sans F, Jaramillo DE, Terol FD (2020): TRUShape versus XP-endo Shaper: a micro-computed tomographic assessment and comparative study of the shaping ability-an in vitro study. J Endod $\underline{46}, 271-276$

Peters OA, Boessler C, Paqué F (2010): Root canal preparation with a novel nickel-titanium instrument evaluated with micro-computed tomography: canal surface preparation over time. J Endod $\underline{36}, 1068-1072$

Peters OA, Laib A, Gohring TN, Barbakow F (2001a): Changes in root canal geometry after preparation assessed by high-resolution computed tomography. J Endod 27, 1-6

Peters OA, Laib A, Rüegsegger P, Barbakow F (2000): Three-dimensional analysis of root canal geometry by high-resolution computed tomography. J Dent Res $\underline{79}, 1405-1409$

Peters OA, Paqué F (2010): Current developments in rotary root canal instrument technology and clinical use: a review. Quintessence Int $\underline{41}$, 479-488

Peters OA, Peters Cl, Schönenberger K, Barbakow F (2003): ProTaper rotary root canal preparation: effects of canal anatomy on final shape analysed by micro CT. Int Endod J $\underline{36}, 86-92$

Peters OA, Schönenberger K, Laib A (2001b): Effects of four Ni-Ti preparation techniques on root canal geometry assessed by micro computed tomography. Int Endod J 34, 221-230

Pettiette MT, Metzger Z, Phillips C, Trope M (1999): Endodontic complications of root canal therapy performed by dental students with stainless-steel K-files and nickel-titanium hand files. J Endod 25, 230-234

Pineda F, Kuttler Y (1972): Mesiodistal and buccolingual roentgenographic investigation of 7,275 root canals. Oral Surg Oral Med Oral Pathol $\underline{33}, 101-110$

Pinheiro SR, Alcalde MP, Vivacqua-Gomes N, Bramante CM, Vivan RR, Duarte $\mathrm{MAH}$, Vasconcelos BC (2018): Evaluation of apical transportation and centring ability of five thermally treated NiTi rotary systems. Int Endod J $\underline{51}, 705-713$ 
Poly A, AlMalki F, Marques F, Karabucak B (2019): Canal transportation and centering ratio after preparation in severely curved canals: analysis by microcomputed tomography and double-digital radiography. Clin Oral Investig $\underline{23}$, 4255-4262

Pongione G, Pompa G, Milana V, Di Carlo S, Giansiracusa A, Nicolini E, De Angelis $F$ (2012): Flexibility and resistance to cyclic fatigue of endodontic instruments made with different nickel-titanium alloys: a comparative test. Ann Stomatol (Roma) $\underline{3}, 119-122$

Ramirez-Salomon M, Soler-Bientz R, de la Garza-Gonzalez R, Palacios-Garza CM (1997): Incidence of Lightspeed separation and the potential for bypassing. J Endod 23, 586-587

Roane JB, Sabala CL, Duncanson MG, Jr. (1985): The "balanced force" concept for instrumentation of curved canals. J Endod 11, 203-211

Robinson JP, Lumley PJ, Cooper PR, Grover LM, Walmsley AD (2013): Reciprocating root canal technique induces greater debris accumulation than a continuous rotary technique as assessed by 3-dimensional micro-computed tomography. J Endod 39, 1067-1070

Rödig T: Die Wurzelkanalpräparation; In: Hülsmann M, Nordmeyer S, Drebenstedt S (Hrsg.): Grundlagen der modernen Endodontie. Spitta Verlag, Balingen 2009, 123-174

Rödig T, Hausdörfer T, Konietschke F, Dullin C, Hahn W, Hülsmann M (2012): Efficacy of D-RaCe and ProTaper Universal Retreatment NiTi instruments and hand files in removing gutta-percha from curved root canals - a micro-computed tomography study. Int Endod J $\underline{45}, 580-589$

Rödig T, Wagner J, Wiegand A, Rizk M (2018): Efficacy of the ProTaper retreatment system in removing Thermafil, GuttaCore or vertically compacted gutta-percha from curved root canals assessed by micro-CT. Int Endod J $\underline{51}$, 808815

Ruddle CJ: Cleaning and shaping the root canal system; In: Cohen S, Burns R (Hrsg.): Pathways of the Pulp. 8th edn. St Louis, MO: Mosby 2002, 231-292

Ruddle CJ (2012): Canal preparation: single-file shaping technique. Dent Today 31, 124, 126-129 
Ruiz-Hubard EE, Gutmann JL, Wagner MJ (1987): A quantitative assessment of canal debris forced periapically during root canal instrumentation using two different techniques. J Endod $\underline{13}, 554-558$

Saber SE, Nagy MM, Schäfer E (2015): Comparative evaluation of the shaping ability of ProTaper Next, iRaCe and Hyflex CM rotary NiTi files in severely curved root canals. Int Endod J 쏘, 131-136

Saleh AM, Gilani VP, Tavanafar S, Schäfer E (2015): Shaping ability of 4 different single-file systems in simulated s-shaped canals. J Endod $\underline{41}, 548-552$

Schäfer E, Diez C, Hoppe W, Tepel J (2002): Roentgenographic investigation of frequency and degree of canal curvatures in human permanent teeth. $\mathrm{J}$ Endod $\underline{28}, 211-216$

Schäfer E, Schulz-Bongert U, Tulus G (2004): Comparison of hand stainless steel and nickel titanium rotary instrumentation: a clinical study. J Endod $\underline{30}$, 432-435

Schäfer E, Zapke K (2000): Effizienz maschineller Wurzelkanalaufbereitungssysteme im Vergleich zur manuellen Instrumentierung. Quintessenz 51, 115-124

Schilder H (1974): Cleaning and shaping the root canal. Dent Clin North Am $\underline{18}$, 269-296

Schneider SW (1971): A comparison of canal preparations in straight and curved root canals. Oral Surg Oral Med Oral Pathol $\underline{32}$, 271-275

Schulze RKW, Berndt D, d'Hoedt B (2010): On cone-beam computed tomography artifacts induced by titanium implants. Clin Oral Implants Res $\underline{21}$, 100-107

Sendoline (2018a): 1-Feilen-System für moderne Endodontie http://www.sendoline.com/wp-content/uploads/2016/08/S1-System-BroschyrDE_REV00_low1.pdf; abgerufen am 17.04.2018

Sendoline $\quad$ (2018b): $\quad$ S1 Winkelstück Broschüre http://www.sendoline.com/de/products/treatment/equipment/s1-contra-anglehandpiece/; abgerufen am 17.04.2018 
Shen Y, Gao Y, Lin J, Ma J, Wang Z, Haapasalo M (2013): Methods and models to study irrigation. Endod Topics $\underline{27}$, 3-34

Silva EJNL, Pacheco PT, Pires F, Belladonna FG, De-Deus G (2017): Microcomputed tomographic evaluation of canal transportation and centring ability of ProTaper Next and Twisted File Adaptive systems. Int Endod J $\underline{50}$, 694699

Siqueira JF, Jr., Alves FRF, Versiani MA, Rôças IN, Almeida BM, Neves MAS, Sousa-Neto MD (2013): Correlative bacteriologic and micro-computed tomographic analysis of mandibular molar mesial canals prepared by selfadjusting file, reciproc, and twisted file systems. J Endod $\underline{39}$, 1044-1050

Siqueira JF, Jr., Lima KC, Magalhães FA, Lopes HP, de Uzeda M (1999): Mechanical reduction of the bacterial population in the root canal by three instrumentation techniques. J Endod 25, 332-335

Siqueira JF, Jr., Rôças IN, Lopes HP (2002): Patterns of microbial colonization in primary root canal infections. Oral Surg Oral Med Oral Pathol Oral Radiol Endod $\underline{93}, 174-178$

Skidmore AE, Bjorndal AM (1971): Root canal morphology of the human mandibular first molar. Oral Surg Oral Med Oral Pathol $\underline{32}, 778-784$

Smith CS, Setchell DJ, Harty FJ (1993): Factors influencing the success of conventional root canal therapy - a five-year retrospective study. Int Endod J $\underline{26}$, 321-333

Spili $\mathrm{P}$, Parashos $\mathrm{P}$, Messer HH (2005): The impact of instrument fracture on outcome of endodontic treatment. J Endod $\underline{31}, 845-850$

Stringheta CP, Bueno CEDS, Kato AS, Freire LG, Iglecias EF, Santos M, Pelegrine RA (2019): Micro-computed tomographic evaluation of the shaping ability of four instrumentation systems in curved root canals. Int Endod J $\underline{52}$, 908916

Tachibana H, Matsumoto K (1990): Applicability of X-ray computerized tomography in endodontics. Endod Dent Traumatol $\underline{6}, 16-20$ 
Thomas JP, Lynch M, Paurazas S, Askar M (2020): Micro-computed tomographic evaluation of the shaping ability of WaveOne Gold, TRUShape, EdgeCoil, and XP-3D Shaper endodontic files in single, oval-shaped canals: an in vitro study. J Endod 느, 244-251.e241

Thompson SA (2000): An overview of nickel-titanium alloys used in dentistry. Int Endod J $\underline{33}, 297-310$

Topçuoglu HS, Duzgun S, Akti A, Topçuoglu G (2016): In vitro comparison of cyclic fatigue resistance of ProTaper Next, HyFlex CM, OneShape, and ProTaper Universal instruments in a canal with a double curvature. J Endod 42, 969-971

Topçuoglu HS, Duzgun S, Akti A, Topçuoglu G (2017): Laboratory comparison of cyclic fatigue resistance of WaveOne Gold, Reciproc and WaveOne files in canals with a double curvature. Int Endod J $\underline{50}, 713-717$

Tzanetakis GN, Kontakiotis EG, Maurikou DV, Marzelou MP (2008): Prevalence and management of instrument fracture in the postgraduate endodontic program at the Dental School of Athens: a five-year retrospective clinical study. J Endod $\underline{34}, 675-678$

Ungerechts C, Bårdsen A, Fristad I (2014): Instrument fracture in root canals where, why, when and what? A study from a student clinic. Int Endod J $\underline{47}$, 183190

Uslu G, Özyürek T, Yilmaz K, Gündogar M (2018): Cyclic fatigue resistance of RPilot, HyFlex EDM and PathFile nickel-titanium glide path files in artificial canals with double (S-shaped) curvature. Int Endod J $\underline{51}$, 584-589

van der Vyver PJ, Paleker F, Vorster M, de Wet FA (2019): Root canal shaping using nickel titanium, M-Wire, and Gold Wire: a micro-computed tomographic comparative study of One Shape, ProTaper Next, and WaveOne Gold instruments in maxillary first molars. J Endod $\underline{45}, 62-67$

VDW (2018): Die Wahl des passenden RECIPROC Instruments https://www.vdw-dental.com/de/sortiment/produktdetail/reciproc-feilen/; abgerufen am 12.04.2018

Versiani MA, Carvalho KKT, Mazzi-Chaves JF, Sousa-Neto MD (2018): Microcomputed tomographic evaluation of the shaping ability of XP-endo Shaper, $\mathrm{iRaCe}$, and EdgeFile Systems in long oval-shaped canals. J Endod 44, 489-495 
Versiani MA, Pécora JD, Sousa-Neto MD (2013): Microcomputed tomography analysis of the root canal morphology of single-rooted mandibular canines. Int Endod J $\underline{46}, 800-807$

Vertucci FJ (2005): Root canal morphology and its relationship to endodontic procedures. Endod Topics 10, 3-29

Viana ACD, de Melo MCC, de Azevedo Bahia MG, Buono VTL (2010): Relationship between exibility and physical, chemical, and geometric characteristics of rotary nickel-titanium instruments. Oral Surg Oral Med Oral Pathol Oral Radiol Endod 110, 527-533

Walia HM, Brantley WA, Gerstein H (1988): An initial investigation of the bending and torsional properties of Nitinol root canal files. J Endod 14, 346-351

Weine FS, Kelly RF, Bray KE (1976): Effect of preparation with endodontic handpieces on original canal shape. J Endod 2, 298-303

Weine FS, Kelly RF, Lio PJ (1975): The effect of preparation procedures on original canal shape and on apical foramen shape. J Endod 1, 255-262

Withers PJ (2007): X-ray nanotomography. Mater Today $\underline{10}$, 26-34

Wu MK, R'oris A, Barkis D, Wesselink PR (2000): Prevalence and extent of long oval canals in the apical third. Oral Surg Oral Med Oral Pathol Oral Radiol Endod $\underline{89}, 739-743$

Yamamura B, Cox TC, Heddaya B, Flake NM, Johnson JD, Paranjpe A (2012): Comparing canal transportation and centering ability of endosequence and vortex rotary files by using micro-computed tomography. J Endod 38, 1121-1125

Yang G, Yuan G, Yun X, Zhou X, Liu B, Wu H (2011): Effects of two nickeltitanium instrument systems, Mtwo versus ProTaper universal, on root canal geometry assessed by micro-computed tomography. J Endod 37, 1412-1416

Yared G (2008): Canal preparation using only one $\mathrm{Ni}-\mathrm{Ti}$ rotary instrument: preliminary observations. Int Endod J 41, 339-344

Yin X, Cheung GS, Zhang C, Masuda YM, Kimura Y, Matsumoto K (2010): Microcomputed tomographic comparison of nickel-titanium rotary versus traditional instruments in C-shaped root canal system. J Endod $\underline{36}$, 708-712 
You SY, Kim HC, Bae KS, Baek SH, Kum KY, Lee W (2011): Shaping ability of reciprocating motion in curved root canals: a comparative study with microcomputed tomography. J Endod $\underline{37}, 1296-1300$

Yousuf MA, Asaduzzaman M (2010): An efficient ring artifact reduction method based on projection data for micro-CT images. J Sci Res $\underline{2}, 37-45$

Zandbiglari T, Davids H, Schäfer E (2006): Influence of instrument taper on the resistance to fracture of endodontically treated roots. Oral Surg Oral Med Oral Pathol Oral Radiol Endod 101, 126-131

Zanette F, Grazziotin-Soares R, Flores ME, Fontanella VRC, Gavini G, Barletta FB (2014): Apical root canal transportation and remaining dentin thickness associated with ProTaper Universal with and without PathFile. J Endod $\underline{40}$, 688693

Zhou H, Peng B, Zheng Y (2013): An overview of the mechanical properties of nickel-titanium endodontic instruments. Endod Topics $\underline{29}, 42-54$

Zinelis S, Eliades T, Eliades G (2010): A metallurgical characterization of ten endodontic $\mathrm{Ni}-\mathrm{Ti}$ instruments: assessing the clinical relevance of shape memory and superelastic properties of $\mathrm{Ni}$-Ti endodontic instruments. Int Endod J $\underline{43}, 125-$ 134

Zupanc J, Schäfer E (2017): Neue Nickel-Titan-Legierungen. Endodontie 26, 269-281 